\title{
Gas Turbine Engine Performance Estimation and Prediction
}

\author{
by \\ Houman Hanachi
}

A thesis submitted to the Faculty of Graduate and Postdoctoral Affairs in partial fulfillment of the requirements for the degree of

Doctor of Philosophy

in

Mechanical Engineering

Carleton University

Ottawa, Ontario

(C) 2015, Houman Hanachi 


\section{Abstract}

Modern health management approaches for gas turbine engines (GTE) aim to acquire precise information about the health state of the GTE components to optimize the maintenance decisions with respect to both the economy and safety. The task becomes more challenging for the GTE parts inaccessible to direct measurements with the available sensors of the GTE control system. This article-based thesis integrates a set of five coherent research work to address this problem.

A detailed nonlinear thermodynamic model for single shaft GTEs is developed to predict the expected cycle parameters for the GTE in the healthy condition. In reality, the measured cycle parameters gradually deviate from the prediction due to performance deterioration. Physics-based performance indicators are defined based on the deviations in the measured performance parameters, compared to the respective model predictions. The indicators can effectively monitor the GTE performance deterioration in both shortterm and long-term regimes. In the next step, effect of the air humidity is taken into account to enhance the GTE model, and it is shown that the enhanced model can improve the performance monitoring by reducing the uncertainties.

In order to separate the effects of different fault modes, an inference-based model is developed to predict the short-term recoverable performance deterioration due to the compressor fouling under different ambient and operating conditions. For the long-term non-recoverable performance deterioration due to the degradation mechanisms in the turbine hot section, two steps are undertaken; 1) a state estimation framework is developed for nonlinear/non-Gaussian systems with non-uniform time steps to track a degradation symptom of the turbine, i.e., loss of isentropic efficiency, using the observable performance indicators, and 2) the state estimation framework is extended to multidimensional dynamical systems with stochastic inputs for simultaneous tracking of two degradation symptoms, i.e., loss of isentropic efficiency and increase of the mass flow, using the observable parameters, provided by the GTE operating system.

The developed techniques and frameworks are verified and validated, using a set of three-year operating data from an industrial GTE in a power plant. 


\section{Preface}

This thesis is an integrated article-based thesis, consisted of the peer-reviewed journal articles and a conference paper, either published or currently under review.

In all of the work presented henceforth, Houman Hanachi was the principal contributor in all areas of conceptualization, methodology development, implementation and validation, computer code writing, data preprocessing and analysis, as well as manuscript composition, including tables and figures. Professor Jie Liu provided the guidance and supervision of the overall project as well as timely and constructive feedbacks to all the research work. Dr. Avisekh Banerjee provided his ideas and guidance regarding the work as well as editorial suggestions. Dr. Ying Chen provided supportive explanations on the details of gas turbine mechanism including design-point parametric values and the performance maps for off-design modeling. He also provided his ideas and guidance throughout the research. Dr. Ashok Koul provided his overall ideas, the guidelines and advices for the corresponding co-authored article.

A version of Chapter 2 has been published in IEEE Transactions on Reliability, [H. Hanachi, J. Liu, A. Banerjee, Y. Chen and A. Koul, "A Physics-based modeling approach for performance monitoring in gas turbine engine," IEEE Transactions on Reliability, vol. 64, pp. 197-205, 2014].

A version of Chapter 3 has been presented in ASME Turbo Expo 2015, Montreal, Canada, [H. Hanachi, J. Liu, A. Banerjee and Y. Chen, "Effects of the intake air humidity on the gas turbine performance monitoring," in ASME Turbo Expo 2015, Montreal, Canada, 2015].

A version of Chapter 4 has been published in Measurement Science and Technology [H. Hanachi, J. Liu, A. Banerjee and Y. Chen, "A framework with nonlinear system model and nonparametric noise for gas turbine degradation state estimation," Measurement Science and Technology, vol. 26, 065604, 2015].

A version of Chapter 5 has been published in Journal of Engineering for Gas Turbines and Power, [H. Hanachi, J. Liu, A. Banerjee and Y. Chen, " Effects of humidity 
condensation on the trend of gas turbine performance deterioration," ASME Journal of Engineering for Gas Turbines and Power, vol. 137(12), pp. 122605-11, 2015].

A version of Chapter 6 has been submitted to Mechanical Systems and Signal Processing and is currently under review.

Modifications have been made to the articles to meet the requirements of the integrated-thesis format and to maintain consistency of the thesis. In particular, introduction and conclusion sections of the papers have been shortened and revised, symbols of some parameters have been renamed to maintain uniformity of the symbols throughout the chapters of the thesis, a single list for the symbols has been included before Chapter 1, and a single list of references has been added at the end.

Permissions to use the published articles as chapters in this thesis have been obtained from the publishers, as indicated by the letters included along with the thesis manuscript. Readers who wish to cite the corresponding material should cite the original papers as per the citations mentioned above. 


\section{Acknowledgements}

I would like to gratefully acknowledge the encouragement, advice and critique that my supervisor Professor Jie Liu has provided to me during the course of my PhD program. This work could not have been accomplished without the sincere support he provided with great patience.

I appreciate the support provided by the industry partner of this research, Life Prediction Technologies Inc., and the excellent people, Dr. Ashok Koul, Dr. Avisekh Banerjee and Dr. Ying Chen, for their great helps and guidance.

I thank my parents and siblings, and my wife's family for all their encouragement and love. I thank my son Mohammad Ali for forgiving me for being away so much, and most of all, I thank my wife Marjan for her understanding and all she has sacrificed to support me through my study. 


\section{Table of Contents}

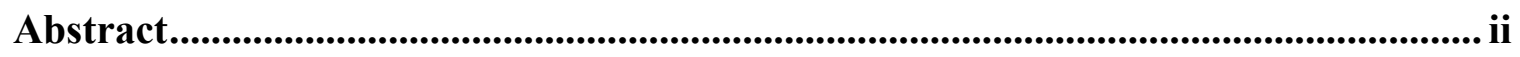

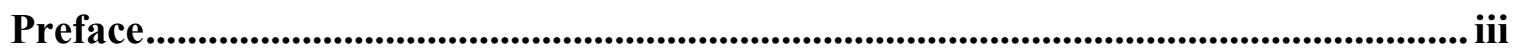

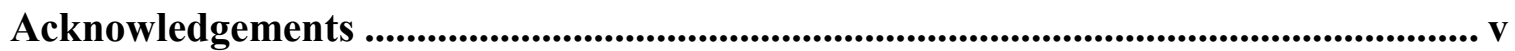

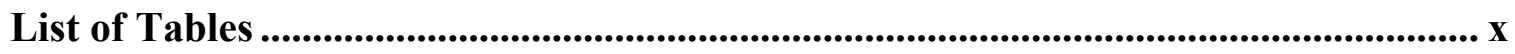

List of Figures............................................................................................................................... xi

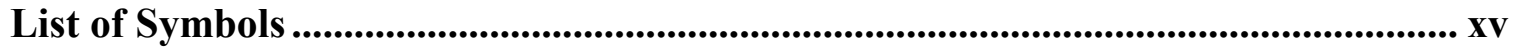

Chapter 1: Introduction ....................................................................................................... 1

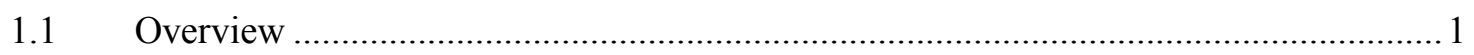

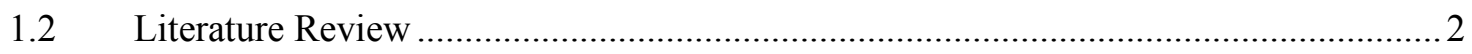

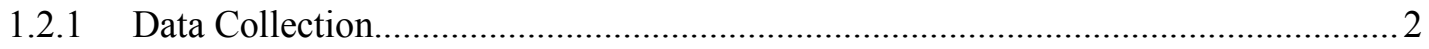

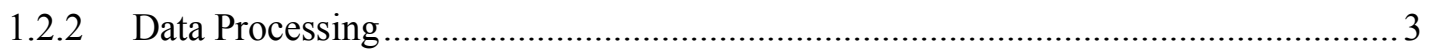

1.2.3 GTE Fault Detection and Diagnostics............................................................. 4

1.2.4 Degradation State Estimation and Prognostics ................................................. 7

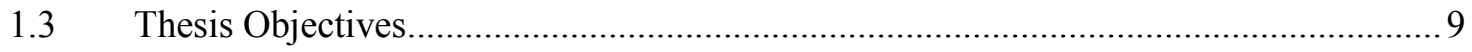

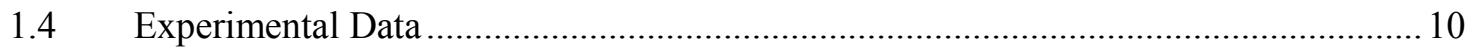

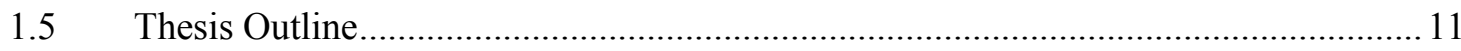

\section{Chapter 2: A Physics-Based Modeling Approach for Performance Monitoring in}

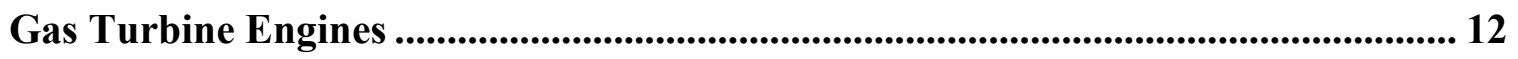

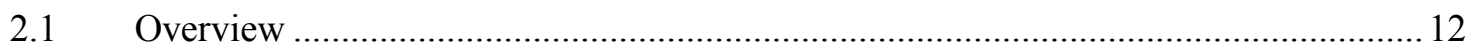

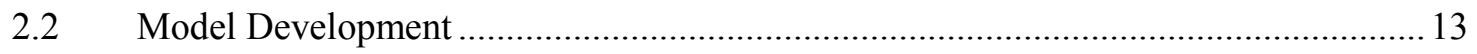

2.2.1 The Thermodynamic Model for the Single Shaft GTE ......................................... 13

2.2.2 Performance Indicators from the Over-Determined Model .................................... 16

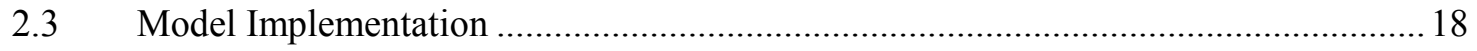




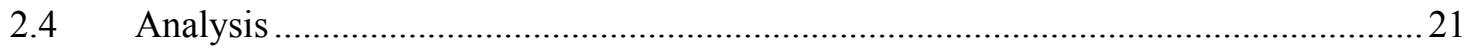

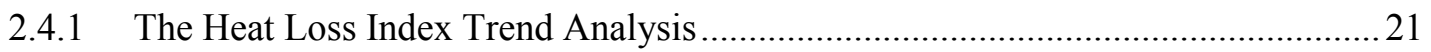

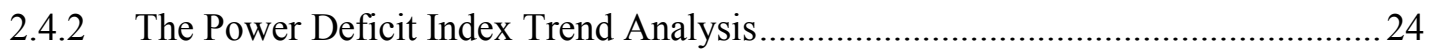

2.4.3 A comparative Study on the Performance Indicators............................................27

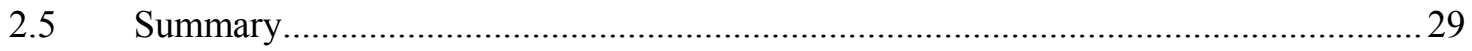

\section{Chapter 3: Effects of Intake Air Humidity on the Gas Turbine Performance}

Monitoring ...................................................................................................................................... 30

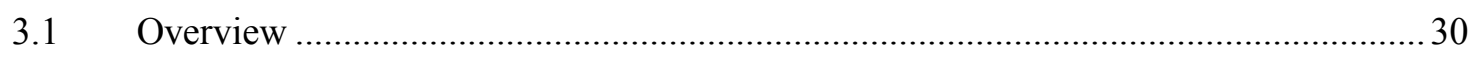

3.2 Gas Turbine Model with Humid Air ........................................................................ 30

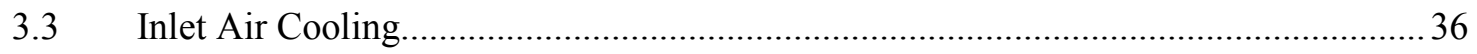

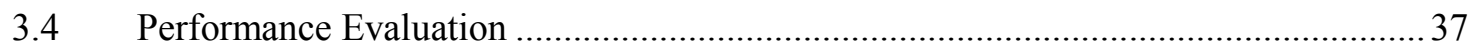

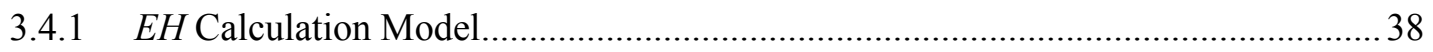

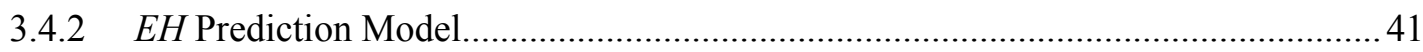

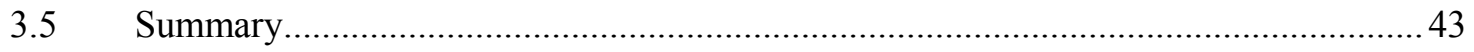

\section{Chapter 4: A Framework with Nonlinear System Model and Nonparametric Noise} for Gas Turbine Degradation State Estimation .............................................................. 44

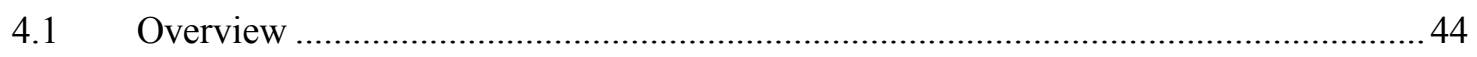

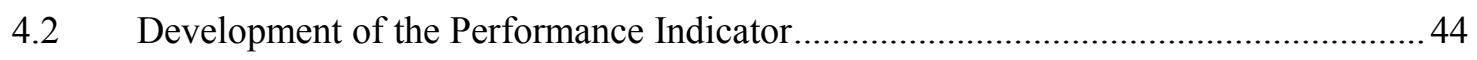

4.2.1 Thermodynamic Model of the GTE for Performance Monitoring .......................... 44

4.2.2 Turbine Degradation Symptom: the Internal State ............................................... 48

4.3 Sequential State Estimation for Nonlinear Systems with Non-Gaussian Noise ...........51

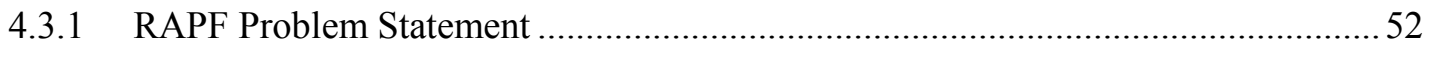

4.3.2 RAPF in Non-Uniform Time Steps and Redundant Measurements .......................56

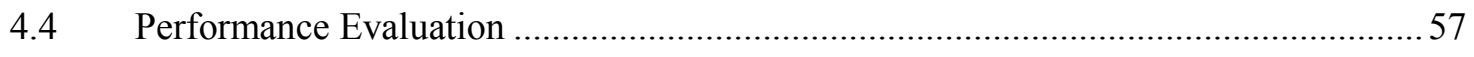

4.4.1 Benchmark Model with Non-Uniform Steps and Redundant Measurements ...........58

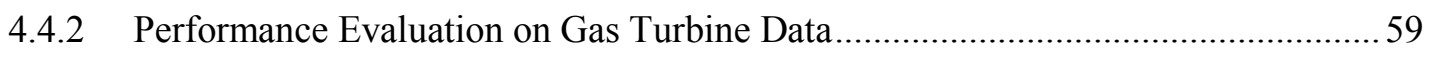




\section{Chapter 5: Effects of Humidity Condensation on the Trend of Gas Turbine}

Performance Deterioration ................................................................................. 64

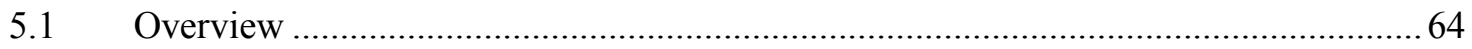

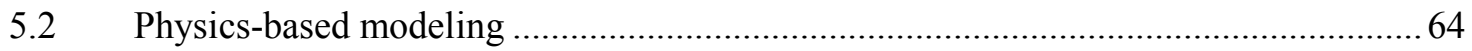

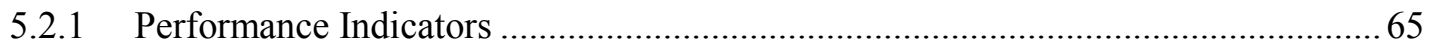

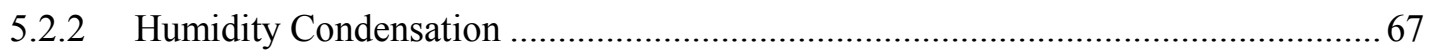

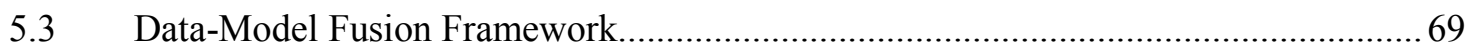

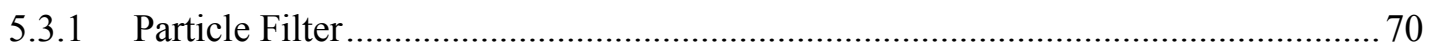

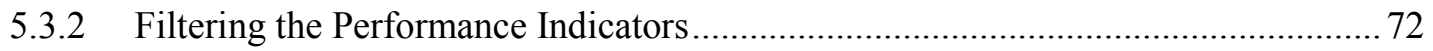

5.4 Effective Factors on the Rate of Performance Deterioration......................................73

5.4.1 Effects of Power and Condensed Humidity ......................................................... 74

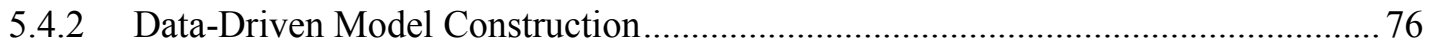

5.5 Performance Deterioration Modeling Using ANFIS ….......................................... 76

5.5.1 Building ANFIS Structure and Training ........................................................... 77

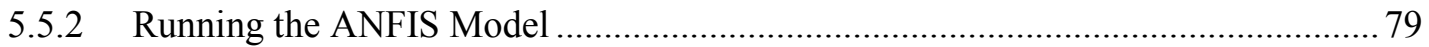

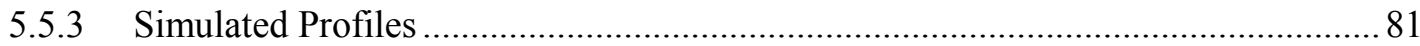

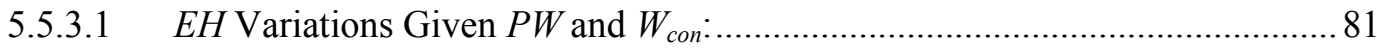

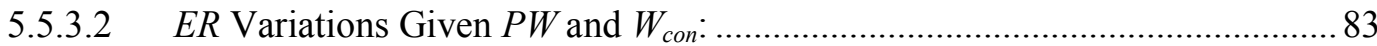

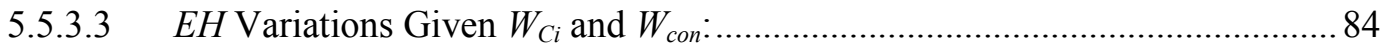

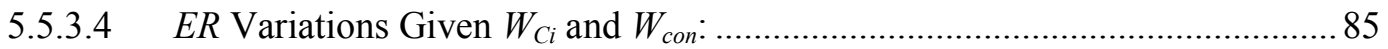

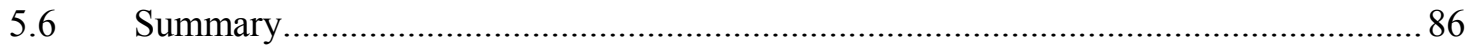

\section{Chapter 6: Sequential State Estimation of Nonlinear/Non-Gaussian Systems with} Stochastic Input for Turbine Degradation Monitoring.................................................. 87

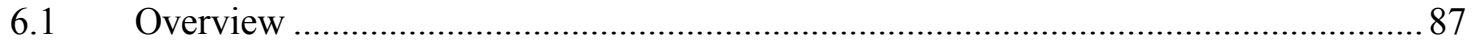

6.2 Sequential State Estimation for Nonlinear System with Stochastic Input (NSI)..........88 


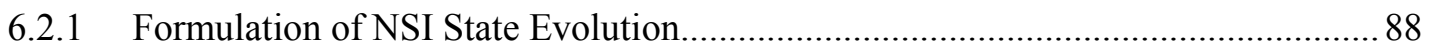

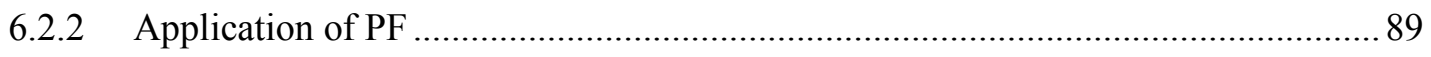

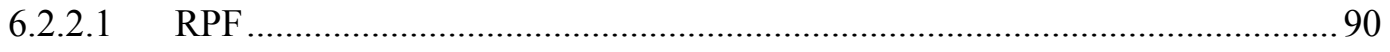

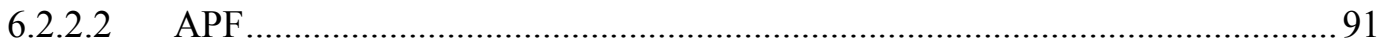

6.2.3 Performance Evaluation on Benchmark Model …................................................ 93

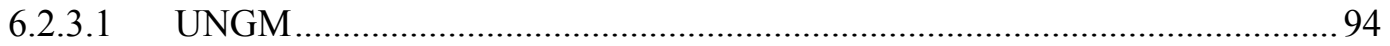

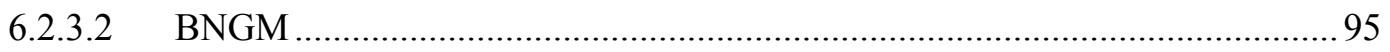

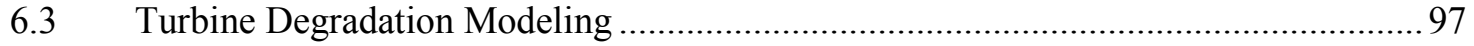

6.3.1 Gas-path Model of the Degrading Turbine ........................................................ 98

6.3.2 Turbine Degradation Prediction Model............................................................... 102

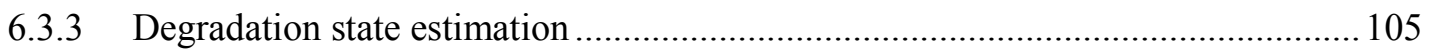

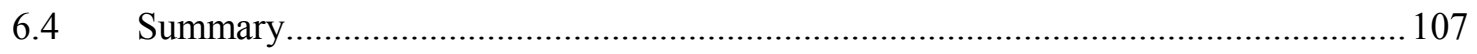

Chapter 7: Contributions, Conclusions and Recommendations ........................... 109

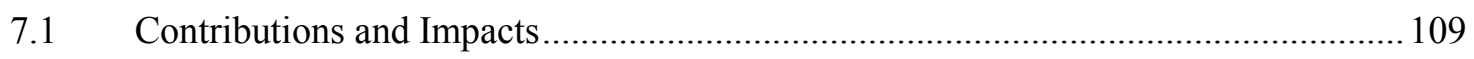

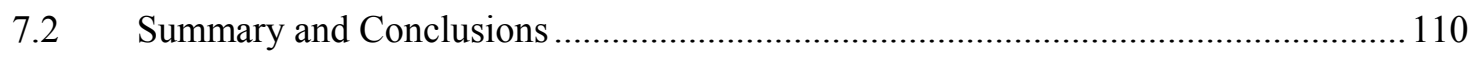

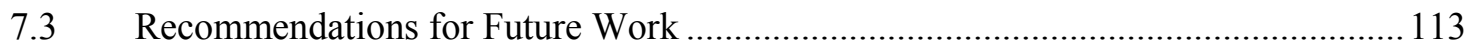

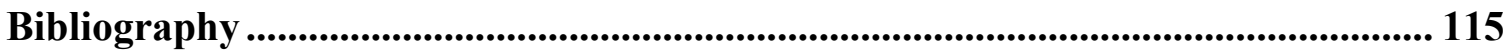

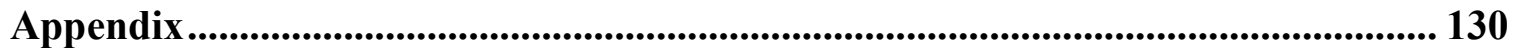




\section{List of Tables}

Table 1.1. Basic technical specifications of the GTE at the design point....................... 10

Table 1.2. Available ambient and operating parameters........................................... 11

Table 2.1. Sample data from the compressor map ..................................................... 19

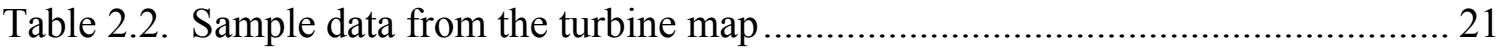

Table 2.3. Variation rate of the performance indicators in selected periods .................. 26

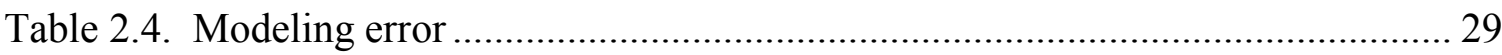

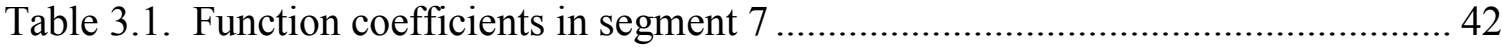

Table 4.1. Efficiency loss and modeling error .......................................................... 51

Table 4.2. RAPF technique with rejection of low likelihood corresponding particles .... 55

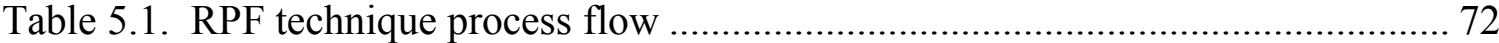

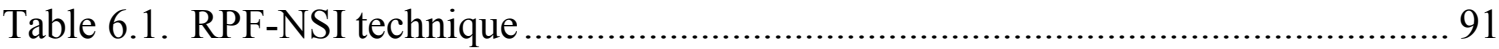

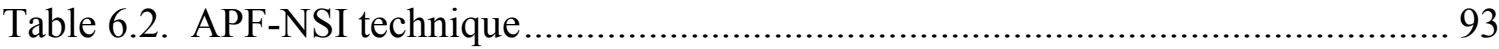

Table 6.3. State estimation errors averaged over 100 simulations ................................ 97

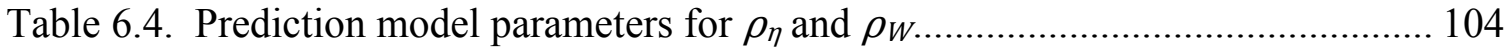




\section{List of Figures}

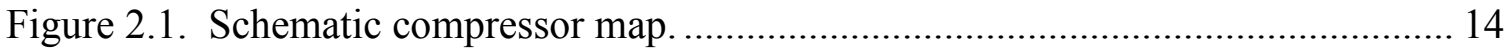

Figure 2.2. The model calculates both the heat loss index and the power deficit index.. 20 Figure 2.3. Variation of $E G T$ throughout the operating time: (a) the normalized measured $E G T$, and (b) the normalized expected $E G T$

Figure 2.4. The heat loss index shows distinct short-term and long-term variation trends:

(a) the original signal, and (b) the filtered signal. 23

Figure 2.5. Variation of the GTE output power: (a) the normalized measured power, and

(b) the normalized expected power. 25

Figure 2.6. The short-term, and the long-term variation trends are observable in the power deficit index: (a) the original signal, and (b) the filtered signal......................... 26

Figure 2.7. The short-term variation rate of the performance indicators fluctuate

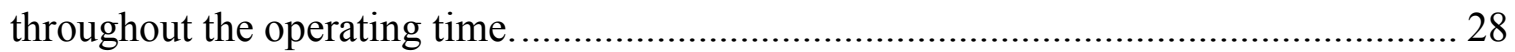

Figure 3.1. Schematic turbine performance map................................................. 33

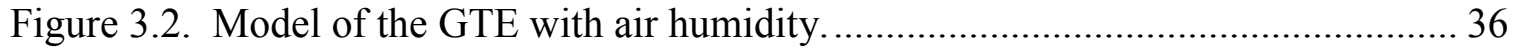

Figure 3.3. Variations of the ambient condition and the moisture content: (a) ambient temperature, (b) ambient relative humidity, and (c) specific humidity at the GTE inlet.. 40 Figure 3.4. Variations of the performance indicator: (a) $E H_{a}$, (b) $E H_{h}$, and (c) the difference: $\Delta E H$.

Figure 3.5. Variations of $\Delta E H_{n}$ statistical characteristics: (a) standard deviation, and (b)

mean value. 41

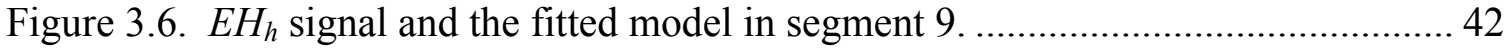


Figure 3.7. Standard deviation ratio for the modeling errors.

Figure 4.1. Process flow to calculate $E R$ indicator. 46

Figure 4.2. Performance deterioration due to the short-term and the long-term degradations: (a) overall performance variation, and (b) Selected segments depicting short-term performance variation. 48

Figure 4.3. Forward causality and backward inference in the GTE degradation. 49 Figure 4.4. (a) Long-term performance deterioration: decline of $E R$ index, and (b) degradation of the turbine: ratio of the isentropic efficiency loss. 50 Figure 4.5. Performance of the state estimation techniques: (a) RAPF technique, (b) RPF technique. 59

Figure 4.6. Degradation model and modeling uncertainty pdf. 60 Figure 4.7. Measurement likelihood empirical density in different steps 61

Figure 4.8. Distribution of the state particles and corresponding weights 61 Figure 4.9. Comparative results :(a) RAPF trajectories with 200 particles (green) and the expected trajectory (blue), compared with the expected RPF trajectory (red), and (b) Comparison of repeatability: standard deviation for 20 runs. 62 Figure 5.1. Performance deterioration during the operating life. (a) EH performance indicator, and (b) ER performance indicator. 66 Figure 5.2. Humidity in inlet air: (a) total humidity, (b) condensed humidity, and (c) comparison in elected time window.

Figure 5.3. RPF state estimation (red) on the performance indicator signals (blue): (a) $E H$ performance indicator, and (b) $E R$ performance indicator. 
Figure 5.4. Variation rates of the performance indicators do not constitute functions over $P W-W_{c o n}$ space: (a) variation rate of $E H$, and (b) variation rate of $E R$. 75

Figure 5.5. ANFIS structure for system identification. Dashed line shows the use of output as input of the next step for profile simulation. 78

Figure 5.6. Training data points and the result of ANFIS model prediction: (a) $E H$ data and prediction, (b) ER data and prediction, and (c) scarcity of data points in some regions of the domain. 80

Figure 5.7. ANFIS model simulated profiles for $E H$ : (a) $E H$ profiles at constant power and humidity condensation rates, and (b) $E H$ profiles at $P W=2400 \mathrm{~kW}$. 82

Figure 5.8. ER simulated profiles at $P W=3500 \mathrm{~kW}$ 84

Figure 5.9. $E H$ simulated profiles at $W_{C i}=16.75 \mathrm{~kg} / \mathrm{s}$. 85

Figure 5.10. Lack of training data points for $E R$ simulation with $W_{C i}-W_{c o n}$ data............ 85

Figure 6.1. (a) System input, and (b) Observed output........................................... 95

Figure 6.2. NSI state estimation by RPF and APF. 95

Figure 6.3. System input and output: (a) Input real part, (b) Input imaginary part, (c)

Observed output real part, and (d) Observed output imaginary part. 96

Figure 6.4. Bivariate NSI state estimation (a) RPF real part, (b) RPF imaginary part, (c) APF real part, and (d) APF imaginary part. 97 Figure 6.5. Variation of typical GPP vs efficiency decrease $\rho_{\eta}$ and mass flow increase $\rho_{W}$. Operating parameters are fixed at $T_{C i}=15^{\circ} \mathrm{C}, P_{C i}=101 \mathrm{kPa}, \phi_{C i}=50 \%, N=16500 \mathrm{RPM}$, $P W=4 \mathrm{MW}$. 99 Figure 6.6. Measured operating parameters of the GTE: (a) Power, (b) EGT, and (c) fuel pressure. 101 
Figure 6.7. Variation of the turbine degradation symptoms during the operation, and corresponding prediction models: (a) $\rho_{\eta}$ symptom, and (b) $\rho_{W}$ symptom. 101 Figure 6.8. Degradation symptoms, sampled at time-step 1, 10 and 15; (a) distribution of $\left[\rho_{\eta}, \rho_{W}\right]$ in each sampling time-step, (b) Joint pdf of $\left[\rho_{\eta}, \rho_{W}\right]$ at each sampling time-step.

Figure 6.9. Empirical joint distribution of the modeling error. 105

Figure 6.10. State estimation results by RPF (blue) and APF (red) using 100 particles: (a) $\rho_{\eta}$ symptom, and (b) $\rho_{W}$ symptom. 106 


\section{List of Symbols}

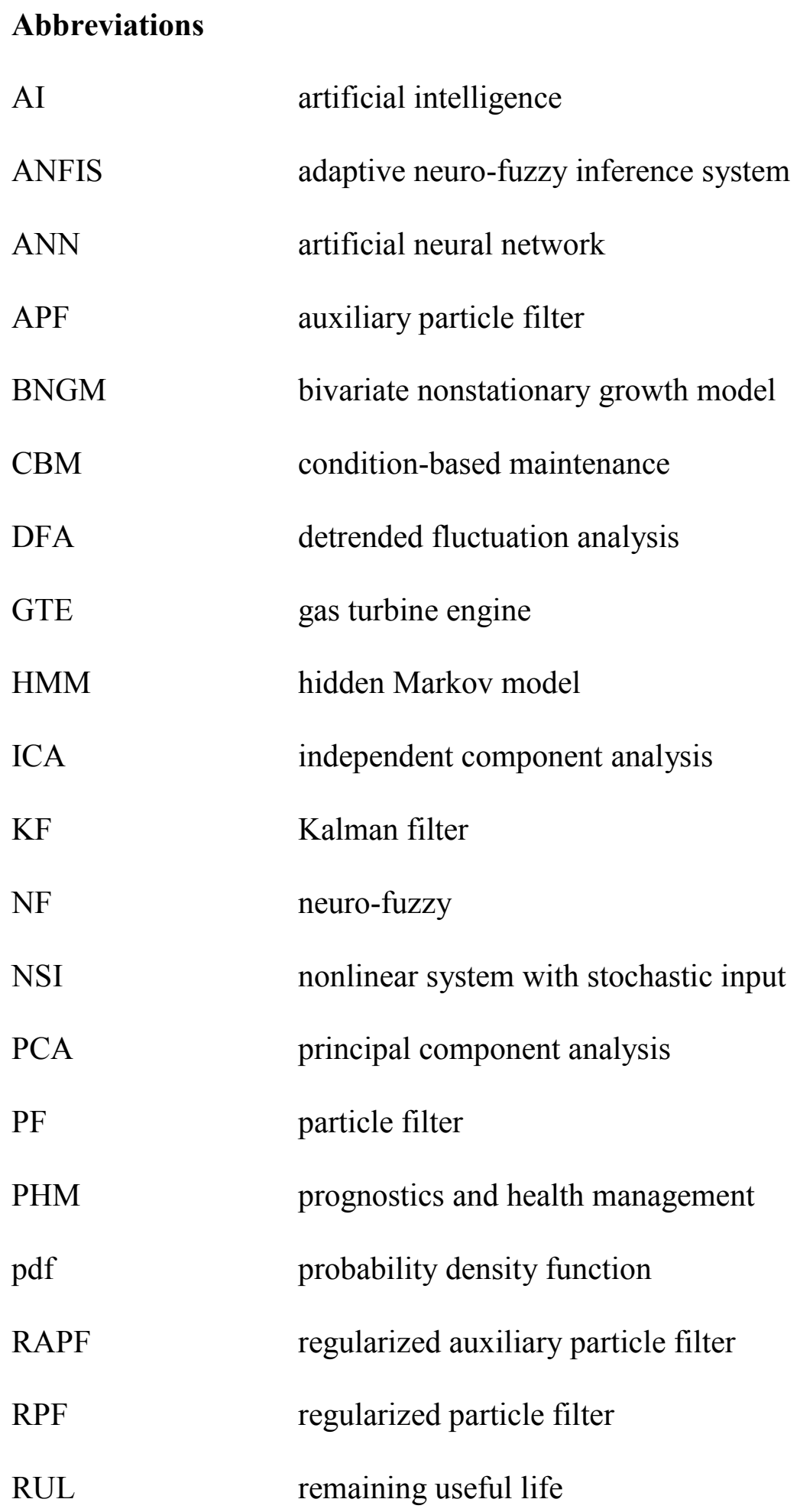


SLAM

TBO

UNGM

\section{Notation}

A

$c_{p}$

D

$D P$

$E[$.

EGT

EH

ER

$F($.

$F($.

$f$

$f($.

$G($.

GPP

$g($.

H

$h$

h simultaneous localization and mapping

time between overhauls

univariate nonstationary growth model

cross-sectional area

specific heat capacity $(\mathrm{kJ} / \mathrm{kg} \mathrm{K})$

dimension of state vector

design parameters

expected value

exhaust gas temperature (K)

excess heat ratio

efficiency ratio

state model

mathematical function

fuel to air mass ratio

probability density

measurement model

gas-path parameters

importance density

enthalpy $(\mathrm{kJ})$

specific enthalpy $(\mathrm{kJ} / \mathrm{kg})$

kernel bandwidth 


\begin{tabular}{|c|c|}
\hline$I_{H L}$ & heat loss index \\
\hline$I_{P D}$ & power deficit index \\
\hline$i$ & particle index \\
\hline$j$ & index of sampled particle \\
\hline$K()$. & kernel density \\
\hline$k$ & time-step index \\
\hline$L$ & state model constant parameter \\
\hline$L C V$ & lower calorific value $(\mathrm{kJ} / \mathrm{kg})$ \\
\hline Map & mathematical function for performance map \\
\hline$m$ & number of particles \\
\hline$N$ & shaft rotational speed (RPM) \\
\hline$n$ & number of time-steps \\
\hline$P$ & total pressure $(\mathrm{kPa})$ \\
\hline$P_{w}^{*}$ & saturation vapor pressure $(\mathrm{Pa})$ \\
\hline$P R$ & pressure ratio \\
\hline$P W$ & power $(\mathrm{kW})$ \\
\hline$R$ & specific gas constant $(\mathrm{kJ} / \mathrm{kg} \mathrm{K})$ \\
\hline SH & specific humidity \\
\hline$S T D$ & standard deviation \\
\hline$s$ & number of measurement samples \\
\hline$T$ & total temperature $(\mathrm{K})$ \\
\hline$t$ & time (hour) \\
\hline$U$ & input history \\
\hline
\end{tabular}




\begin{tabular}{|c|c|}
\hline$u$ & system input \\
\hline$V$ & velocity $(\mathrm{m} / \mathrm{s})$ \\
\hline$V A$ & fuel valve angle \\
\hline$v$ & specific volume $\left(\mathrm{m}^{3} / \mathrm{kg}\right)$ \\
\hline$W$ & mass flow $(\mathrm{kg} / \mathrm{s})$ \\
\hline$w$ & probability weight threshold \\
\hline$x$ & state \\
\hline$Y$ & output history \\
\hline$y$ & system output \\
\hline$\alpha$ & state model coefficients \\
\hline$\beta$ & auxiliary coordinate in performance map \\
\hline$\gamma$ & specific heat ratio \\
\hline$\varepsilon$ & state model error \\
\hline$\Delta t$ & time length between the steps (hour) \\
\hline$\delta()$. & Dirac delta function \\
\hline$\eta$ & efficiency \\
\hline$\theta_{c_{p}}$ & correction parameter for $c_{p}$ of product $(\mathrm{kJ} / \mathrm{kg} \mathrm{K})$ \\
\hline$\kappa$ & input noise \\
\hline$\lambda$ & state model constant parameter \\
\hline$\mu$ & representative state particle \\
\hline$v$ & measurement noise \\
\hline$\rho$ & degradation symptom \\
\hline$\tau$ & process noise \\
\hline
\end{tabular}




$\begin{array}{ll}\phi & \text { relative humidity } \\ \psi & \text { logistic function of power } \\ \omega & \text { probability weights }\end{array}$

\section{Subscripts and Accents}

$\begin{array}{ll}A & \text { actual value } \\ a & \text { air } \\ \text { am } & \text { ambient } \\ b & \text { bleed } \\ C & \text { compressor } \\ \text { Ch } & \text { combustion chamber } \\ \text { con } & \text { condensed humidity } \\ \text { cor } & \text { corrected value } \\ \text { D } & \text { design point } \\ \text { exh } & \text { exhaust } \\ \text { exp } & \text { expected } \\ F & \text { fuel } \\ \text { Fil } & \text { filter } \\ h & \text { humid air } \\ I & \text { imaginary part } \\ \text { i } & \text { inlet } \\ L & \text { loss } \\ M & \text { modeled quantity }\end{array}$




$\begin{array}{ll}\text { Mix } & \text { mixture } \\ o & \text { outlet } \\ P & \text { combustion product } \\ R & \text { real part } \\ S & \text { static } \\ \text { std } & \text { standard atmosphere } \\ T & \text { turbine } \\ w & \text { water (liquid or vapor) } \\ - & \text { mean } \\ \hat{.} & \text { estimated by regression }\end{array}$




\section{Chapter 1: Introduction}

\subsection{Overview}

Gas turbine engines (GTE) are widely used for power generation, ranging from stationary power plants to airplane propulsion systems. For GTE operating industries, availability, reliability and maintenance costs are of the main concerns, and maintenance strategies have constantly progressed to address such concerns. In conventional strategy, maintenance services are provided based on a pre-scheduled plan regardless of the health state of an individual GTE. Consequently, a GTE may be sent for maintenance service when it is still in a healthy condition or, on the other hand, it may fail before the scheduled service is due. GTE availability will drop and maintenance costs will increase with conventional maintenance strategy as a result. Condition-based maintenance (CBM) is an effective strategy to improve engine availability and reduce maintenance costs and failure hazards. In this strategy, GTE health information is collected from diagnostic and prognostic analyses [1]. This is the basic idea of prognostics and health management (PHM) systems, which takes the following steps in its implementation: 1) The GTE is monitored using sensors in critical points of the machine. The sensors measure physical parameters, e.g., temperature, pressure, rotary speed and vibration. 2) The next step is to extract features from the measurements and to identify anomalies among the extracted features. 3) Future health state of the GTE is estimated using the current state of the system, and the remaining useful life (RUL) is predicted [2].

The performance of a GTE gradually deteriorates during the operation. There are two major mechanisms contributing to this phenomenon. A rapid deterioration takes place due to fouling and congestion of the air particulates mostly in the upstream sections of the gas turbine. The performance can be restored if the congested material is removed by washing [3]. At the same time, a slower performance deterioration occurs due to structural degradation of the parts such as erosion and corrosion processes. This cannot be recovered, unless the defective parts are repaired or replaced [4]. Development of an effective PHM system for the GTEs entails a comprehensive knowledge on the trend of performance deterioration, both on the short-term and the long-term regimes, respectively caused by recoverable and non-recoverable degradation mechanisms. It becomes more 
complicated when there is no measurements available from the internal gas path parameters, which is the case for most of the GTEs in service. To address this shortcoming especially for the huge fleet of the conventional GTEs, this thesis is focused on the development of techniques for quantitative performance assessment of the GTEs and distinctive performance prediction in long-term and short-term regimes in subsystem level.

\subsection{Literature Review}

This thesis is an integrated article based work, and the literature review section covers the background literature on the overall field of gas turbine degradation monitoring including: data collection, data processing and performance deterioration modeling. Specific literature review pertaining to the topics of each chapter is provided in the corresponding overview sections.

\subsubsection{Data Collection}

Data collection is the first step required in a PHM system. It is the process of collecting and storing data from the monitored system for the purpose of PHM. There are two main types of data collected for PHM application. The first type is called event data, which is the descriptive information on the pertaining events and the provided services, e.g., installation, overhaul, failures and description of maintenance services. The second type; the condition monitoring data are quantitative records on the system parameters and variables related to the health condition of the machine [5].

As a standard setup, GTEs are equipped with a set of sensors to measure the operating parameters of the machine and transmit the corresponding data to the GTE operating system for monitoring and control purposes. Gas path measurements including rotary speed, intake air temperature and exhaust gas temperature (EGT), as well as vibration amplitude data are examples of measured parameters by the GTE primary sensors [6]. In some modern GTEs, tip clearance of the blades is constantly measured for health monitoring $[7,8]$. Such data are readily available in real-time from the operating system of the GTE. In special cases where particular parameters are required to be measured, external instrumentations are employed for data acquisition. This method usually applies 
for case studies, investigations and R\&D purposes. Exhaust gas composition, debris contents of the lubricant oil and acoustic spectrum signals are examples of measurements by such method $[9,10]$.

Most of the fault modes in degradation of the GTE components manifest their symptoms as changes in the gas path measurements [11]. As a result, development of a PHM system that makes use of gas path data from the GTE operating systems can find broader application, especially for the huge fleet of the GTEs already in service. In this research, the gas path data from the GTE operating system are adopted for further processing.

\subsubsection{Data Processing}

Before further processing, the data should be cleaned at the first step. Data cleaning alleviates the risk of using erroneous data in the analysis and modeling. Different factors including human mistakes and sensor faults can contribute to data errors [5]. An effective strategy to clean erroneous data is sensor validation. In this strategy, the integrity of sensed data is checked before it is passed to the next processing modules. Generic signal processing, neural network and fusion models are utilized for sensor validation to ensure the highest possible sensor fault detection with minimal false alarms [12]. Graphical tools are also useful and effective to find and remove data errors [13]. However, it should be noted that there is no general algorithm to clean the data.

The GTE operating system measurements are usually value type data, which are recorded as time series. Even vibration amplitude, which are usually measured by GTE operating systems, are single RMS values and are not considered waveform type data. In practice, some of the measured variables have no meaningful correlation with the other variables in the study. Sensitivity analysis is an effective process to find and eliminate the variables with no correlations and reduce the dimension of data to the meaningful components [14]. Multivariate analysis techniques such as principal component analysis (PCA) and independent component analysis (ICA), which is an extension of PCA, are useful techniques to handle data with complicated correlation structure [15]. Project pursuit is another dimension reduction technique for systems with large number of variables $[15,16]$. 
The next step is feature extraction, i.e., the procedure of extracting useful information from the remaining variables in raw measurements. There are two main approaches for feature extraction, i.e., the statistical approach and the physics-based approach. In the statistical approach, mathematical and statistical techniques are used to find correlations among the elements of the measurement vector time series. Trend analysis techniques such as regression analysis and time series modeling are commonly used for feature extraction in value type data [17]. Detrended fluctuation analysis (DFA) is another useful technique intended to detect the autocorrelations in noisy and non-stationary time series [18]. Linear, polynomial and logistic regression and ARMA models are other statistical techniques to analyze GTE data for feature extraction [19-22].

In physics-based approaches, functional relations are established between the elements of measurement vectors based on the physics laws. When processing the gas path data, laws of thermodynamics are utilized to construct a physics-based model, also known as heat balance model. Depending on the accessible parameters and available computational resources, such models range from linear lumped models [23] to three-dimensional nonlinear simulations [24]. Once the model is calibrated, it provides a quantitative relation between the measured and unmeasured gas path parameters, and desired thermodynamic parameters from the model can be extracted as features $[25,26]$. The advantage of this approach is that the extracted feature can be defined with an insightful physical meaning in connection with GTE degradation.

\subsubsection{GTE Fault Detection and Diagnostics}

Degradation of the components manifest different symptoms in the GTE performance parameters. Once the useful features are extracted from the performance data, a decision making process can be set up to classify and quantify the invisible faults through their footprints in the features. Feature extraction is performed by the use of appropriate signal processing techniques, explained in section 1.2.2. The decision-making, referred to as diagnostics, is the procedure of mapping the extracted features from the feature space to the machine faults in the condition space.

The traditional approach of fault diagnostics is based on human expertise to observe the extracted features and conclude about the faults. This approach is usually time- 
consuming and unreliable, especially when multiple features are involved in diagnostics and the data are noisy [27]. A more advanced alternative is to use automatic diagnosis. Many automatic diagnostic techniques have been developed and utilized, which can be categorized into two main classes: model-based [28] and data-driven diagnostic methods [29]. Between the mentioned methods, there is a hybrid approach, which fuses both model-based mathematical models and data-driven models [30, 31]. Fusion methods aim to integrate the strengths of the model-based and the data-driven approaches at the same time, while alleviating their limitations.

Model-based methods typically involve building mathematical models to describe the state of the system and failure modes; they incorporate physical and behavioral understanding of the system into the estimation of system state [32]. Model-based approaches can be more effective than model-free approaches if an accurate model can be constructed [33]. However, explicit mathematical modeling may not be feasible for complex systems such as GTE, since it would be difficult to build an accurate mathematical model for such a system. Model-based approaches, also, may not be suitable for the industrial applications where the physical parameters and fault modes may vary under different operation conditions [34].

Data-driven methods include pattern recognition techniques and inference-based classifiers. The pattern recognition techniques include statistical classifiers, geometric methods, and polynomial classifiers [35], which have been implemented in various GTE diagnostic applications [36-39]. Many statistical classification approaches have been introduced that separate feature signals and group them into different fault categories or into healthy condition. A primary way for signal grouping is based on a defined distance measure or similarity measure between two signals. Such measures can be derived from a discriminant function in statistical pattern recognition [40]. Commonly used distance measures are Euclidean distance, Mahalanobis distance, Kullback-Leibler distance and Bayesian distance [41-44]. A powerful classification technique is called support vector machine (SVM), that constructs a decision hyperplane as the boundary between classes of given data by maximizing the distance of the closest point to the boundary [45]. SVM has found application in various diagnostic systems including GTE [46-52]. Hidden Markov model (HMM) can also be used for fault classification. HMM has been used to 
represent the evolution of the component's health condition by hidden states, using the features extracted from the measurement data [53].

Inference-based diagnostic classifiers employ artificial intelligent (AI) tools such as artificial neural networks (ANN) including multilayer, radial and dynamic ANN [54-57], fuzzy logic [58-60], and synergetic schemes [61, 62]. Classifiers using ANN have been broadly utilized for machine fault diagnosis. After being trained on available data, ANN can establish complex functional relations between inputs and outputs of a system, which are the features and the diagnosed faults in this case. However, their disadvantages are that their internal layers are obscure to the users, and there is no guarantee for their convergence during the training process [63]. Fuzzy logic classifiers mimic human reasoning to deal with the complexities of the system under study [64]. Fuzzy logic classifiers start with identification of structure of the problem and then utilize the expert knowledge formulated in a linguistic format. Diagnosis by fuzzy classifiers is conducted through sufficient number of fuzzy operations [65]. Fuzzy classifiers have found broad applications in machinery diagnostics including GTEs due to their effectiveness and concise form [60, 66-69]. The disadvantages with fuzzy systems are that they do not have effective learning capability and it is difficult to establish optimal inference rules when there are too many input variables [63]. The qualitative approach of the fuzzy models and the adaptive capability of ANN were combined into synergism schemes, known as neurofuzzy (NF) systems in early 1990s [70]. NF systems such as adaptive neuro-fuzzy inference system (ANFIS) benefit from an internal adaptation mechanism to adjust the fuzzy rule parameters through the training phase without a need to the expert knowledge required to design the conventional fuzzy systems [71]. Due to its advantages and effective performance, NF systems and ANFIS in particular have found many applications in GTE diagnostics [72-77].

Through the sequential fusion of the model-based and the data-driven methods, the hybrid method attempt to incorporate advantages of both, while alleviating their cons [78, 79]. In particular, physics-based models are usually constructed to connect the measurement parameters with the internal degradation state, while the state prediction model may be constructed on the physics of failure [80], or based on any mathematical model fitting [81-83]. The dynamic parameters of the system are then sequentially 
updated upon receiving new observations.

A number of model-data fusion frameworks were developed in various disciplines and some were applied to GTEs for degradation monitoring. For linear/Gaussian systems, Kalman filter (KF) has gained extreme publicity for its statistically optimal estimate in closed form [84]. More developments on KF have extended its application into nonlinear systems [85], and non-Gaussian noise distributions [86]. KF and its variants have been extensively used for performance monitoring and diagnostics [87, 88], however, their effectiveness is limited due to the need to linear/linearized state models and measurement models with known Gaussian structure for uncertainties and noise respectively. By emersion of powerful computational resources, particle filter (PF) was introduced for state estimation with no limitation for nonlinearity of the model or noise distributions. PF is a numerical method for sequential state estimation that applies on Monte Carlo sequential method to estimate the posterior distribution of the evolving state by a set of sample scenarios, which are represented by a set of particles and their corresponding assigned weights [89]. Some PF variants have been developed to improve the filtering performance [90-92], and this scheme is finding increasing number of applications for state estimation in nonlinear/non-Gaussian systems, including degradation monitoring and prognostics in GTEs [93-98].

Each of the aforementioned diagnostic techniques, however, has its own benefits and limitations and may outperform the others in particular applications [99]. When any diagnostic system is chosen for GTE health monitoring, the paramount issue is its reliability. Unreasonably missed positive (also called missed fault: i.e., the discriminator cannot detect an existing fault) and false positive (also called false alarm: i.e., the discriminator detects a non-existing fault because of the noise or other errors) can seriously mitigate the system's reliability [100].

\subsubsection{Degradation State Estimation and Prognostics}

GTE prognostics entails the use of current and previous machine conditions to predict the future health state of the system. There are two main prediction types aimed for prognostics. The first type, which is more widely used, is to predict how much time is left before a failure occurs, considering the current condition and past operation records. The 
time left before observing the next failure, i.e., the RUL is mathematically stochastic. Machine prognostics aims to figure out the distribution of RUL with this type of prediction [101, 102]. In some situations, especially when a failure is catastrophic, a second type of prediction is utilized, which is to predict the chance that a machine will operate without a failure until a given time in future. In this type of prediction, the target is to find joint distribution of the health state indices, given the current machine condition and past operation records [5, 103, 104].

From methodological point of view, prognostic techniques can be categorized in two main groups: physics-based methods and data-driven methods. Physics-based methods make use of physics laws to establish a time-based model to predict the growth of a fault [105]. The most commonly developed physics-based methods for prognostics are for crack propagation prediction [106, 107]. Another physics-based method studies the physics of failure using failure mechanism models to quantify the amount of damage incurred by the surrounding conditions on a component [80, 108]. Life consumption models employ underlying microstructural degradation models in GTE component level to predict RUL of the component and find consumed life of the system as a result [109, 110]. Although the failure mechanisms are known to be rooted in the degradation process of the component [111], it is usually impractical to directly measure such degradations. State estimation techniques are therefore required to estimate degradation of the parts by measuring their surrounding conditions, e.g., pressure, temperature, vibration and oil debris [112]. The measurement signals, which include the degradation symptoms will then be attributed to the root cause faults and the corresponding intensities [113]. In general, physics-based methods are limited to the cases where the degradation mechanism can be quantified at component level. The method cannot be easily used for complex systems where internal parameters are inaccessible for direct measurements by the available sensors.

Data-driven methods attempt to develop models based on the current and the past observed data from a system to predict future performance and RUL without relying on the physics laws describing failure mechanisms [114]. The observed data are deemed to contain valuable information about the unobservable degradation process with statistical correlations [78]. Directly observed data or indirectly inferred information can be used as 
features to quantify system degradation state as explained in section 1.2.2. The features may be a physical quantity such as the length of a crack in a blade [115], or only a calculated index from the measurements with no definite physical meaning, such as wavelet coefficients for time of arrival signal of the blades [116]. The future degradation state can then be predicted by identification of a degradation model. Data-dreiven methods can be categorized in two main groups: statistical methods, and artificial Intelligence (AI) tools [78]. The classical approaches for nonlinear time series predictions make use of statistical methods and stochastic process techniques such as the autoregressive (AR) model [117], the threshold AR model [118], the multivariate adaptive regression splines [119], the bilinear model [120], the Volterra series expansion [121], and the projection pursuit [122]. These approaches have found applications in machinery prognostics; however, it is usually difficult to develop an accurate statistical model for a complex mechanical system [123]. Recent interests in time series prediction have focused on the use of flexible AI tools, such as ANN [124-127] and fuzzy systems [128]. As stated in section 1.2.3, fuzzy systems lack a learning capability, and with increasing the number of input variables, it gets more difficult to establish the fuzzy forecasting inference rules. An effective solution to tackle this limitation is to use synergetic systems such as ANFIS where a forecasting operation is performed by fuzzy logic, and fuzzy system parameters are adjusted through training by ANN-based algorithms. Prediction schemes based on ANFIS were implemented for time series prediction and the simulation results demonstrated higher forecasting accuracy compared to those based on classical AR models, feed forward ANN and recurrent ANN [129-132].

Despite notable achievements in dynamic systems state prediction, the non-stationary operating condition of GTEs and diversity of the degradation mechanisms demand for more accurate holistic prognostic schemes.

\subsection{Thesis Objectives}

Structural complexity, non-stationary operating conditions and various recoverable and non-recoverable degradation mechanisms in the GTEs create an intricate prognostics problem, especially for the GTEs with no measurements available from their gas path parameters. To address the challenges, an advanced prognostic scheme, considering both 
the short-term and the long-term deterioration regimes is developed in this thesis with the following objectives:

1- To develop a novel physics-based modeling approach for GTE signal processing and feature extraction. The focus will be on performance deterioration monitoring in the GTEs with limited measurements available only from the operating systems with no additional instrumentation.

2- To develop time-variant state prediction models for both recoverable and nonrecoverable degradation under variable ambient conditions and operating profiles.

3- To develop system identification tools for nonlinear dynamical systems, e.g., GTEs, with stochastic inputs and outputs, in order to estimate unobservable degradation states.

\subsection{Experimental Data}

The experimental data used in this thesis have been collected from a $5 \mathrm{MW}$ single shaft GTE, which was in service for power generation in a local community for three years corresponding to the operating period between two major overhauls. Table 1.1 shows the basic technical specifications of the GTE in this study.

Table 1.1. Basic technical specifications of the GTE at the design point

\begin{tabular}{|l|c|}
\hline \multicolumn{1}{|c|}{ Parameter } & Value \\
\hline \hline Nominal power & $5(\mathrm{MW})$ \\
\hline Shaft speed & $16500(\mathrm{RPM})$ \\
\hline Thermal efficiency & $30.2(\%)$ \\
\hline Pressure ratio & 14.0 \\
\hline $\begin{array}{l}\text { Exhaust gas temperature } \\
(\boldsymbol{E G T})\end{array}$ & $785(\mathrm{~K})$ \\
\hline
\end{tabular}

Logging frequency from the GTE data was one reading every two hours during the entire working period. At each reading 44 parameters were logged from the GTE. The set of parameters comprises 3 control inputs, 22 measurements from the GTE accessory systems and 19 measurements from the gas path parameters. For the same period of time 
and the same location, the ambient condition data including air temperature, pressure and relative humidity have been received from Environment Canada, as shown in Table 1.2.

Table 1.2. Available ambient and operating parameters

\begin{tabular}{|l||c|c|c|c|c|c|c|}
\hline \multicolumn{1}{|l||}{ Source } & \multicolumn{3}{c|}{ GTE operating system } & \multicolumn{3}{c|}{ Environment Canada } \\
\hline Parameter & Power & $\begin{array}{c}\text { Shaft } \\
\text { speed }\end{array}$ & $\begin{array}{c}\text { Inlet } \\
\text { temperature }\end{array}$ & EGT & Temperature & Pressure & $\begin{array}{c}\text { Relative } \\
\text { humidity }\end{array}$ \\
\hline Count & 1 & 1 & 3 & 14 & 1 & 1 & 1 \\
\hline
\end{tabular}

\subsection{Thesis Outline}

A physics-based modeling approach for GTE is presented in Chapter 2 to process gas path measurement signals and to extract features, i.e., health indicators, with insightful physical meaning. The results clearly demonstrate the effectiveness of the developed approach, as well as the introduced indicators for GTE health monitoring.

Chapter 3 presents an enhancement in GTE physics-based model with taking into account the effects of air humidity. The results show improvements in the resulting indicator signal by reducing the noise and increasing the prediction accuracy.

In Chapter 4, a nonlinear/non-Gaussian data-model fusion framework is presented for long-term degradation state estimation of GTEs. The developed framework outperforms the existing regularized particle filter through simulation on a benchmark model, and by utilizing the real data from a GTE measurements.

An NF-based modeling approach is taken in Chapter 5 to construct a prediction model for short-term performance deterioration of the GTE due to compressor fouling. The results quantitatively describe the complex effects of humidity condensation rate on the GTE performance deterioration.

In Chapter 6, the nonlinear/non-Gaussian state estimation framework is extended to multivariate degradation state space, while the effects of stochastic inputs of the system are appropriately addressed in the mathematical model. The results demonstrated the effectiveness of the developed technique to extract two well-known non-recoverable degradation symptoms in the hot section of the GTE. 


\section{Chapter 2: A Physics-Based Modeling Approach for Performance Monitoring in Gas Turbine Engines ${ }^{1}$}

\subsection{Overview}

For a complex system like a GTE, the generic gas path analysis is an effective method for health monitoring and diagnostics using thermodynamic governing laws, however, most of the internal cycle parameters are hard to access and complex instrumentation is required for such measurements, which is not practically viable for typical operating ranges. It is desirable to develop diagnostic techniques, which take into account the effective parameters calculated from the measurement signals of the operating data for the GTE rather than the inaccessible internal parameters. For example, Sun et al. presented a weighted average indicator from sensor signals of the operating system to characterize the hidden health state of the system [105]. Despite many research works in this field, the effective methodologies are still needed to acquire reliable performance indicators from the signals available from GTE operating system. To develop such methods, it is important to consider that no single parameter can effectively indicate the degradation process of the entire system and many of the measured parameters convey no useful information about the degradation level. Through the use of physics-based modeling, this chapter aims to incorporate all the meaningful measured operating data from the GTE under typical service usage, while excluding the signals with no contribution. The objective of this chapter is to develop a physics-based performance deterioration monitoring approach that can clearly show the trends of short-term and long-term performance deterioration regardless of the operating conditions; the goal is to provide GTE operating industries with an effective tool to keep track of the degradation of their GTE for CBM without a need for additional hardware. The proposed technique is new in that it incorporates the following aspects: 1) the performance indicators introduced in the proposed approach are physically interpretable quantities that are

1: (C) 2014 IEEE. Reprinted, with permission, from [H. Hanachi, J. Liu, A. Banerjee, Y. Chen and A. Koul, "A Physics-based modeling approach for performance monitoring in gas turbine engine," IEEE Transactions on Reliability, November 2014] 
directly linked to the performance of the GTE as a system; 2) the indicators are applicable regardless of the ambient conditions and the operating profile of the GTE; 3) only the external parameters, which are readily available in every GTE operating system are utilized in the technique, and therefore no extra sensors are required; and 4) both recoverable and non-recoverable performance deteriorations can be characterized and addressed by the same indicators through appropriate trend analyses.

\subsection{Model Development}

The operating parameters measured from a GTE during service are interdependent in the way that variations in one parameter affect the others. GTE operating condition is a multivariate vector and the performance maps (if available) cannot easily cover all working scenarios to describe the relation between the operating parameters. A physicsbased heat balance model is therefore a useful tool to simulate all working conditions using the governing thermodynamic laws. Heat balance modeling is an effective approach at GTE system level and can be applied for GTEs with different layout designs. To construct and calibrate such a model, some design parameters of the GTE gas path and operating data of a freshly built GTE are required. This will enable the performance model to always simulate performance of a brand-new GTE and provide a baseline performance under varied operating scenarios. The deviation of the measured and predicted baseline performance can be further analyzed to monitor the performance deterioration with the indicators that provide physical insight into such changes. The more number of gas path parameters are available in the GTE measurements, the more number of indicators can be defined consequently for performance monitoring.

\subsubsection{The Thermodynamic Model for the Single Shaft GTE}

A single shaft GTE gas path comprises three main modules, the compressor, the combustion chamber(s) and the turbine. The basics of the gas path thermodynamic equations are available in the literature [133, 134]. In this part, the GTE gas path thermodynamic model is constructed by making a separate model for each module. The adjacent modules experience the same cycle parameters at their interface, e.g., the cycle 
parameters at the outlet of the compressor are equal to the inlet of the combustion chamber. The following steps show the procedure of the modeling.

The type and the design parameters of a compressor define its performance, i.e., the relation between corrected mass flow, corrected shaft speed, pressure ratio and isentropic efficiency, which are reflected in the compressor map. In order to facilitate the process of finding the map data, auxiliary coordinates, called beta lines are used. In the plane of the corrected mass flow and the pressure ratio, the beta lines are drawn between the choke line and the surge line with a proportional distance to each [9], as appear in Figure 2.1.

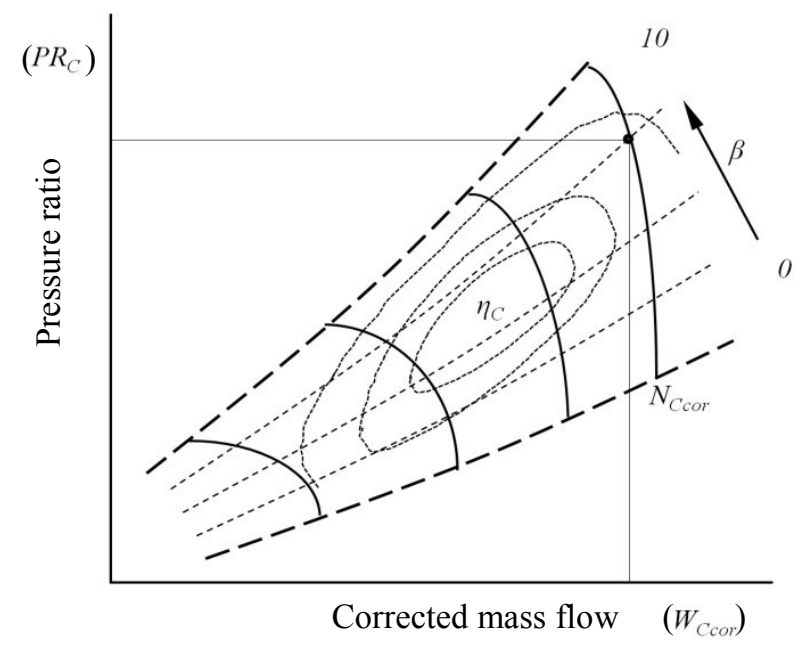

Figure 2.1. Schematic compressor map.

Given the intake air temperature and the shaft speed, and assuming an arbitrary beta line, the map returns the mass flow $W_{C}$, the pressure ratio $P R_{C}$ and the isentropic efficiency $\eta_{C}$ of the compressor, as pointed in Figure 2.1.

$$
\left[W_{C}, P R_{C}, \eta_{C}\right]=\operatorname{Map} p_{C}\left(N_{C_{c o r}}, \beta\right),
$$

where $N_{C_{c o r}}=N / \sqrt{T_{C i} / T_{s t d}}$ is the corrected shaft speed used in the map. Using the isentropic efficiency, the compressor outlet temperature is calculated from the inlet temperature and the compression ratio,

$$
T_{C o}=T_{C i}\left(\left(P R_{C}\left(\gamma_{A i r}-1\right) / \gamma_{A i r}-1\right) / \eta_{C}+1\right),
$$

and by using the mass flow, the required power of the compressor is given by, 


$$
P W_{C}=W_{C} c_{p_{A i r}}\left(T_{C o}-T_{C i}\right) .
$$

The specific heat capacity and heat capacity ratio are temperature dependent gas properties that are usually estimated by polynomials [135]. In a thermodynamic process, the average values of the mentioned parameters should be used in the respective equations.

In a GTE, the bleed flow $\left(W_{b}\right)$ is assumed to be a definite percentage of the compressor mass flow that bypasses the combustion chambers for cooling the nozzle and the blades of the turbine. The rest of the compressor mass flow enters the combustion chambers and mixes with the fuel with the ratio of $f=W_{F} / W_{C h}$, where $W_{C h}=W_{C}-W_{b}$ and $W_{F}$ is the fuel mass flow. The temperature of the combustion product from the first law of thermodynamics is

$$
T_{C h o}=f\left(c_{p_{F}}\left(T_{F}-T_{C o}\right)+L C V\right) /\left((1+f) c_{p_{P}}\right)+T_{C o},
$$

where $L C V$ is the lower calorific value of the fuel and $c_{p_{P}}=c_{p_{A i r}}+f /(1+f) \theta_{c_{p}}$ is the specific heat capacity of the combustion product $[136,137]$.

At the nozzle and the turbine blades, the cooling air dilutes the combustion product and lowers the temperature to

$$
T_{T i}=\left(W_{C h} c_{p_{P}} T_{C h o}+W_{b} c_{p_{A i r}} T_{C o}\right) /\left(W_{C h} c_{p_{P}}+W_{b} c_{p_{A i r}}\right) .
$$

Equation (2.5) is valid when the turbine has more than one stage, and the energy of the cooling air of the first stage can be extracted in the next stages. This applies for the subject turbine in this research. The turbine pressure ratio is calculated from the compressor pressure ratio and the pressure drop ratios in the intake filter, the combustion chamber and the exhaust duct:

$$
P R_{T}=P R_{C} P R_{F i l} P R_{C h} P R_{E x h} .
$$

The turbine map defines the relations between corrected mass flow, corrected shaft speed, pressure ratio and isentropic efficiency. Taking the same shaft speed of the compressor, the turbine map returns the mass flow and the isentropic efficiency:

$$
\left[W_{T}, \eta_{T}\right]=\operatorname{Map}_{T}\left(T_{T i}, P_{T i}, N, P R_{T}\right),
$$

where, 


$$
P_{T i}=P_{C i} P R_{C} P R_{C h}
$$

is the pressure at the turbine inlet.

The system of equations from (2.1) to (2.8) should be solved through iterations by revising $\beta$ in (2.1), until the mass conservation equation holds, i.e., $W_{T}=W_{C}+W_{F}$. With the acquired mass flow and isentropic efficiency of the turbine, the outlet temperature and the power of the turbine are calculated:

$$
\begin{aligned}
& E G T=T_{T i}\left(\eta_{T}\left(P R_{T}^{\left(1-\gamma_{M i x}\right) / \gamma_{M i x}}-1\right)+1\right), \\
& P W_{T}=W_{T} c_{p_{\text {Mix }}}\left(T_{T i}-E G T\right),
\end{aligned}
$$

where $\gamma_{\text {Mix }}$ is the heat capacity ratio and $c_{p_{\text {Mix }}}$ is specific heat capacity of the mixture of the combustion product and the cooling air.

A portion of the turbine power runs the compressor. The net output power of the GTE is therefore:

$$
P W_{G T E}=P W_{T}-P W_{C}-P W_{L},
$$

where $P W_{L}$ is the sum of the internal losses that is estimated about one percent of the compressor power [133].

Equations (2.1) to (2.11) constitute the thermodynamic model of the single shaft GTE, which can be expressed in the following compact form using the external parameters of the GTE:

$$
\operatorname{Map}_{G T E}\left(T_{C i}, P_{C i}, N, P W_{G T E}, E G T\right)=0 .
$$

Mathematically, the system of equations is determined when as many as four independent cycle parameters are known. In other words, in a given ambient temperature and pressure, the developed thermodynamic model can determine all the unknown GTE cycle parameters using only two known cycle parameters in (2.12).

\subsubsection{Performance Indicators from the Over-Determined Model}

The operating data measured in an industrial GTE often include the shaft speed, the power and EGT. As explained in 2.2.1, two of the measured parameters are sufficient to determine the output of the proposed model using (2.12), and so the additional 
parameters over-determine the model. In a GTE with normal performance, the expected $E G T\left(E G T_{E x p}\right)$ can be calculated from (2.12) when the shaft speed and the output power are available:

$$
\begin{aligned}
E G T_{E x p} & =E G T_{E x p}\left(T_{C i}, P_{C i}, N, P W_{G T E}\right) \\
& =E\left[E G T \mid F_{G T E}=0\right] .
\end{aligned}
$$

There are different degradation mechanisms such as changes in blade surface due to erosion or fouling and changes in clearances due to abrasion that cause performance deterioration in the GTE [138]. In such conditions, the actual gas path geometry deviates from the ideal design condition and (2.14), which is based on brand-new design parameters becomes less accurate. In fact, when the performance of the GTE deteriorates, it becomes unable to extract the heat energy as much as in the healthy condition, and the EGT rises as the result. The magnitude of the EGT rise depends primarily on the GTE degradation level, because the operating condition is accounted in the thermodynamic model. For the performance monitoring, we define the heat loss index $\left(I_{H L}\right)$ as the ratio of the EGT rise with respect to the design point $E G T$ :

$$
I_{H L}=\left(E G T-E G T_{E x p}\right) / E G T_{D} .
$$

To acquire another performance indicator, one can solve (2.12) for the GTE expected power, using the shaft speed and the EGT from the operating system:

$$
\begin{aligned}
P W_{E x p} & =P W_{E x p}\left(T_{C i}, P_{C i}, N, E G T\right) \\
& =E\left[P W_{G T E} \mid F_{G T E}=0\right] .
\end{aligned}
$$

In a real working condition, the power gradually declines from the expected power of a healthy GTE due to the performance deterioration. The magnitude of the loss of power is primarily depended on the degradation level, because the thermodynamic model considers the operating condition. For the performance monitoring, the power deficit index $\left(I_{P D}\right)$ is defined as the ratio of the power deficiency to the design point power:

$$
I_{P D}=\left(P W_{E x p}-P W_{G T E}\right) / P W_{D} .
$$

As explained earlier, the EGT exceeds its expected value with the GTE performance deterioration, which leads to an increase in $I_{H L}$. Similarly, the performance deterioration 
causes a deficiency in the GTE power, compared with the ideal condition. Consequently, $I_{P D}$ will rise as the result of the performance deterioration. In this regard, both indices have a positive correlation with the degradation process of the GTE.

\subsection{Model Implementation}

For complex engineering systems such as GTEs, the performance deterioration is a major concern that gets in the way of reliable and economical operation of the system. In order to ensure reliable and cost effective operation of a GTE, it is critical to identify the present and the future health state of the GTE by monitoring the performance deterioration and the underlying structural degradation. In the proposed physics-based performance monitoring approach, the performance indicators provide quantitative measures for the degradation process. To assess the effectiveness of the developed performance indicators for deterioration monitoring, a set of 3-year operating data with 12 readings per day from a $5 \mathrm{MW}$ power generation GTE is used. At each reading 44 parameters were logged from the GTE including 19 measurements from GTE gas path parameters. There are a number of redundancies in the temperature measurement for the inlet air and the outlet exhaust gas. Eliminating the redundancies and using respective average values, the parameters $T_{C i}, P_{C i}, N, P W_{G T E}$ and the EGT are the usable cycle parameters to find the thermodynamic based performance of the GTE using the developed performance indicators in 2.2.2.

In many occasions during the time between overhauls (TBO), the cycle parameters were not logged due to the GTE shut down for maintenance and service. Such time steps were left as blanks in the digitized data file. To eliminate the wrong entries in the data logging and the digitization, the graphical tool was employed and the outliers, which were physically unreasonable, were removed from the data.

To establish the physics-based relation between the cycle parameters, a computer code was written in MATLAB environment for the developed model discussed in section 2.2. The code is structured to employ the performance maps of the compressor and the turbine in tabular format as in the following.

- The performance map of the compressor is available in tabular format for the operating speed range and $\beta$ values. Table 2.1 shows some sample data from the GTE 
compressor in this study.

Given the inlet air temperature and the shaft speed, the corrected speed ratio of the compressor is,

$$
N_{C_{c o r}} / N_{D}=N /\left(N_{D} \sqrt{T_{C i} / T_{s t d}}\right),
$$

where $T_{s t d}=288.15 \mathrm{~K}$ is the standard atmosphere temperature and $N_{D}=16500 \mathrm{RPM}$ is the shaft speed at the design point for the GTE in this study. Using a bilinear interpolation on the corrected speed ratio and an arbitrary $\beta$, the table returns the corrected mass flow, the pressure ratio and the isentropic efficiency of the compressor.

With a known inlet pressure, the mass flow of the compressor is found by,

$$
W_{C}=W_{C_{c o r}}\left(P_{C i} / P_{s t d}\right) / \sqrt{T_{C i} / T_{s t d}},
$$

where $P_{\text {std }}=101.325 \mathrm{kPa}$ is the standard atmosphere pressure. In this way, the compressor map provides its outputs as parameterized in (2.1).

Table 2.1. Sample data from the compressor map

\begin{tabular}{ccccc}
\hline \hline & $N_{C_{c o r}} / N_{D}$ & $W_{C_{c o r}}(\mathrm{~kg} / \mathrm{s})$ & $P R_{C}$ & $\eta_{C}$ \\
\hline 0 & 0.85 & 13.689 & 6.191 & 0.8149 \\
1 & 0.85 & 13.645 & 6.634 & 0.8208 \\
2 & 0.85 & 13.588 & 7.168 & 0.8311 \\
3 & 0.85 & 13.486 & 7.653 & 0.8386 \\
4 & 0.85 & 13.428 & 8.074 & 0.8472 \\
\hline
\end{tabular}

- The performance map of the turbine is extracted from the performance table in a similar approach. Some sample performance data from the GTE turbine are shown in Table 2.2.

If the turbine inlet temperature and pressure, the shaft speed and the turbine pressure ratio are all given, the corrected speed ratio of the turbine is

$$
N_{T_{c o r}} / N_{D}=N /\left(N_{D} \sqrt{T_{T i} / T_{T i_{D}}}\right),
$$

where $T_{T i_{D}}=1323 \mathrm{~K}$ is the turbine inlet temperature at the design point. In a known pressure ratio, a bilinear interpolation on the corrected speed ratio and the pressure ratio provides the corrected mass flow and the isentropic efficiency of the turbine. The mass 
flow is then calculated by

$$
W_{T}=W_{T_{c o r}} N_{D}\left(P_{T i} / P_{s t d}\right) /\left(N_{T_{c o r}} \sqrt{T_{T i} / T_{s t d}}\right),
$$

Hence, the performance map data are extracted from the performance table as described by (2.7).

Once the performance maps are implemented into the code, the model equations can be solved in either forms of (2.13) or (2.16) depending on whether we are looking for the heat loss index or the power deficit index, respectively. Figure 2.2 depicts the flowchart of the modeling process, in which the path numbered 1 is taken to calculate $E G T_{E x p}$, when the heat loss index is required, and the path numbered 2 is the way that the code calculates $P W_{E x p}$, in order to find the power deficit index.

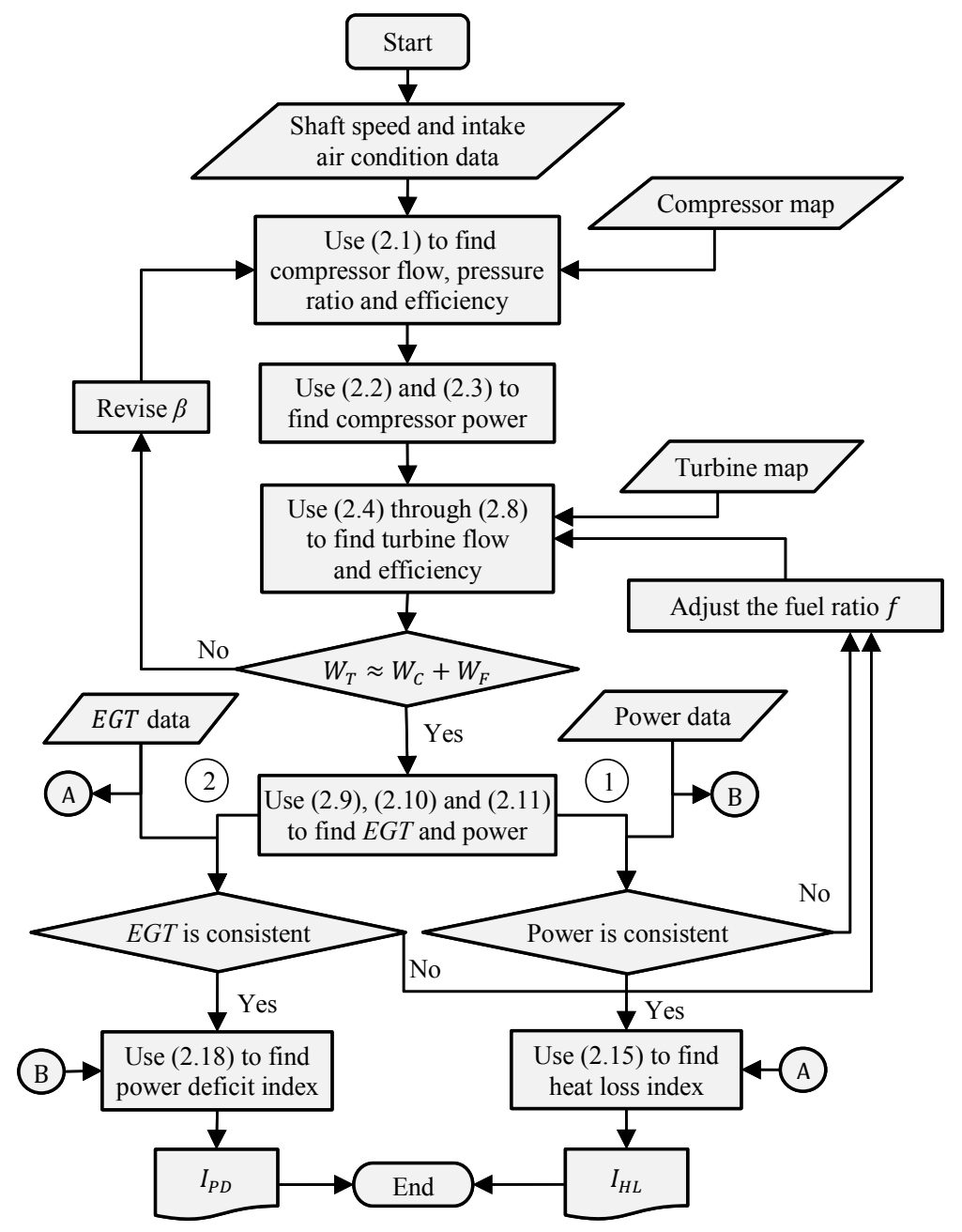

Figure 2.2. The model calculates both the heat loss index and the power deficit index. 
Table 2.2. Sample data from the turbine map

\begin{tabular}{ccccc}
\hline \hline$\beta$ & $N_{T_{\text {cor }}} / N_{D}$ & $W_{T_{\text {cor }}}(\mathrm{kg} / \mathrm{s})$ & $P R_{T}$ & $\eta_{T}$ \\
\hline 6 & 0.95 & 2.6518 & 11.401 & 0.8773 \\
7 & 0.95 & 2.6516 & 12.552 & 0.8711 \\
8 & 0.95 & 2.6534 & 13.636 & 0.8634 \\
9 & 0.95 & 2.6541 & 14.787 & 0.8503 \\
10 & 0.95 & 2.6549 & 15.916 & 0.8354 \\
\hline \hline
\end{tabular}

The developed model has been verified using the set of data from the GTE operating system. It especially shows much reliable performance to predict the trend of variation of the operating parameters. This will be further discussed in the following section.

\subsection{Analysis}

\subsubsection{The Heat Loss Index Trend Analysis}

The average EGT was acquired from the temperature sensors at the GTE exhaust. As seen in Figure 2.3(a), EGT has been changing during the operating period due to the variation of the working condition, e.g., power demand and weather temperature. The values of $T_{C i}, P_{C i}, N$ and $P W_{G T E}$ were also logged for each time step. These parameters were used in the GTE model to calculate the expected EGT each time step as plotted in Figure 2.3(b). The discontinuities in the plots correspond to the periods when the GTE was shut down for maintenance.

An overall comparison between the plots depicts similar patterns for the whole operating time. The similarity is more observable in the trend of the plots rather than the absolute values, e.g., from day 500 to 600 and day 850 till 950, the expected EGT follows similar slump patterns as observed in the measured one. The slumps correspond to the summer times when the power demand from the GTE was lower. Nevertheless, a slight offset between the measured and the expected EGT is observable. That is because the generalized maps are used for the compressor and the turbine in the modeling. The maps are calibrated with the published cataloged design parameters of the GTE to reproduce the same behavior of the original compressor and turbine, however small mismatches in 
the maps can cause an offset in the GTE model behavior. Some experiments with different calibration parameters were conducted, which showed that in all cases the offset between the calculated and the measured parameters vary, while the self-relative ratios of $E G T_{E x p}$ remain acceptably unchanged, which is promising for trend analysis.

(a)

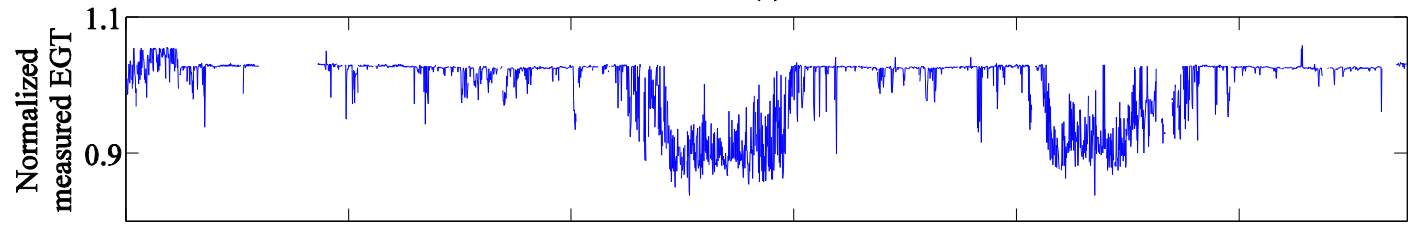

(b)

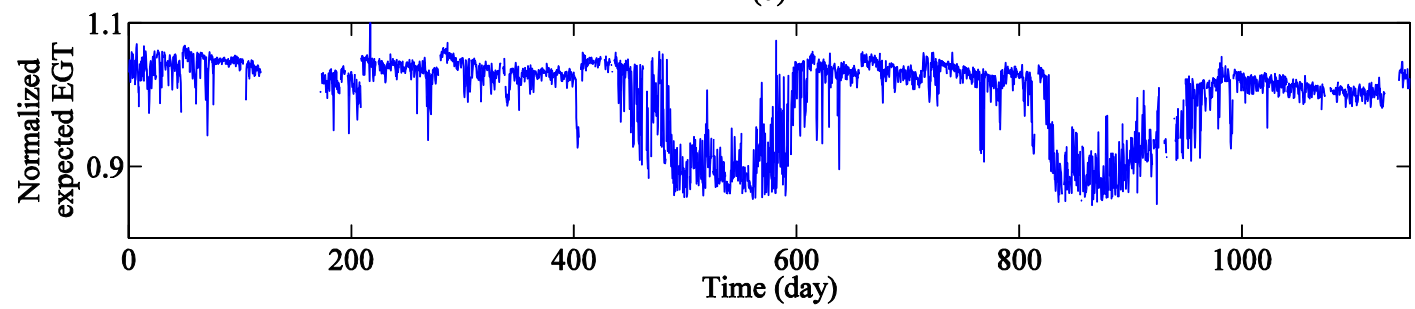

Figure 2.3. Variation of $E G T$ throughout the operating time: (a) the normalized measured $E G T$, and (b) the normalized expected $E G T$.

From the measured $E G T$ and the expected $E G T$, the heat loss index was calculated for each time step. Figure 2.4(a) illustrates the variations of $I_{H L}$ during the operating time of the GTE. It shows that $I_{H L}$ is noise contaminated, though the trend of changes is clearly visible. This noise is attributed to the measurement noise with the sensors of the operating system and inevitable uncertainties in modeling the GTE. To eliminate the noise from the signal, a moving average on a window of 15 time steps is implemented. This window size is large enough to remove the spikes, yet small enough to show local variations. The filtered signal can better show the trend of changes as appears in Figure 2.4(b). Two distinct patterns are observable in the plot as explained in the following.

In the short-term, $I_{H L}$ ascends with a steep trend and then falls to almost its initial level. The saw-tooth behavior repeats in the intervals varying between 50 to 120 days. It implies that the GTE performance deteriorates in the short intervals, and then suddenly recovers at the end of each interval. The observation complies with the knowledge about performance variation due to compressor fouling and compressor wash [139]. By 
investigating the service records of the GTE, it was found that the GTE compressor was washed at the same dates of the performance sudden changes. The extent of performance retrieval depends on two major factors. The longer the time between compressor washes, the more fouling material congests on the compressor blades. As a result, the performance retrieval depends on how long the compressor has worked since the last wash. In addition, the washing quality is the other effective factor in performance retrieval [140].

(a)

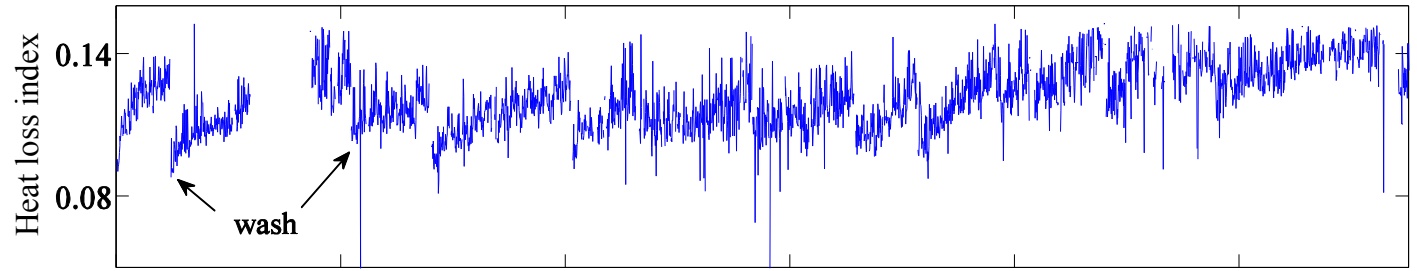

(b)

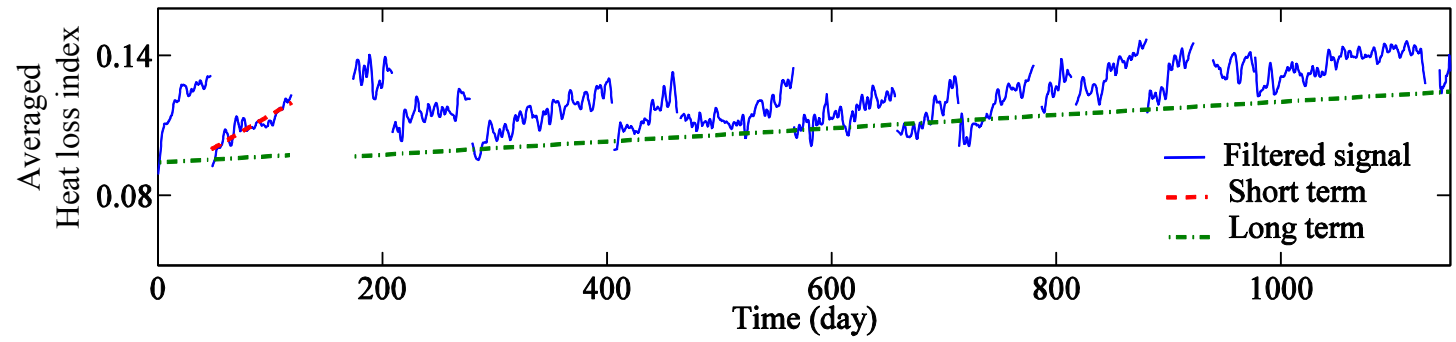

Figure 2.4. The heat loss index shows distinct short-term and long-term variation trends: (a) the original signal, and (b) the filtered signal.

For a GTE with selected air filter type, the rate of compressor fouling depends on the pollution intensity of the intake air and the mass flow rate [141]; therefore, the rate of the short-term variations in the heat loss index is not constant. To find the overall variation rate of $I_{H L}$, a linearized model is employed, which can be acquired by linear regression on the index values between two consecutive compressor washes:

$$
\widehat{I}_{H L}=\alpha_{0}+\alpha_{1} t_{k} \quad k=m, \ldots, n,
$$

where,

$$
\begin{aligned}
& \alpha_{1}=\sum_{m}^{n}\left(t_{k}-\bar{t}\right)\left(I_{H L_{k}}-\overline{I_{H L}}\right) / \sum_{m}^{n}\left(t_{k}-\bar{t}\right)^{2}, \\
& \alpha_{0}=\overline{I_{H L}}-\alpha_{1} \bar{t} .
\end{aligned}
$$


Equation (2.23) estimates $I_{H L}$ by a linear model, where $\alpha_{1}$ is the rate of variation between time steps $\mathrm{m}$ and $n$. Table 2.3 gives the calculated variation rates of $I_{H L}$ in selected washing periods. The average short-term variation rate of the heat loss index equals $245 \times 10^{-6}$ units/day for the whole operating time. This rate can be interpreted as the GTE performance has been deteriorating about $1 \%$ per 40 days.

In the longer term, the average of $I_{H L}$ increases slowly throughout the operating time. It reflects the GTE performance deterioration because of different failure mechanisms in the GTE parts and sub-systems [142] related to irreversible structural degradation. To track the long-term changes of the heat loss index and eliminate the effects of compressor fouling, the data was sampled from the first three days after each compressor wash, when the compressor is presumably clean enough to not affect the performance results. Imperfect wash of the compressor does not fully remove the fouling, nor its effects on the performance. Such effects may appear as noise in the trend of the long-term performance variations. Similar to the short-term variations, to find the overall trend, a linear model for the long-term changes of $I_{H L}$ is assumed. It is known that the GTE was shut down between days 119 and 172, during which it has not been degrading. In this regard, only the working times of the GTE have been considered to find the regression model for the long-term trend. The result shows a growth rate of $29.2 \times 10^{-6}$ units/day for $I_{H L}$. This result means that the long-term increase of the heat loss index is about 8.4 times slower than the short-term one. The long-term changes of $I_{H L}$ correspond to non-recoverable structural degradations of the GTE components; and to retrieve the normal performance,

the degraded parts should be repaired or replaced. That was the reason to replace the GTEs in each three-year period, and overhaul them in the supplier's plant.

\subsubsection{The Power Deficit Index Trend Analysis}

Among the measured parameters by the GTE operating system, the values of $T_{C i}, P_{C i}$, $N$, and EGT can be used in the model to find the expected GTE power using (2.16). The model predicts the value of the power output given the ideal performance condition of the GTE. At the same time steps, the measured value of the GTE power is included in the logs. Figure 2.5 depicts the measured power, and the expected power of the GTE, normalized with respect to their average values. The plots show an overall similarity in 
the trends; however, a minor offset between the two plots is observed. Similar to the case of the EGT, the offset is caused by using the generalized maps for the compressor and the turbine.

(a)

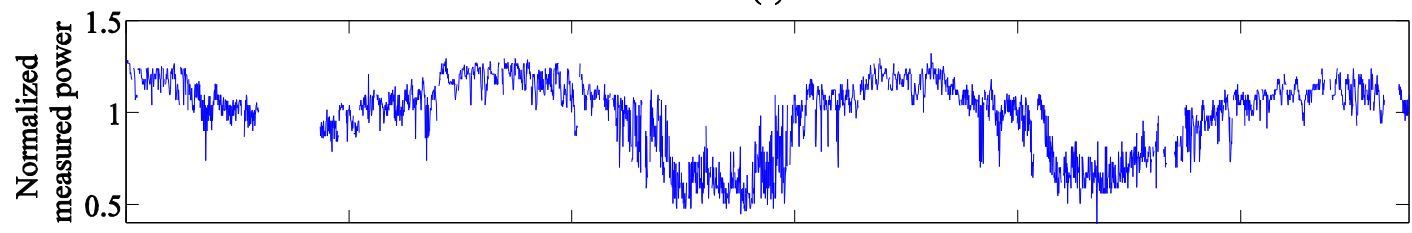

(b)

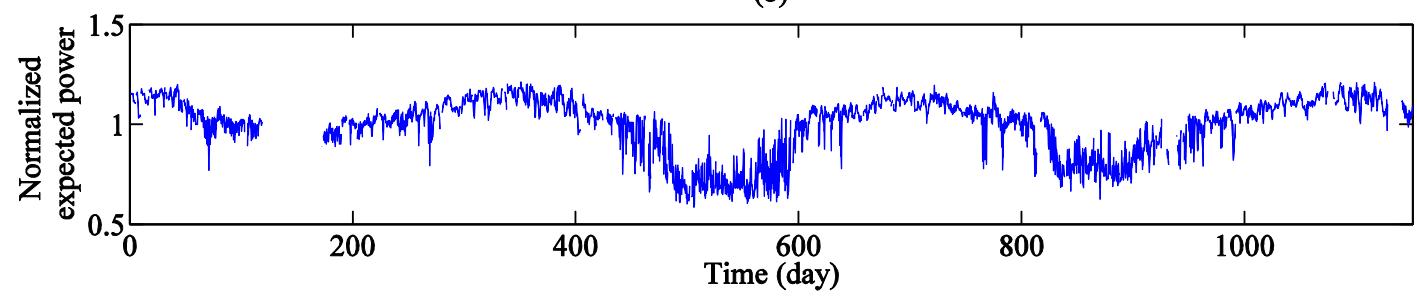

Figure 2.5. Variation of the GTE output power: (a) the normalized measured power, and (b) the normalized expected power.

In the course of the GTE operation, we expect to see performance deterioration compared to the baseline condition. In other words, the GTE is supposed to generate less power as it degrades further. Equation (2.18) quantifies the level of degradation using the power deficit index, which is the normalized difference between the actual and the expected power. The normalization is done with respect to the GTE rated power. Figure 2.6 depicts the variation of $I_{P D}$ during the operating time of the GTE. Similar to the heat loss index, the results clearly display a saw-tooth pattern for $I_{P D}$ variations.

The short-term variations of $I_{P D}$ happen in exactly the same time intervals as that of $I_{H L}$, i.e., the points of the sudden falls are the dates of compressor washes. Similar deficiencies in GTE power due to compressor fouling has been reported in the literature [143]. Assuming a linear model for the overall effect of the compressor fouling on $I_{P D}$ in short intervals, the trend of the fouling is quantified by linear regression on the index values using (2.23) for $I_{P D}$. As stated in Section 2.4.1, it is known that the fouling rate may vary depending on intake air pollution intensity and the mass flow rate. In Table 2.3, the variation rates of $I_{P D}$ in selected short-term periods are presented. On average, the 
short-term variation rate of the index is $495 \times 10^{-6}$ units/day.

(a)

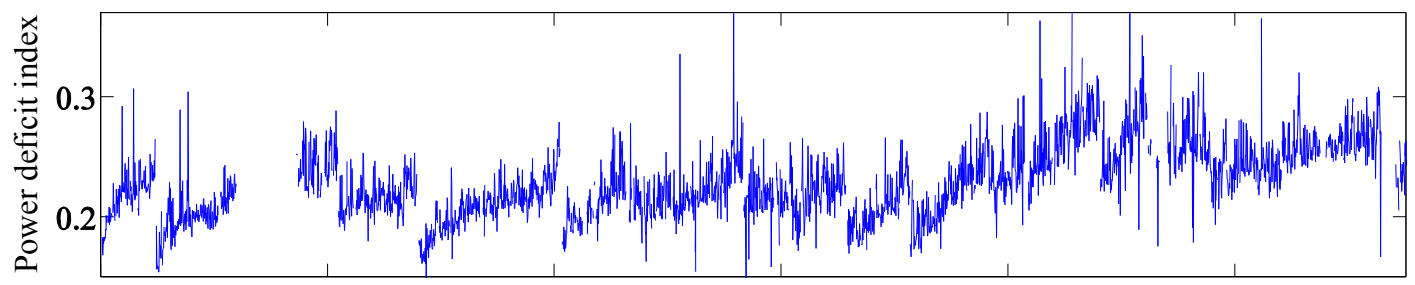

(b)

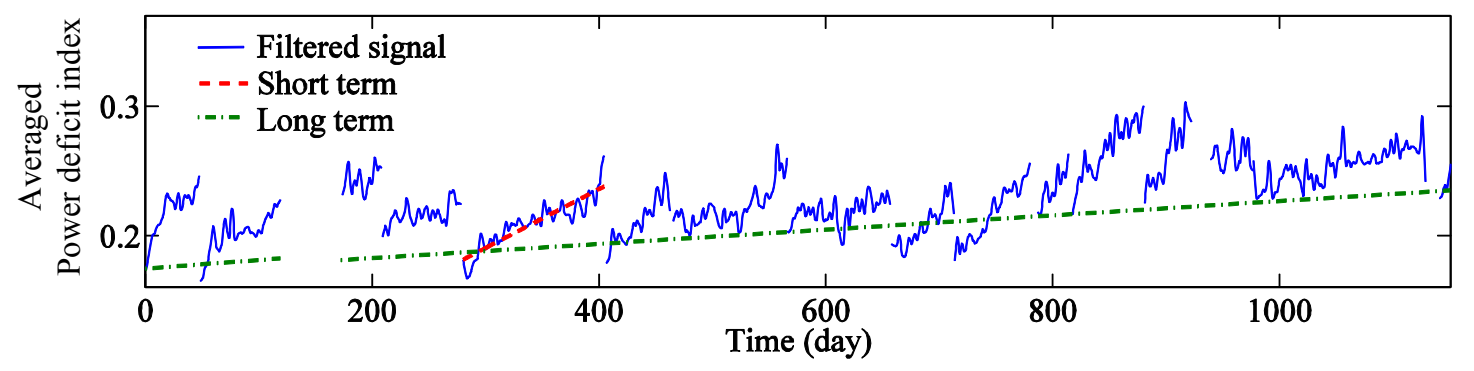

Figure 2.6. The short-term, and the long-term variation trends are observable in the power deficit index: (a) the original signal, and (b) the filtered signal.

Table 2.3. Variation rate of the performance indicators in selected periods

\begin{tabular}{ccc}
\hline \hline $\begin{array}{c}\text { Time interval } \\
(\text { days })\end{array}$ & $\begin{array}{c}I_{H L} \\
\left(10^{-6} \text { unit/day }\right)\end{array}$ & $\begin{array}{c}I_{P D} \\
\left(10^{-6} \text { unit/day }\right)\end{array}$ \\
\hline Short-term & & \\
$465-565$ & 112 & 296 \\
$658-712$ & 404 & 811 \\
$881-932$ & 358 & 739 \\
$939-976$ & 126 & 36 \\
average & 245 & 495 \\
Long-term & & \\
$1-1144$ & 29.2 & 62.2 \\
\hline \hline
\end{tabular}

To investigate the long-term performance deterioration of the GTE, the data were sampled from the first three working days after each compressor wash, and only the working days of the GTE were considered for trend modeling. The variation rate of $I_{P D}$ was calculated through linear regression. The result shows a long-term growth rate of 
$62.2 \times 10^{-6}$ units/day for the index. It shows that the long-term performance deterioration is about 8 times slower than the short-term one in the scale of $I_{P D}$. The results satisfactorily comply with the results of the $I_{H L}$ trend analysis.

\subsubsection{A comparative Study on the Performance Indicators}

Both the developed performance indicators demonstrate their effectiveness to display the track of the deterioration. Figure 2.7 compares the short-term average variation rates of the indices between two consecutive compressor washes by linear regression. The short-term variation rates of both indices periodically fluctuate throughout time, which implies that the fouling rate of the compressor speeds up and slows down sequentially. Noticing the GTE power variations in Figure 2.5(a), we observe that the compressor fouling rate follows a pattern similar to that of the power, i.e., the fouling happens faster when the GTE is generating higher power. This effect can be explained by the fact that more power generation by a GTE is associated with more air mass flow thorough the compressor, which results in a faster fouling of particles on the blades. There are two occasions where the variation rates of the indices do not agree: the variation rate of $I_{H L}$ implies that the compressor fouling becomes faster after the sixth wash (point i), and a bit slower after the 13th wash (point ii). However, the variation rate of $I_{P D}$ tells an opposite story for the same cases. Therefore, we should investigate which of the performance indicators is more reliable at each point.

The piecewise linear regressions of the indices provide time dependent models to estimate the performance deterioration. As appears in Figures 2.4 and 2.6, the data points are scattered around the regression lines. The error of the regression model for $I_{H L}$ at time step $k$ is

$$
\varepsilon_{k}=I_{H L_{k}}-\widehat{I}_{H L_{k}}, \quad k=m, \ldots, n .
$$

The standard deviation of the errors provides a measure for overall modeling certainty [144]. When comparing the two different models, the normalized errors should be used to eliminate the scale of the measuring units:

$$
\text { Modeling } \text { error }_{H L}=\sqrt{\sum_{m}^{n} \varepsilon_{k}^{2} /(n-m+1)} / \overline{I_{H L}} \text {. }
$$


The modeling errors calculated for the time segments between the consecutive compressor washes are plotted in Figure 2.7 for both indices. The normalization is done with respect to the average values of the respective indices in the same time intervals. For the two occasions (points $\mathrm{i}$ and ii), the modeling errors are slightly less with $I_{H L}$; therefore, when the linear model is adopted, the performance deterioration is more likely to follow the pattern described by this indicator.

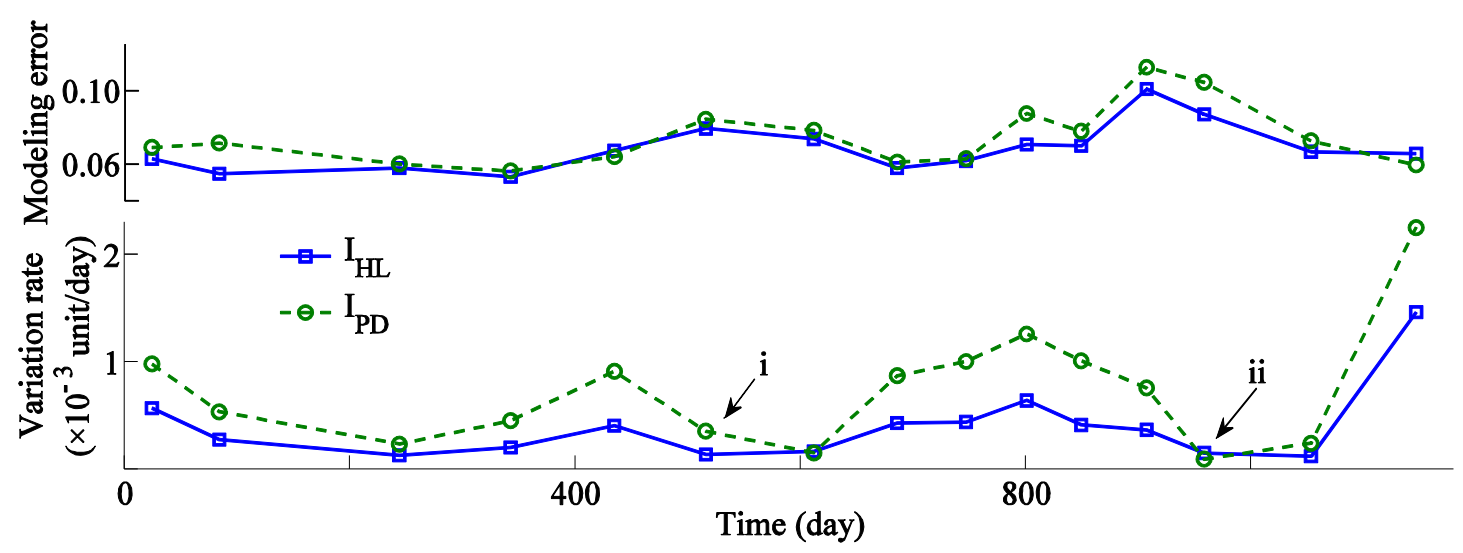

Figure 2.7. The short-term variation rate of the performance indicators fluctuate throughout the operating time.

Table 2.4 gives the average modeling errors for the short-term, and the long-term variation of the indices. The results suggest that both $I_{H L}$ and $I_{P D}$ can acceptably formulate the pace of performance deterioration; however, $I_{H L}$ shows slightly better accuracy in both cases. In addition, it shows that the linear regression on the indices can provide more accurate models for the short-term deterioration than the long-term. This difference can be caused by the fact that the quality of the compressor wash is dependent on the skill of the operators and their available time. If the compressor is not washed perfectly, the performance of the GTE will still be affected by the compressor fouling. This effect will appear as noise in the long-term deterioration trend, and reduce the modeling accuracy. 
Table 2.4. Modeling error

\begin{tabular}{ccc}
\hline \hline & $I_{H L}$ & $I_{P D}$ \\
\hline Average short-term & 0.0637 & 0.0698 \\
Long-term & 0.0913 & 0.1049 \\
\hline \hline
\end{tabular}

\subsection{Summary}

A physics-based model for single shaft GTE was developed in this chapter to calculate the performance parameters using typical measurements from the operating system. The model was calibrated with the performance of a freshly built GTE, and then utilized to calculate the baseline values of the exhaust gas temperature and the power of the GTE. Two performance indicators were proposed for GTE degradation monitoring, based on the deviation of the measured and the calculated performance parameters. The indicators have explicit physical interpretations, i.e., the heat loss index gives a measure of the excessive exhaust gas temperature, and the power deficit index gives the proportion of power deficiency caused by the GTE performance deterioration. The strength of the indicators is in that they are not functionally dependent on the operating points and the ambient conditions. The technique is especially advantageous in that it uses only the measurements from the GTE operating system without a need to additional sensors.

The developed method was verified using data from an industrial GTE. The results showed that both indicators could clearly reveal the short-term variation of the performance due to compressor fouling and consequent wash. They could also display the gradual degradation of the GTE during the operating time. The variation trends of the indicators and their occasional sudden changes were thoroughly in agreement with the background knowledge about the performance of GTEs and the information from the service logs of the machine in the study. The observations clearly show the effectiveness of the physics-based approach for performance monitoring using the limited measurements from the operating system. 


\section{Chapter 3: Effects of Intake Air Humidity on the Gas Turbine Performance Monitoring}

\subsection{Overview}

In Chapter 2, a physics-based modeling approach was introduced to develop unified performance indicators, using the basic measurements from the GTE operating systems. The goal was to reflect the overall health level of a GTE with a single index, independent from its operating conditions. One factor in the ambient condition is the humidity of air. Although the mass ratio of the moisture to air is often less than $2 \%$, due to the large difference between the thermodynamic properties of water vapor and air, the corresponding effects should be considered in the performance of the GTE [145]. To take into account the effects of the humidity, different methods such as dimensionless parameter groups for overall engine performance have been utilized by some researchers [9]. Our investigation showed that the mentioned simplified approach was not satisfactorily accurate to be used for the performance indicators. Some studies have taken into account the humidity effects in the component level, and found the percentage of deviations for selected performance parameters [146]. To improve performance monitoring accuracy with the performance indicator developed in Chapter 2, in this chapter a detailed thermodynamic model is constructed, which takes into account the effects of the humidity in each individual sub-assembly of the GTE. The objective is to provide GTE performance indicators, which have the highest achievable accuracy with regard to the available measurements from the operating system.

\subsection{Gas Turbine Model with Humid Air}

To study the performance of a GTE, in the previous chapter, a detailed thermodynamic model for the single shaft GTEs was developed and presented. In the developed primary model, the properties of dry air were utilized. The closed form of the developed model to predict the power $(P W)$ and the $E G T$ were presented in the following forms: 


$$
\begin{aligned}
& P W_{G T E_{M}}=P W_{G T E_{M}}\left(T_{C i}, P_{C i}, N, f\right), \\
& E G T_{M}=E G T_{M}\left(T_{C i}, P_{C i}, N, f\right),
\end{aligned}
$$

in which $T_{C i}$ and $P_{C i}$ are the temperature and the pressure of the intake air, $N$ is the shaft speed and $f$ is the fuel to air mass ratio. For a more accurate model however, the thermodynamic effects of the humidity of the intake air should be taken into account in the GTE model. A simplistic approach is to modify the overall engine model, using dimensionless parameter groups [9], where the dimensionless group for the power of a GTE, using a working fluid with the constant $R$ and the specific ratio $\gamma$ is: $P W /$ $\left(\gamma P_{C i} \sqrt{\gamma R T_{C i}}\right)$. In practice, both the mass flow and the properties of the working fluid will change due to bypassing of the bleed air, addition of the fuel, and creation of the combustion products. Therefore, a detailed model is needed to take into account such variations in each part of the GTE.

Compressor: For the compressor, the map provides the corrected mass flow, the pressure ratio and the isentropic efficiency, given the corrected shaft speed and the auxiliary $\beta$ line $[25,26]$ :

$$
\left[W_{C_{c o r}}, P R_{C}, \eta_{C}\right]=\operatorname{Map}_{C}\left(N_{C_{c o r}}, \beta\right)
$$

The corrected shaft speed, when the intake air is humid with $T_{C i}$ temperature is [9]:

$$
N_{C_{c o r}}=N \sqrt{\gamma_{a} R_{a} T_{s t d}} / \sqrt{\gamma_{h} R_{h} T_{C i}}
$$

where $T_{s t d}=288.15 \mathrm{~K}$ is the standard atmosphere temperature. $R_{h}$ and $\gamma_{h}$ are the gas constant and the specific heat ratio for the humid air, and they can be found by:

$$
\begin{aligned}
& R_{h}=\left(R_{a}+R_{w} S H_{C i}\right) /\left(1+S H_{C i}\right), \\
& \gamma_{h}=c_{p_{h}} /\left(c_{p_{h}}-R_{h}\right),
\end{aligned}
$$

where $S H_{C i}$ is the specific humidity of the intake air, and $c_{p_{h}}$ is the specific heat capacity of the humid air, found from the following:

$$
c_{p_{h}}=\left(c_{p_{a}}+c_{p_{w}} S H_{C i}\right) /\left(1+S H_{C i}\right)
$$

For dry air $R_{a}=0.287 \mathrm{~kJ} / \mathrm{kg} \mathrm{K}$, and for water vapor it is $R_{w}=0.462 \mathrm{~kJ} / \mathrm{kg} \mathrm{K}$. The 
specific heat capacities however are modeled as functions of temperature by polynomials [147]. From Equations (3.5) to (3.7), it is understood that $S H_{C i}$ affects the compressor performance by its effect on the corrected shaft speed $N_{C_{c o r}}$. Using the isentropic efficiency and the pressure ratio of the compressor from Equation (3.3), the temperature of the compressed humid air at the outlet of the compressor is

$$
T_{C o}=T_{C i}\left(\left(P R_{C}\left(\gamma_{h}-1\right) / \gamma_{h}-1\right) / \eta_{C}+1\right) .
$$

The mass flow in the compressor is calculated by its dimensionless group formula:

$$
W_{C}=W_{C_{c o r}}\left(P_{C i} \sqrt{T_{s t d} \gamma_{h} R_{a}}\right) /\left(P_{s t d} \sqrt{T_{C i} \gamma_{a} R_{h}}\right),
$$

where $P_{\text {std }}=101.325 \mathrm{kPa}$ is the standard atmosphere pressure. As a result, the required power of the compressor can be found by:

$$
P W_{C}=W_{C} c_{p_{h}}\left(T_{C o}-T_{C i}\right) .
$$

It should be noted that both $\gamma_{h}$ and $c_{p_{h}}$ are temperature dependent, and the average of their respective values in the range of $T_{C i}$ and $T_{C o}$ should be utilized in Equations (3.8) and (3.10). Equations (3.3) through (3.10) accomplish the model of the compressor when operating with humid air.

Combustion Chamber: For cooling the turbine nozzle and blades, a fraction of the compressed air $\left(W_{b}\right)$ bypasses the combustion chamber. The rest of the compressed air undergoes the combustion process with the injected fuel, which has a mass ratio of $f=W_{F} /\left(W_{C}-W_{b}\right)$. From the first law of thermodynamics, the temperature of the combustion product can be found as:

$$
T_{C h o}=f\left(c_{p_{F}}\left(T_{F}-T_{C o}\right)+L C V\right) /\left((1+f) c_{p_{P}}\right)+T_{C o},
$$

where $L C V$ is the lower calorific value of the fuel, which is $47000 \mathrm{~kJ} / \mathrm{kg}$ for natural gas in this study and,

$$
c_{p_{P}}=c_{p_{h}}+f /(1+f) \theta_{c_{p}}
$$

is the specific heat capacity of the combustion product with humid air. The specific heat capacities $c_{p_{F}}$ and $c_{p_{h}}$, and the parameter $\theta_{c_{p}}$ are temperature dependent [136]. 
Therefore, the average of respective $c_{p_{F}}$ in the range of $T_{F}$ and $T_{C o}$ should be used in Equation (3.11). Likewise, the average of respective $c_{p_{h}}$ and $\theta_{c_{p}}$ in the range of $T_{C o}$ and $\mathrm{T}_{\text {Cho }}$ should be used in Equation (3.12). As a result, Equation (11) needs to be solved through iterations.

Turbine: The bleed air returns to the turbine for cooling the nozzle and the first stage blades. The effective temperature at the turbine inlet is therefore:

$$
T_{T i}=\left(W_{C h} c_{p_{P}} T_{C h o}+W_{b} c_{p_{h}} T_{C o}\right) /\left(W_{C h} c_{p_{P}}+W_{b} c_{p_{h}}\right) .
$$

where $W_{C h}$ is the mass flow of the combustion products flowing out of the combustion chamber, which equals to:

$$
W_{C h}=W_{C}-W_{b}+W_{F} .
$$

The turbine map provides the rescaled corrected mass flow and the isentropic efficiency of the turbine versus the corrected shaft speed and the pressure ratio as shown in Figure 3.1 .

$$
\left[N_{T_{c o r}} / N_{D} \times W_{T_{c o r}}, \eta_{T}\right]=\operatorname{Map}_{T}\left(N_{T_{c o r}}, P R_{T}\right)
$$

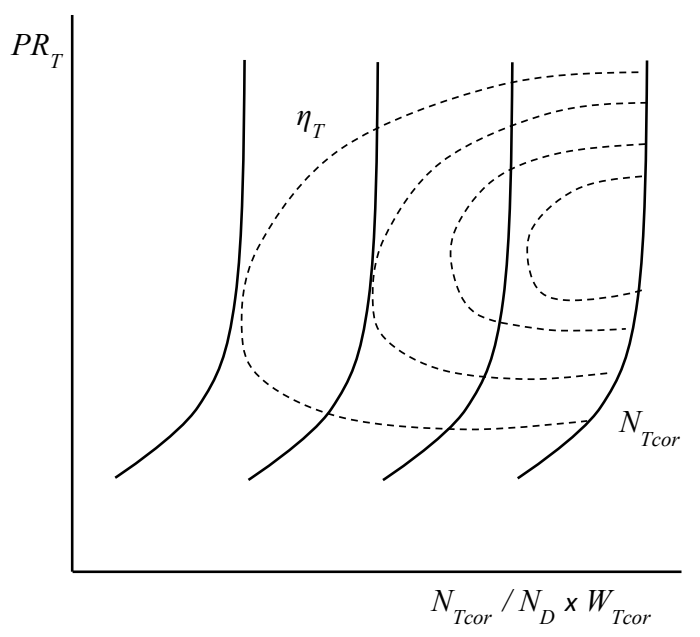

Figure 3.1. Schematic dimensionless turbine performance map.

Besides the temperature, the thermodynamic properties of the working fluid have to be taken into account to correct the turbine shaft speed through its dimensionless group 
formula:

$$
N_{T_{c o r}}=N \sqrt{\gamma_{a_{D}} R_{a_{D}} T_{T i_{D}}} / \sqrt{\gamma_{M i x} R_{M i x} T_{T i}},
$$

where $T_{T i_{D}}$ is the turbine inlet temperature at its design point, which is $1323 \mathrm{~K}$ for the GTE in this study. $R_{a_{D}}$ and $\gamma_{a_{D}}$ are the constant and the specific heat ratio of air at the design point temperature. $R_{M i x}$ and $\gamma_{M i x}$ are the constant and the specific heat ratio of the mixture of the combustion product with the cooling air, and can be found through the weighted averaging:

$$
\begin{aligned}
& R_{\text {Mix }}=\left(W_{C h} R_{P}+W_{b} R_{h}\right) /\left(W_{C h}+W_{b}\right), \\
& c_{p_{M i x}}=\left(W_{C h} c_{p_{P}}+W_{b} c_{p_{h}}\right) /\left(W_{C h}+W_{b}\right),
\end{aligned}
$$

and

$$
\gamma_{\text {Mix }}=c_{p_{\text {Mix }}} /\left(c_{p_{\text {Mix }}}-R_{\text {Mix }}\right),
$$

The gas constant for the combustion product in dry air condition is provided in [136]:

$$
R_{P_{d r y}}=R_{a}(1+f(6.1848 \bar{H}-\bar{C})) /(1+f),
$$

where $\bar{H}$ and $\bar{C}$ are the weight ratios of hydrogen and carbon in the fuel, respectively. By adding the effect of the humidity through weighted averaging, the gas constant of the combustion product in humid air condition is:

$$
R_{P}=\left(R_{P_{d r y}}(1+f)+R_{w} S H_{C i}\right) /\left(1+f+S H_{C i}\right),
$$

Equations (3.17) through (3.21) find the corrected shaft speed for the turbine map. The pressure ratio of the turbine equals to the pressure ratio of the compressor, reduced by the losses in the gas path:

$$
P R_{T}=P R_{C} P R_{L}
$$

The turbine map outputs are the corrected and rescaled mass flow and the isentropic efficiency. The mass flow of the turbine is therefore calculated by:

$$
W_{T}=W_{T_{c o r}}\left(P_{T i} N_{D} \sqrt{T_{\text {std }} \gamma_{\text {Mix }} R_{a}}\right) /\left(P_{s t d} N_{T_{c o r}} \sqrt{T_{T i} \gamma_{a} R_{\text {Mix }}}\right),
$$

where $N_{D}$ is the GTE shaft speed at the design point, which equals 16500 RPM for the 
GTE in this study, and $P_{T i}=P_{C i} P R_{C} P R_{C h}$ is the inlet pressure of the turbine. The turbine map in Equation (3.15) provides the isentropic efficiency of the expansion, and consequently, the $E G T$ and the power of the turbine can be calculated:

$$
\begin{aligned}
& E G T_{M}=T_{T i}\left(\eta_{T}\left(P R_{T}\left(1-\gamma_{M i x}\right) / \gamma_{M i x}-1\right)+1\right), \\
& P W_{T}=W_{T} c_{p_{M i x}}\left(T_{T i}-E G T_{M}\right) .
\end{aligned}
$$

The heat capacity ratio and the specific heat capacity of the exhaust gas are temperature dependent and should be set to the average of their respective values in the range of $T_{T i}$ and $E G T_{M}$.

The overall system: The models of the GTE sub-assemblies must be compatible at a time to build an integral model for the overall system. The principle of mass conservation requires $W_{T}=W_{C}+W_{F}$, which should be achieved by readjusting $\beta$ value in Equation (3.3). When the system of the equations from Equations (3.3) to (3.25) becomes consistent, the output power of the GTE can be found by:

$$
P W_{G T E_{M}}=P W_{T}-P W_{C}-P W_{L},
$$

where $P W_{L}$ represents all the internal losses, which is usually assumed one percent of the compressor power [133].

Equations (3.3) through (3.26) model the gas path parameters of an operating single shaft GTE. In the closed form, the power and the EGT can be presented as the following:

$$
\begin{aligned}
& P W_{G T E_{M}}=P W_{G T E_{M}}\left(T_{C i}, P_{C i}, S H_{C i}, N, f\right), \\
& E G T_{M}=E G T_{M}\left(T_{C i}, P_{C i}, S H_{C i}, N, f\right) .
\end{aligned}
$$

Fuel to air mass ratio $(f)$ is not available in the measurements of many turbines, including this study. In the next sections, it is explained how to handle the modeling without measurements on this parameter.

Equations (3.27) and (3.28) are the generalized form of Equations (3.1) and (3.2) considering the specific humidity of air, and they are expected to improve the modeling uncertainties in the primary model, caused by the humidity variations. Figure 3.2 depicts the modeling process flow at both the sub-assembly level and the system level. 


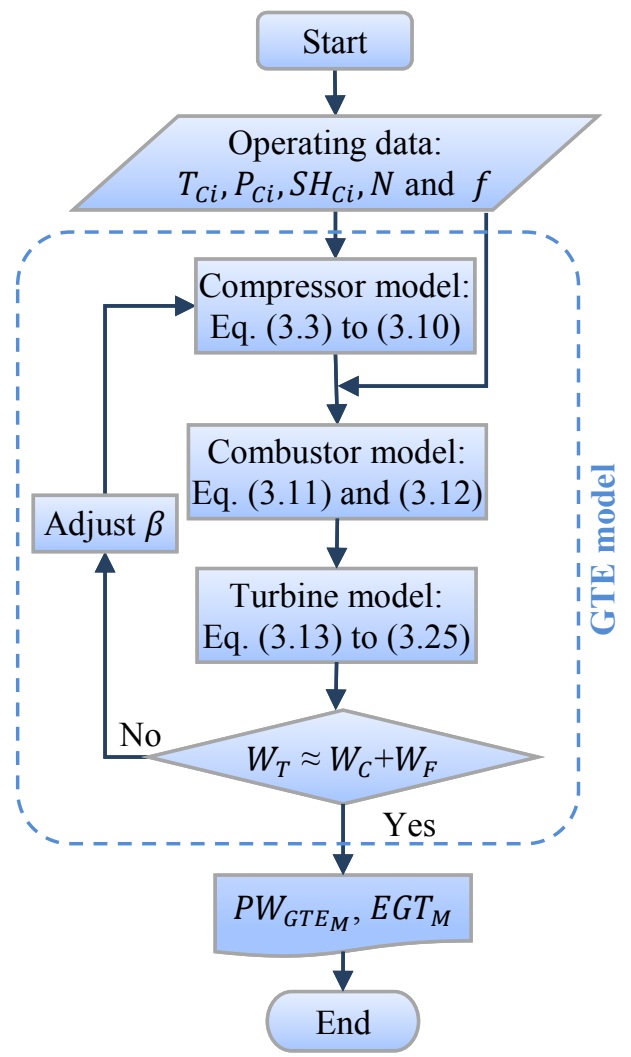

Figure 3.2. Model of the GTE with air humidity, given the compressor and the turbine maps.

\subsection{Inlet Air Cooling}

The humidity content in the GTE intake air may come from the natural humidity of the ambient or may be added by the inlet air cooling system between the air filter and the engine. In either case, all of the moisture content is taken into the engine with the intake flow and eventually undergoes the thermodynamic cycle, which was elaborated in the previous section. Therefore, the final specific humidity $S H_{C i}$ is needed for modeling the performance of the GTE. In most cases, including the case in this study, the temperature of the engine inlet air is the only property available from this section, while the flow of the cooling water is unknown. Therefore, given the ambient temperature, pressure and relative humidity, we should identify the following relation for the specific humidity:

$$
S H_{C i}=S H_{C i}\left(T_{a m}, P_{a m}, \phi_{a m}, T_{C i}\right) .
$$

Given the relative humidity of the ambient air $\left(\phi_{a m}\right)$, the specific humidity is found by 
[148]:

$$
S H_{a m}=0.622 \phi_{a m} P_{w}^{*} /\left(P_{a m}-\phi_{a m} P_{w}^{*}\right),
$$

where $P_{w}^{*}$ is the saturation vapor pressure at temperature $T_{a m}$, suggested by an empirical equations by Buck [149]. The inlet air cooler does not tangibly change the pressure, therefore we assume $P_{C i} \approx P_{a m}$. The cooling water comes from an outdoor filtering chamber and it is assumed to have the same temperature of the ambient. From the first law of thermodynamic and mass conservation principle, the specific humidity after the cooler is:

$$
S H_{C i}=\left(c_{p_{a}}\left(T_{a m}-T_{C i}\right)+S H_{a m}\left(h_{g_{a m}}-h_{f_{a m}}\right)\right) /\left(h_{g_{C i}}-h_{f_{a m}}\right),
$$

where $h_{f}$ and $h_{g}$ represent the specific enthalpies of saturated liquid and vapor water in the respective temperatures.

As a numerical example, in an ambient condition with $T_{a m}=20^{\circ} \mathrm{C}, P_{a m}=101 \mathrm{kPa}$ and $\phi_{a m}=50 \%$, if the temperature after the cooler drops to $T_{C i}=15^{\circ} \mathrm{C}$, the respective specific humidity after the cooler reaches to $S H_{\mathrm{Ci}}=0.0094$ that corresponds to $\phi_{C i}=88 \%$.

Equations (3.30) and (3.31) together provide the specific humidity of air at the inlet of the machine, which is required for the enhanced modeling of the GTE with humid air, as specified in Equations (3.27) and (3.28). It should be noted that except for Equations (3.14) and (3.26), which refer to the laws of conservation of mass and energy, all other equations are approximations within the acceptable ranges of modeling accuracies.

\subsection{Performance Evaluation}

In Chapter 2, two physics-based performance indicators, i.e., excess heat and power deficit were introduced for GTEs performance monitoring. An extension on the concept of excess heat indicator is to take into account the extra enthalpy that is wasted through the exhaust due to performance deterioration of the GTE [25]. We define Excess Heat Ratio $(E H)$ as the normalized value of the extra enthalpy discarded from a GTE exhaust, compared to the modeled as new conditions, i.e., the EGT, predicted by a model that is calibrated with the newly commissioned GTE conditions. 


$$
\begin{aligned}
E H & =\left(\dot{H}_{T o}-\dot{H}_{T o_{M}}\right) / P W_{G T E_{D}} \\
& =W_{T} c_{p_{M i x}}\left(E G T-E G T_{M}\right) / P W_{G T E_{D}},
\end{aligned}
$$

where $P W_{G T E_{D}}$ is the power of the GTE at the design point and $c_{p_{\text {Mix }}}$ is the average specific heat capacity of the exhaust gas in the range of the measured and the modeled temperatures. $E G T_{M}$ represents the predicted exhaust gas temperature, given the modeled power of the GTE equals the measured power, i.e., $P W_{G T E_{M}}=P W_{G T E}$. By this assumption, Equation (3.27) can be solved for $f$, and $E G T_{M}$ will then be found using Equation (2.28).

Similar to excess heat indicator, $E H$ is robust to the variations of the ambient conditions and the operating scenarios even without modeling the humidity effects [25]. In this section, $E H$ is recalculated, using the developed enhanced GTE model considering

the humidity effects. Because the new model takes into account the effects of the humidity, it is expected to mitigate the modeling uncertainty and consequently reduce fluctuations in the computed performance indicator, compared to the case where the primary model is employed.

For the GTE in the study, figure 3.3 shows the ambient temperature, the relative humidity, and the specific humidity at the engine inlet throughout the operating period. The seasons are marked in Figure 3.3(c) where we see the moisture content of the air increases in the summers and decreases in the winters. There are two reasons for such phenomena: 1) the higher the air temperature, the more capacity it has to hold the moisture; 2) the cooling system adds more moisture to the intake air during the warm summer days. The moisture content reaches to around $2 \%$ of the intake flow during some days, which alters the thermodynamic properties of the working fluid and affects the GTE performance, and it needs to be considered by the enhanced model.

\subsubsection{EH Calculation Model}

$E H$ is defined based on the difference between the actual exhaust losses and the modeled exhaust losses, calibrated with the brand-new design parameters. If the model can accurately predict the gas path parameters, any rise in $E H$ will be attributed to the changes in the presumed design parameters, which take place due to various degradation 
mechanisms. Any inaccuracy in the thermodynamic model affects the corresponding $E H$ value and will be interpreted as a performance change, while it is not caused by the actual degradation of the machine. Consequently, the modeling uncertainty leads to the growth of the noise in $E H$ signal. Figure 3.4(a) shows $E H_{a}$, which uses the primary model of the GTE in [25]. $E H_{h}$ is depicted in Figure 3.4(b), for which the enhanced GTE model is employed. The difference, i.e., $\Delta E H=E H_{h}-E H_{a}$, which is shown in Figure 3.4(c) corresponds to the noise in $E H_{a}$, due to the humidity-related modeling uncertainty. Figure 3.4(c) clearly shows that the absolute $\Delta E H$ increases when there is more moisture content in the intake air, which is the dominant case during the summers. Although the moisture content of the air is a positive value, we observe that its effect is not always in a single direction. In other words, $\triangle E H$ takes both positive and negative values, depending on the other parameters in the operating conditions. To find a quantitative relation between the humidity and $\Delta E H$, the normalized $\Delta E H$ with respect to the actual index value to be studied.

$$
\Delta E H_{n}=\left(E H_{h}-E H_{a}\right) / E H_{h}
$$

Local variation of $\Delta E H_{n}$ is a measure of the uncertainty in the $E H$ calculation model. Figure 3.5 depicts the standard deviation and the mean value of $\Delta E H_{n}$ within a onemonth moving window throughout the operating period, i.e., at each point, the plot shows the standard deviation and the mean of $\Delta E H_{n}$ signal in an interval from half a month before to half a month after the current point in time. The window size is chosen such that seasonal variations can be seen in $\Delta E H_{n}$ signal, while its local spikes are removed. As it is shown in Figure 3.5(a), the humidity-related uncertainty alters from $0.13 \%$ up to $1.4 \%$, while the average $\Delta E H_{n}$ varies between $-2 \%$ and $0.5 \%$. Although the values may seem small at first glance, their importance should be noted considering the following points: 1) a cyclic variation is clearly observed in the standard deviation plot, which is in compliance with the variations of the specific humidity, shown in Figure 3.3(c). It is observed that whenever the specific humidity rises, the uncertainty of the calculation model of $E H_{a}$ increases; 2) on average, with the rise of humidity, the expected $\Delta E H_{n}$ decreases; and 3) even a small improvement in the performance indicator is a useful practice, when there is no information available about the other sources of uncertainties. 
(a)

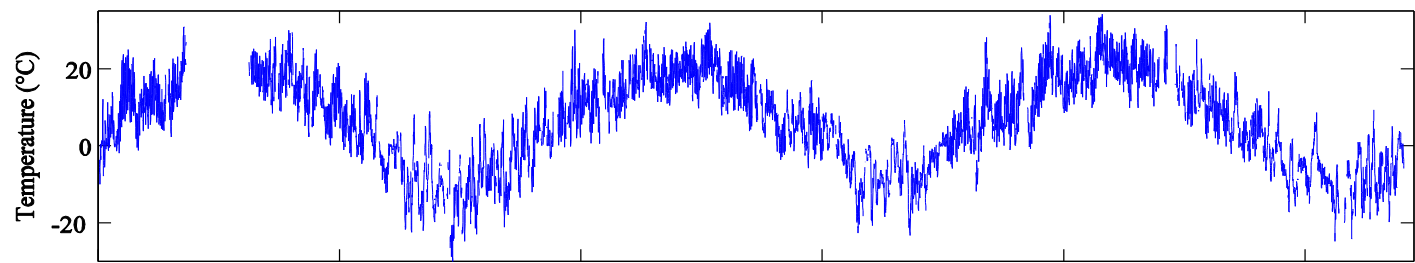

(b)

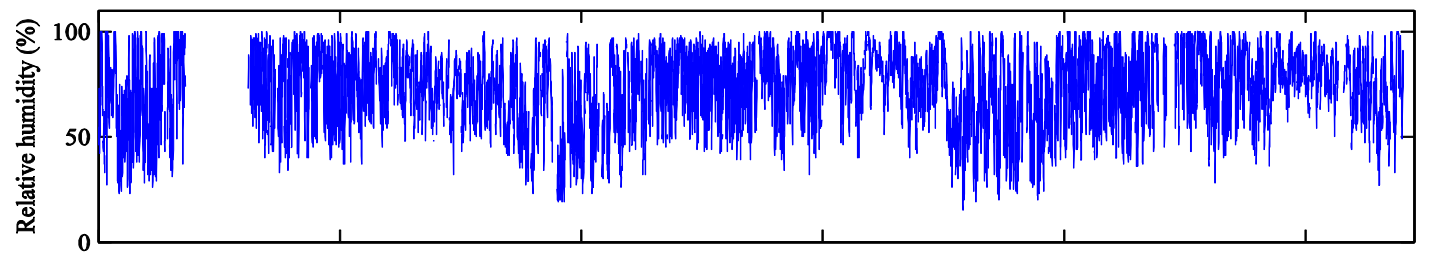

(c)

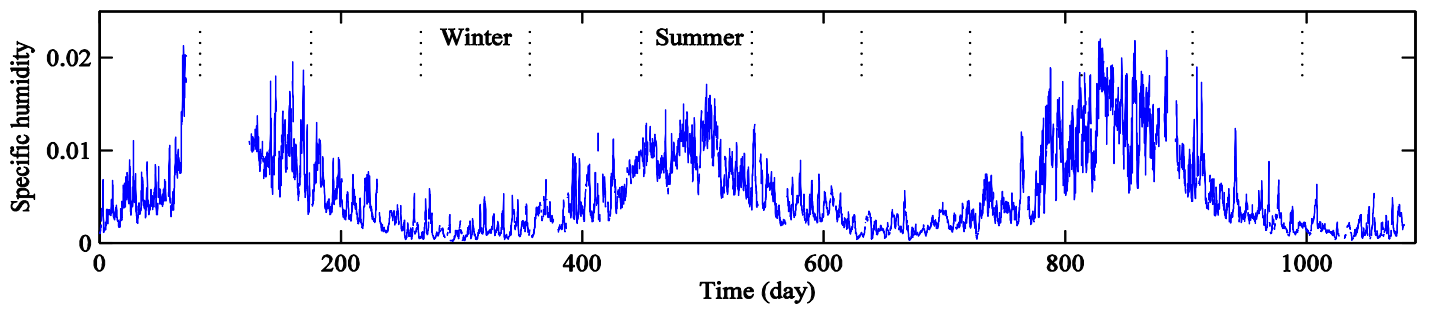

Figure 3.3. Variations of the ambient condition and the moisture content: (a) ambient temperature, (b) ambient relative humidity, and (c) specific humidity at the GTE inlet.

(a)

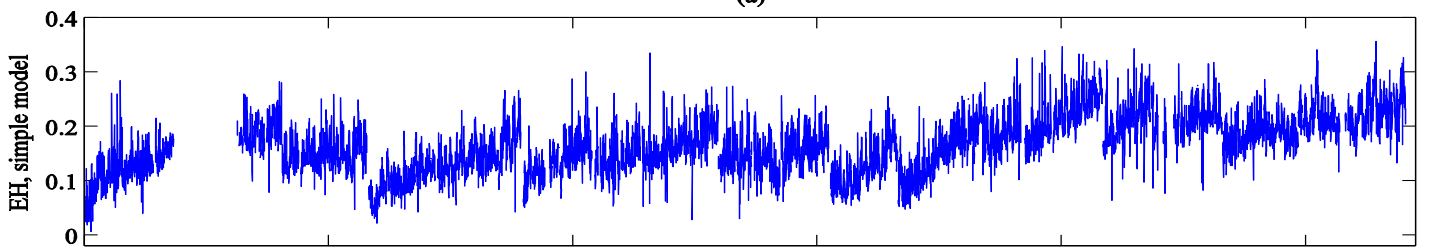

(b)
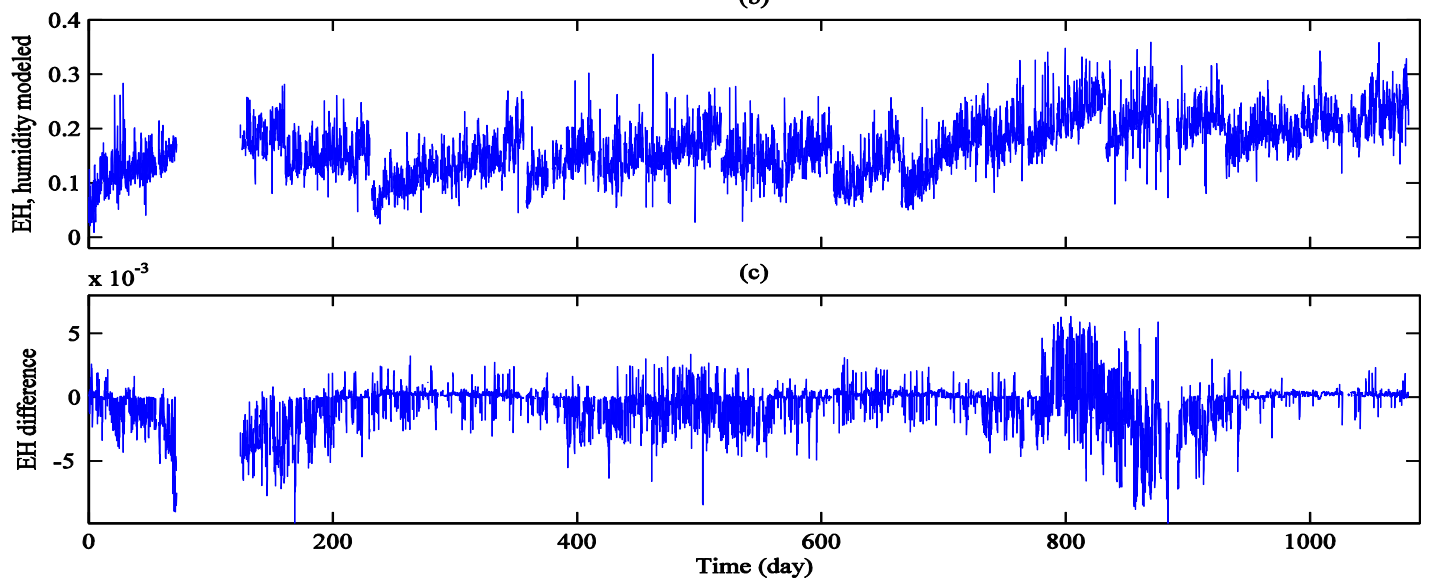

Figure 3.4. Variations of the performance indicator: (a) $E H_{a}$, (b) $E H_{h}$, and (c) the difference: $\Delta E H$. 

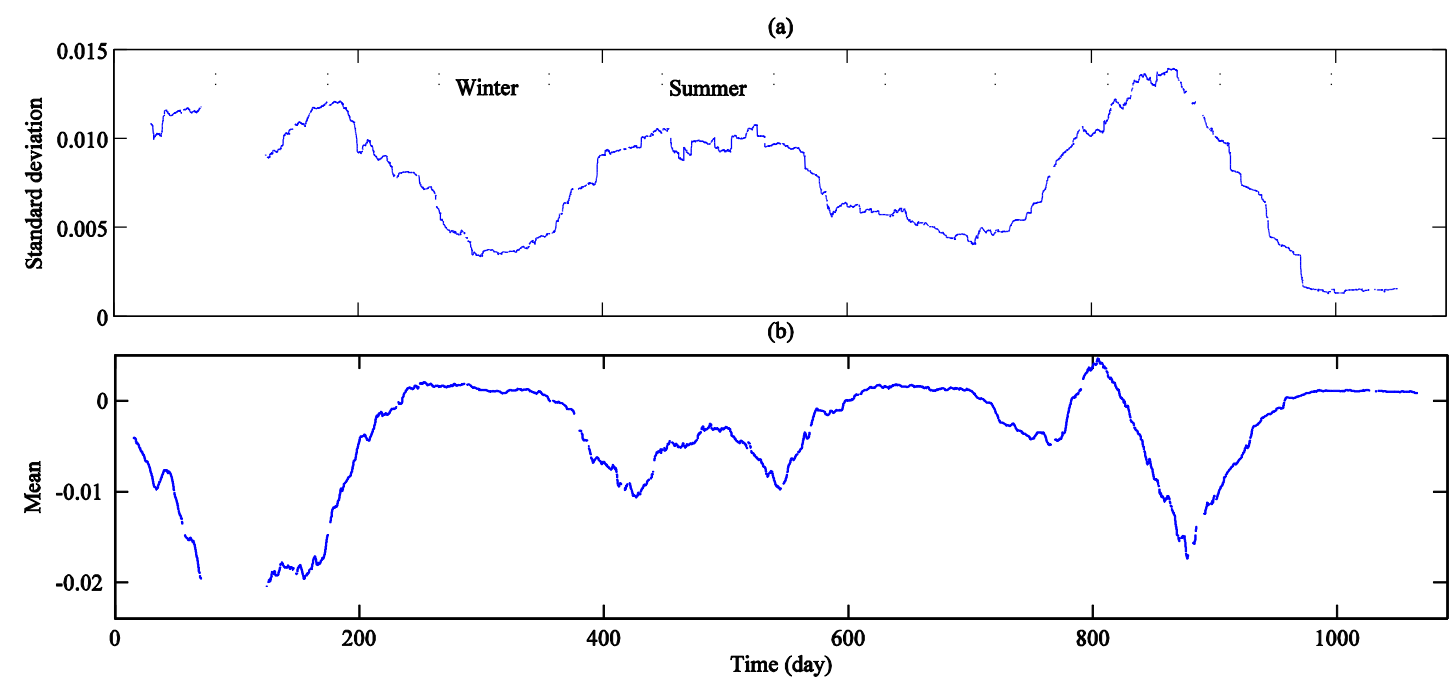

Figure 3.5. Variations of $\Delta E H_{n}$ statistical characteristics: (a) standard deviation, and (b) mean value.

\subsection{2 $\quad$ EH Prediction Model}

There are two distinct trends in the variations of $E H$ index as shown in Figure 3.4(a) and (b). The plot shows that $E H$ varies with a saw-tooth pattern, which comprises a gradual growth and an abrupt drop in each segment, corresponding to two consecutive washes [141]. In the long-term, an overall ascending trend is observed in $E H$ variations. Trend modeling provides a means to predict the future performance of a GTE. Modeling the trends with the linear regression was discussed in Chapter 2 and in [25]. In this part, double asymptotic exponential function is used for trend modeling and the accuracies of the established models on $E H_{a}$ and $E H_{h}$ signals are investigated and compared. To model the short-term performance deterioration between the consecutive compressor washes, the general form of the mentioned function is:

$$
E H_{M}=\alpha_{1}\left(1-e^{\alpha_{2} t}\right)+\alpha_{3}\left(1-e^{\alpha_{4} t}\right) .
$$

The model is fitted to $E H_{a}$ and $E H_{h}$ signals in all segments and the corresponding coefficients of the function are found. Table 3.1 provides the coefficients of the fitted curve in segment 7 as an example, and Figure 3.6 shows $E H_{h}$ signal and the fitted curve in segment 9 . The prediction modeling error at a single point is: 


$$
\varepsilon=E H-E H_{M}
$$

Similar to the section 3.4.1, the local standard deviation of the modeling error within a one-month window is taken as the measure of the prediction model uncertainty. To compare the two cases $\left(E H_{a}\right.$ and $\left.E H_{h}\right)$, the local standard deviation of each signal is found, and the corresponding ratio is calculated as per Equation (3.36).

$$
S T D_{\text {ratio }}=\operatorname{STD}\left(\varepsilon_{a}\right) / S T D\left(\varepsilon_{h}\right) .
$$

Table 3.1. Function coefficients in segment 7

\begin{tabular}{|c|c|c|c|c|}
\hline Index & $\alpha_{1}$ & $\alpha_{2}$ & $\alpha_{3}$ & $\alpha_{4}$ \\
\hline \hline$E H_{a}$ & $-1.76 \mathrm{E}-01$ & $-6.95 \mathrm{E}+03$ & $-1.88 \mathrm{E}-01$ & $2.15 \mathrm{E}-03$ \\
$E H_{h}$ & $-2.10 \mathrm{E}-01$ & $-2.09 \mathrm{E}+04$ & $-2.89 \mathrm{E}-01$ & $1.72 \mathrm{E}-03$ \\
\hline
\end{tabular}

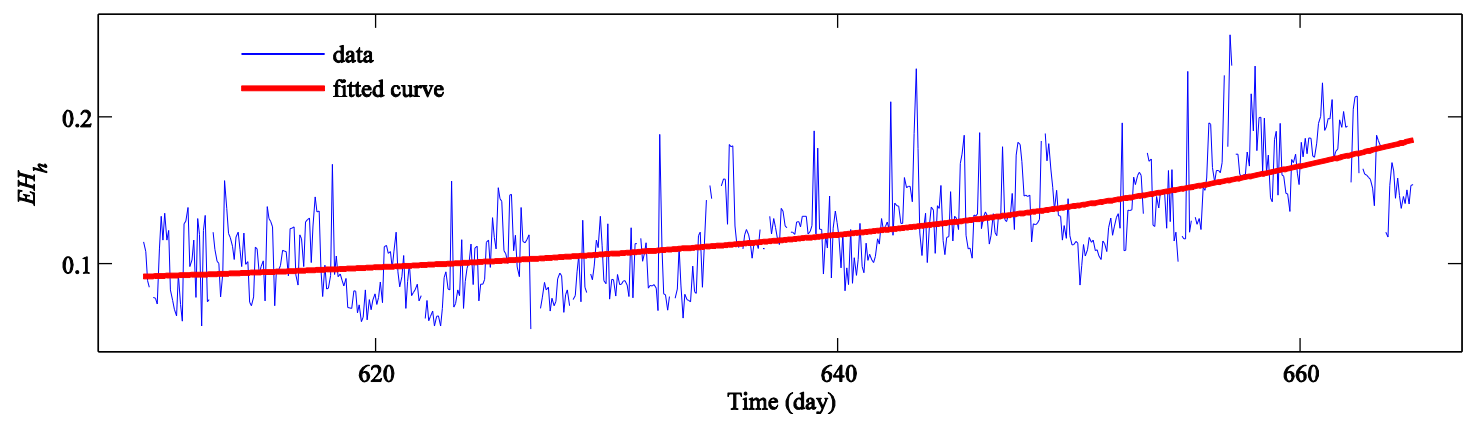

Figure 3.6. $E H_{h}$ signal and the fitted model in segment 9.

Figure 3.7 shows this ratio throughout the operating period. As the plot shows, the modeling uncertainty of the fitted curve on $E H_{a}$ signal is slightly more than that of $E H_{h}$ throughout almost the entire operating period. This means, variations of $E H_{h}$ signal with respect to the fitted model are smaller, compared to $E H_{a}$ index, i.e., $E H_{h}$ is less noisy than $E H_{a}$. As a result, when the humidity effect is taken into account, the local variations of the performance indicator is mitigated and the performance can be predicted with less uncertainty. 


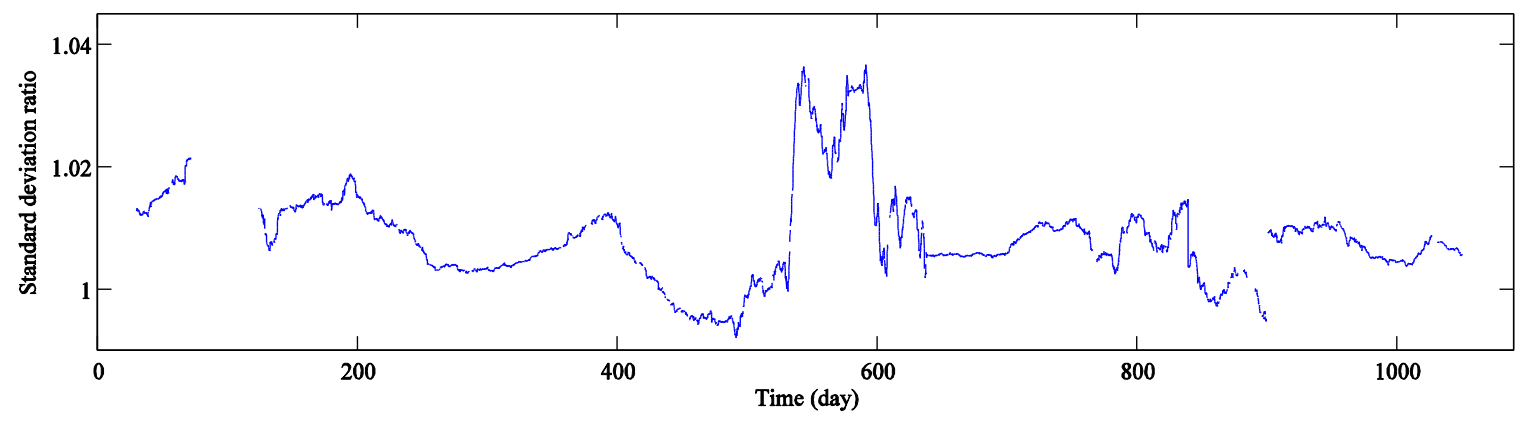

Figure 3.7. Standard deviation ratio for the modeling errors.

\subsection{Summary}

In this chapter, an enhanced thermodynamic model was developed for a single shaft GTE considering the humidity content of the intake air. The model uses the ambient condition data and the basic operating parameters from the operating system of the GTE, and predicts the gas path parameters. A separate thermodynamic model was developed for the inlet air cooler to estimate the moisture content of the GTE intake air. A unified physics-based performance indicator, i.e. excess heat ratio was defined as the normalized difference between the actual enthalpy of the exhaust gas, and the expected enthalpy of the exhaust gas, modeled for the brand-new GTE condition. The proposed indicator was calculated using two GTE models, i.e., the primary model and the enhanced model, and the resulting indicators were compared from two aspects: 1) the absolute values of the performance indicators from the primary and the enhanced models were compared and the point-to-point difference was found at each time step. The result shows that there is a clear correlation between the local variation of the mentioned point-to-point differences and the moisture content in the ambient air. It indicates that inclusion of the humidity effects in GTE modeling provides more accuracy to the performance indicator, especially when the moisture content of the intake air increases; 2) the double asymptotic exponential function was used to fit prediction models on each indicator signal, and the modeling error was calculated. The results suggest a better predictability with the performance indicator using the enhanced GTE model. 


\section{Chapter 4: A Framework with Nonlinear System Model and Nonparametric Noise for Gas Turbine Degradation State Estimation}

\subsection{Overview}

Considering the complexity of the gas path thermodynamic models, degradation state estimation from the limited measurements of the GTE operating system is a great challenge. In this chapter, a model-data fusion framework is developed to sequentially use the operational measurement of a GTE and estimate loss of efficiency in the turbine section as the internal degradation symptom. Given the nonlinear GTE model and nonparametric measurement noise, a framework with regularized auxiliary particle filter (RAPF) is developed to manage non-uniform measurement sequences and redundant measurements, and the results are compared with another variant of particle filter (PF).

The developed framework applies the RAPF technique for GTE degradation state estimation for the first time. It is also new in that: there is no need for additional instrumentation, and the degradation state is estimated only from the measurements of the GTE operating system. This framework extends the RAPF technique to non-uniform time sequences, where the measurement noise has an unknown distribution.

\subsection{Development of the Performance Indicator}

The GTEs typically monitor a set of the operating parameters through their control console that are logged either manually or automatically on a regular basis. Such data can be utilized for gas path analysis, using thermodynamic models, and can provide valuable information about the performance of the GTE.

\subsubsection{Thermodynamic Model of the GTE for Performance Monitoring}

In Chapter 3 a comprehensive thermodynamic model for single shaft GTEs was developed. To predict the internal parameters of the GTE, the model was set up such that it only needs the external measurements on the GTE operating data that are readily available, i.e., intake air temperature $\left(T_{C i}\right)$, pressure $\left(P_{C i}\right)$ and relative humidity $(\phi)$, shaft 
speed $(N)$, power $(P W)$ and exhaust gas temperature $(E G T)$. Once the model is calibrated with the design parameters $(D P)$ of the newly installed condition, it will simulate the GTE operation for similar conditions. Namely, the model predicts the power and the exhaust gas temperature:

$$
\begin{aligned}
& P W_{G T E_{M}}=P W_{G T E_{M}}\left(T_{C i}, P_{C i}, \phi, N, W_{F}, D P\right), \\
& E G T_{M}=E G T_{M}\left(T_{C i}, P_{C i}, \phi, N, W_{F}, D P\right),
\end{aligned}
$$

where $W_{F}$ is the fuel mass flow. By eliminating $W_{F}$ from the equations and fixing the modeled exhaust gas temperature to the measured one, the power appears as a function of the available operating parameters:

$$
P W_{G T E_{M}}=P W_{G T E_{M}}\left(T_{C i}, P_{C i}, \phi, N, E G T, D P\right) .
$$

Given the lower calorific value of the fuel $(L C V)$, the predicted thermal efficiency of the GTE can be calculated:

$$
\eta_{G T E_{M}}=P W_{G T E_{M}} /\left(W_{F_{M}} L C V\right) .
$$

The actual thermal efficiency is more difficult to estimate, because the fuel mass flow is not directly measured. By fixing the EGT in the model, the actual thermal loss of the exhaust gas is well estimated by the model. Therefore, using the first law of thermodynamics, the model error to find the fuel energy is only attributed to the mismatch of the predicted and the actual power:

$$
\left(W_{F}-W_{F_{M}}\right) L C V=P W-P W_{G T E_{M}} .
$$

Consequently, the actual thermal efficiency of the GTE is:

$$
\eta_{G T E}=P W /\left(\left(W_{F_{M}} L C V\right)+P W-P W_{G T E_{M}}\right) .
$$

In practice, the difference between the predicted parameters and the actual measurements usually grows due to internal degradation of the GTE components, and provides a measure for the deterioration in the GTE performance. We developed and tested a number of physics-based performance indicators using the difference between the predicted and the measured parameters. Eventually, the "Efficiency Ratio" $(E R)$ was selected as the optimum choice for performance monitoring. $E R$ is defined the ratio 
between the actual thermal efficiency $\left(\eta_{G T E}\right)$ and the predicted thermal efficiency $\left(\eta_{G T E_{M}}\right)$, modeled in the same operating conditions:

$$
E R=\eta_{G T E} / \eta_{G T E_{M}} .
$$

Since the model takes into account the operating conditions, any reduction in $E R$ value can be attributed to the structural degradation of the internal components that is yet to be detected through diagnostics tools. Figure 4.1 illustrates the process flow, though which the performance indicator is calculated from the operating data.

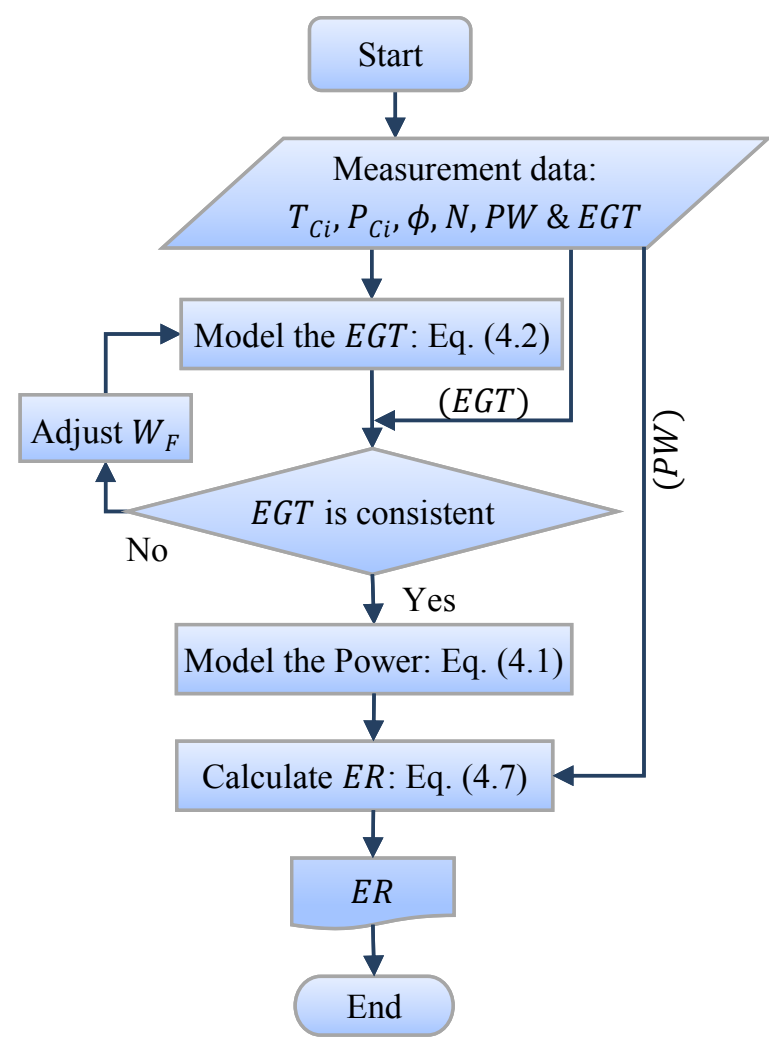

Figure 4.1. Process flow to calculate $E R$ indicator.

The performance of the GTEs deteriorates by time due to different degradation mechanisms of the components. The degradations are categorized in two different types: short-term degradation and long-term degradation. The former is mostly caused by fouling and congestion of the particulate matters on the gas path components. In 
particular, the blades and the vanes of the first stages of the compressor are affected more by the fouling. The short-term degradation has a rather quick rate; however, it is fortunately reversible through appropriate washing and cleaning services, which retrieve the GTE performance. Long-term degradation of the GTE is a term, used to describe the slow and mostly non-recoverable types of the degradations, caused by wear and tear mechanisms in the GTE components. The parts in hot section of the GTE are more vulnerable to this type of structural degradation.

Both types of the degradation take place simultaneously and cause overall deterioration in the GTE performance, and it is difficult to isolate them and find the root cause of the deterioration. One way to separate the individual contribution of each type of degradation is through analyzing the trend of a performance indicator. For this purpose, the variations of the performance indicator are segmented according to the intervals in which the gradual changes followed by rapid retrievals are observed. One can assume that during the first days after washes, the compressor is still clean and free from the fouling effect. The performance indicator will consequently display the performance deterioration entirely caused by the non-recoverable structural degradations occurring during such periods.

To assess the effectiveness of the developed indicator and to observe the short-term and the long-term performance deteriorations, a set of data from the operating system of a single shaft simple-cycle industrial GTE has been utilized. In Figure 4.2 the variation of the proposed $E R$ indicator is shown, which is used to differentiate the short-term and the long-term performance deteriorations. Figure 4.2(b) shows the GTE performance quickly deteriorates in the short-term and then retrieves by the compressor wash, e.g., from 3864 to 5522 hours, $E R$ declines from 0.98 to 0.95 , and then abruptly climbs to about the same initial performance after the consequent wash. However, the overall performance gradually deteriorates about $10 \%$ during the whole working period as appears in Figure 4.2(a). There are some gaps in the performance plot, e.g., from hour 1724 to hour 2982, due to the GTE downtime. 
(a)

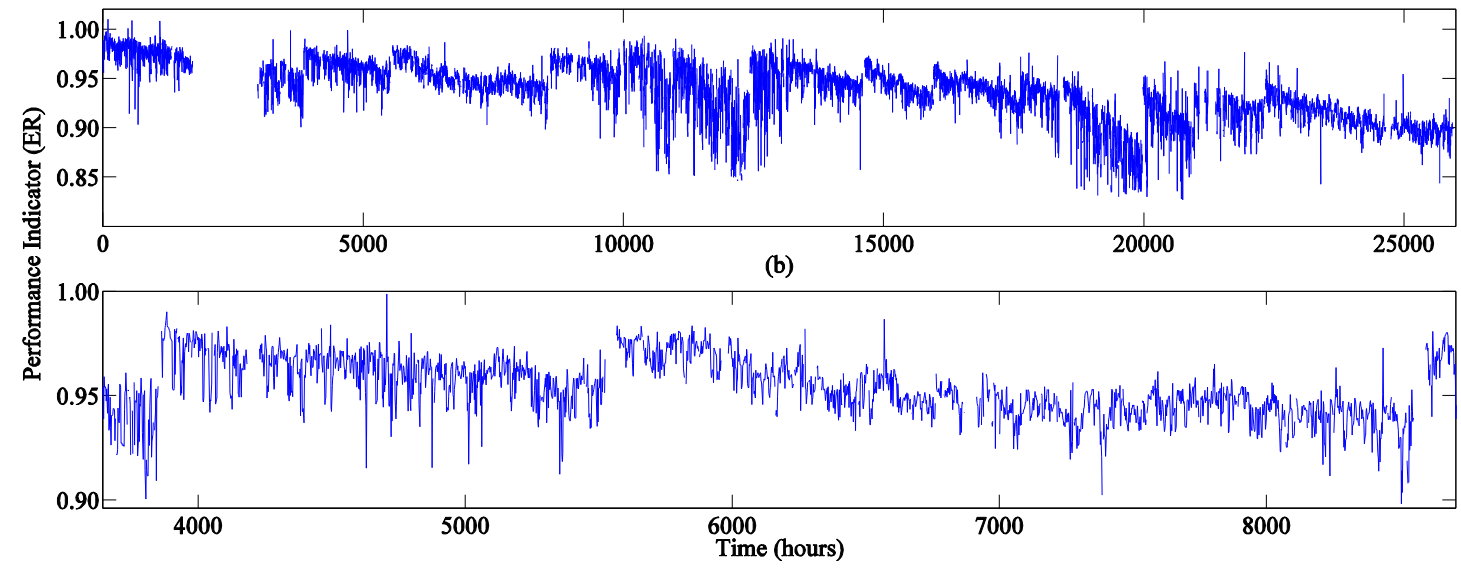

Figure 4.2. Performance deterioration due to the short-term and the long-term degradations: (a) overall performance variation, and (b) Selected segments depicting short-term performance variation.

\subsubsection{Turbine Degradation Symptom: the Internal State}

The structural degradation mechanisms in the turbine section of a GTE appear with different fault modes: 1) The surface roughness of the blades and the vanes increase due to erosion and corrosion phenomena; 2) The geometry of the profiles may change due to major material removal, cracking and bowing, and 3) The tip clearance of the blades with the stator and the vanes with the rotor can increase due to abrasion [138].

The degradation mechanisms result in two major symptoms in the turbine, i.e., the isentropic efficiency of the turbine declines, and the through flow capacity increases. While the former is the result of all the three fault modes, the latter is associated with loss of profile, especially in the trailing edge of a profile [4]. The loss of the isentropic efficiency is therefore a symptom that provides a more holistic indication of the overall degradation in the turbine section. This symptom cannot be directly measured and has to be indirectly estimated. The aim of this work is to identify the long-term degradation of the turbine through the inference from the GTE performance variations to the symptom of "loss of isentropic efficiency". Figure 4.3 shows the cause and the effect relation of the structural degradation and performance deterioration in the GTE and the inference process to identify the root cause. 


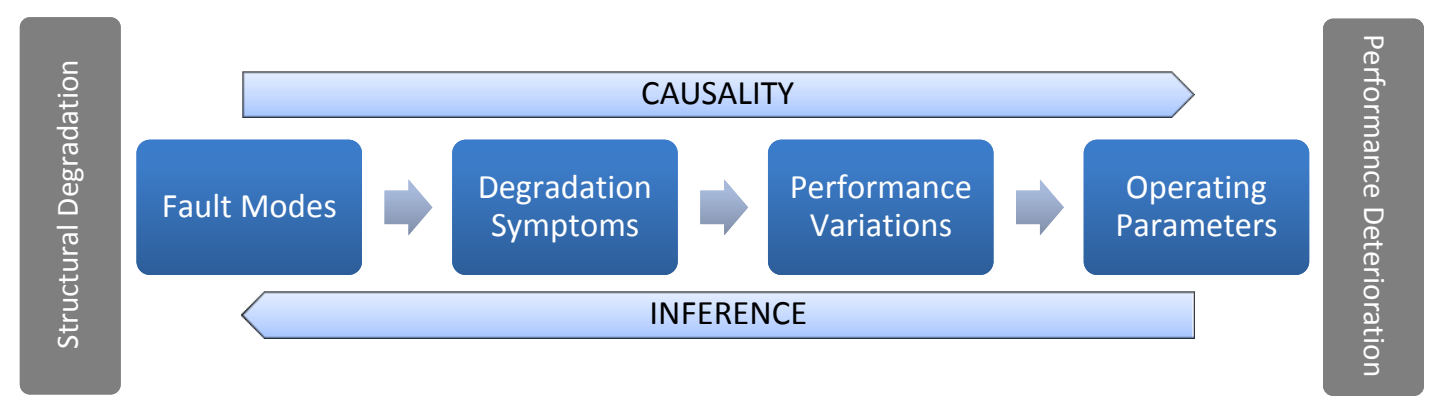

Figure 4.3. Forward causality and backward inference in the GTE degradation.

As stated in 4.2.1, the GTE performance in the early days after the compressor wash is not yet influenced by the fouling effects and can be used to estimate the long-term performance deterioration. On one hand, the scant data measured shortly after the washes do not provide a good statistical measure, and on the other hand, as the time window, during which the measurements are taken expands, the fouling effects decrease the quality of the data. After studying different bin sizes, we chose the data from the first five days after each wash, which comprises 60 readings. To avoid the effects of the outliers and to get representative values for each bin, the median of the samples in each bin is considered as a reliable value for the central tendency of the data [150]. Figure 4.4(a) shows the variations of the performance indicator, shortly after washes that represent the long-term performance deterioration.

The isentropic efficiency of the turbine extracted from the turbine map acts as a design parameter ( $\eta_{T} \in D P$ ) in the GTE model Equation (4.3). To model the effects of the turbine degradation, we seed the fault in the form of a correction factor $(1-\rho)$ to adjust the isentropic efficiency, where $\rho$ is the ratio of the isentropic efficiency loss in the turbine,

$$
\eta_{T_{c o r}}=(1-\rho) \eta_{T}
$$

such that the model results match the actual measurements, given the same ambient and operating conditions,

$$
\eta_{G T E}\left(:, \eta_{T_{c o r}}\right)=E R \eta_{G T E_{M}}\left(:, \eta_{T}\right) .
$$

There are different choices for the time-based model for degradation state prediction, 
including physical model-based methodology, knowledge based methodology, and datadriven methodology [114]. Similar to many reported degradation models, an exponential behavior for fault symptom evolution can be assumed [151],

$$
\rho_{M}(t)=\alpha_{1}\left(1-e^{\alpha_{2} t}\right)
$$

where $t$ is the time in hours, and the coefficients of the model can be found through the best fit to the GTE measurement records: $\alpha_{1}=-0.0285$ and $\alpha_{2}=0.0257 \times 10^{-3}$ $\left(\mathrm{hr}^{-1}\right)$. Figure 4.4(b) shows the factor of the efficiency loss, i.e. the seeded fault, required to fit the model to the actual performance, and the efficiency loss modeled by Equation (4.10). There is apparently some modeling error,

$$
\varepsilon=\rho-\rho_{M},
$$

which creates uncertainty in state prediction and should be managed appropriately. Table 4.1 provides the modeled state parameter and the error at each sequence.

(a)

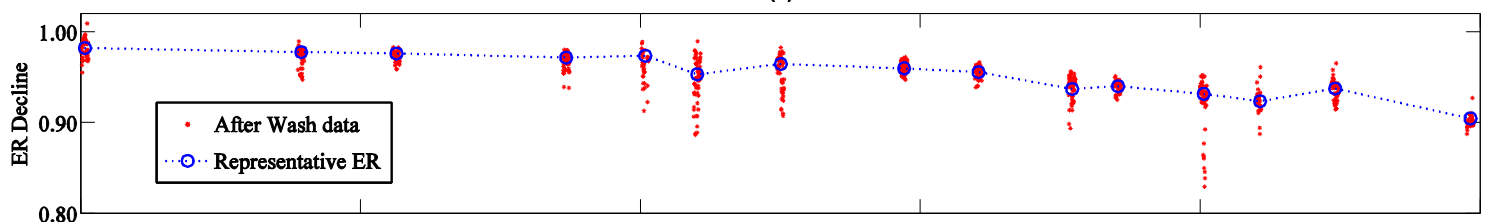

(b)

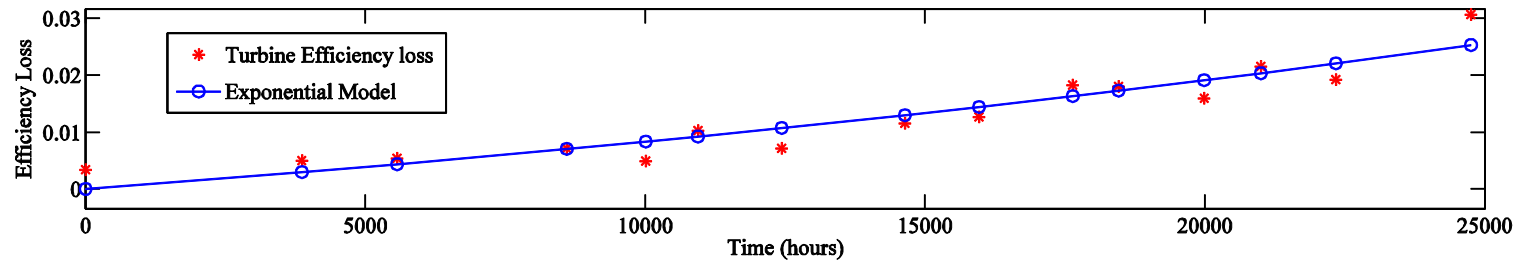

Figure 4.4. (a) Long-term performance deterioration: decline of $E R$ index, and (b) degradation of the turbine: ratio of the isentropic efficiency loss. 
Table 4.1. Efficiency loss and modeling error

\begin{tabular}{ccc}
\hline \hline Time $(\mathrm{h})$ & $\rho_{M}(t)(\%)$ & $\varepsilon(t)(\%)$ \\
\hline 2 & 0 & 0.335 \\
3864 & 0.297 & 0.202 \\
5566 & 0.437 & 0.099 \\
8598 & 0.703 & 0.014 \\
10010 & 0.833 & -0.346 \\
10940 & 0.922 & 0.097 \\
12436 & 1.070 & -0.354 \\
14638 & 1.297 & -0.147 \\
15964 & 1.441 & -0.180 \\
17638 & 1.629 & 0.191 \\
18458 & 1.724 & 0.077 \\
19988 & 1.907 & -0.315 \\
20998 & 2.032 & 0.119 \\
22340 & 2.203 & -0.283 \\
24754 & 2.525 & 0.532 \\
\hline \hline
\end{tabular}

\subsection{Sequential State Estimation for Nonlinear Systems with Non-Gaussian Noise}

GTEs are nonlinear systems in both the structural state and the performance measurement models, i.e., the state prediction model $\left(\rho_{M}\right)$ is nonlinear, and likewise is the measurement model, which establishes the relation between the internal degradation state $\left(\rho_{M}\right)$ and the measurement indicator $(E R)$. At the same time, the measurements are contaminated with random noise that cannot be accurately parameterized. Such conditions are challenging to identify the internal degradation state of the system, whereas most of state estimation frameworks show poor performance in nonlinear/nonGaussian problems. There is no closed-form algorithm for such systems; however, availability of powerful computational resources facilitates handling the estimation problem numerically. Particle Filter is a numerical process applied on the sequential Monte Carlo method to represent the posterior distribution of the state by a set of weighted sample scenarios (particles), which recursively evolve upon receiving new observations [152]. There are different variants of PF developed to optimize the performance of the state tracking method with reduced computational expense. A recent development on PF is RAPF technique introduced for highly nonlinear/non-Gaussian 
systems [90]. RAPF framework is taken as the platform to develop GTE degradation state estimation with non-uniform time sequences and redundant measurements.

\subsubsection{RAPF Problem Statement}

We consider $x_{k}$ the unobservable internal state of the system and $y_{k}$ the last observation from the observation history $Y_{k} \triangleq\left\{y_{1}, y_{2}, \ldots, y_{k}\right\}$ at time steps: $=1, \ldots, n$. The state $x_{k}$ is a Markov process with a known initial density of $f_{x}\left(x_{0}\right)$, the probability transition density of $f_{x}\left(x_{k} \mid x_{k-1}\right)$ and the marginal distribution of $f_{y}\left(y_{k} \mid x_{k}\right)$. Assuming the density $f_{x}\left(x_{k-1} \mid Y_{k-1}\right)$ is available, then the prior density of the state at the step $\mathrm{k}$ can be estimated as below:

$$
f_{x}\left(x_{k} \mid Y_{k-1}\right)=\int f_{x}\left(x_{k} \mid x_{k-1}\right) f_{x}\left(x_{k-1} \mid Y_{k-1}\right) d x_{k-1}
$$

Bayes' theorem provides the marginal filtering density correspondingly:

$$
f_{x}\left(x_{k} \mid Y_{k}\right)=\frac{f_{y}\left(y_{k} \mid x_{k}\right) f_{x}\left(x_{k} \mid Y_{k-1}\right)}{f_{y}\left(y_{k} \mid Y_{k-1}\right)}
$$

where the normalizing denominator is

$$
f_{y}\left(y_{k} \mid Y_{k-1}\right)=\int f_{y}\left(y_{k} \mid x_{k}\right) f_{x}\left(x_{k} \mid Y_{k-1}\right) d x_{k}
$$

Equations (4.12) through (4.14) constitute the generic Bayesian recursive estimation problem. For the PF technique, at each time step, the posterior density $f_{x}\left(x_{k} \mid Y_{k}\right)$ is numerically represented by $m$ sample scenarios (particles) $x_{k}^{1}, \ldots, x_{k}^{m}$ along with their corresponding weights $\omega_{k}^{1}, \ldots, \omega_{k}^{m}$ :

$$
f_{x}\left(x_{k} \mid Y_{k}\right) \approx \sum_{i=1}^{m} \omega_{k}^{i} \delta\left(x_{k}-x_{k}^{i}\right), \quad\left(\sum_{i=1}^{m} \omega_{k}^{i}=1\right)
$$

where $\delta$ is the Dirac delta function, and the approximation becomes more accurate as $m \rightarrow \infty$ [152]. Upon receiving a new observation $y_{k}$, the particle weights $\omega_{k}^{i}$ can be updated assuming an importance density $g_{x}\left(x_{k} \mid x_{k-1}^{i}, y_{k}\right)$ :

$$
\omega_{k}^{i} \propto \omega_{k-1}^{i} \frac{f_{y}\left(y_{k} \mid x_{k}^{i}\right) f_{x}\left(x_{k}^{i} \mid x_{k-1}^{i}\right)}{g_{x}\left(x_{k}^{i} \mid x_{k-1}^{i}, y_{k}\right)} .
$$

To minimize the weight variance and increase the effective sample size of the particles, 
the optimal importance density should be [153]:

$$
g_{x}\left(x_{k} \mid x_{k-1}^{i}, y_{k}\right)=\frac{f_{y}\left(y_{k} \mid x_{k}, x_{k-1}^{i}\right) f_{x}\left(x_{k} \mid x_{k-1}^{i}\right)}{f_{y}\left(y_{k} \mid x_{k-1}^{i}\right)} \text {. }
$$

Consequently,

$$
\omega_{k}^{i}=\omega_{k-1}^{i} \int f_{y}\left(y_{k} \mid x_{k}\right) f_{x}\left(x_{k} \mid x_{k-1}^{i}\right) d x_{k} .
$$

It is not possible to solve the above integral before knowing the posterior and consequently, it remains as a crucial design step for the PF to choose the importance density. Instead of Equation (4.17), a convenient choice for the importance density is the prior:

$$
g_{x}\left(x_{k} \mid x_{k-1}^{i}, y_{k}\right)=f_{x}\left(x_{k} \mid x_{k-1}^{i}\right)
$$

which is the case in generic PF as well as regularized particle filter (RPF) techniques. It reduces Equation (4.18) to:

$$
\omega_{k}^{i}=\omega_{k-1}^{i} f_{y}\left(y_{k} \mid x_{k}^{i}\right)
$$

In auxiliary particle filter (APF) the importance density is defined the joint density of $f_{x, j}\left(x_{k}, j \mid Y_{k}\right)$, where $\mathrm{j}$ refers to the index of the particle in the previous step $(k-1)$. As a result, we can find the joint density from Bayes' rule:

$$
\begin{aligned}
f_{x, j}\left(x_{k}, j \mid Y_{k}\right) & =f_{y}\left(y_{k} \mid x_{k}\right) g_{x, j}\left(x_{k}, j \mid, Y_{k-1}\right) \\
& =\omega_{k-1}^{j} f_{y}\left(y_{k} \mid x_{k}\right) f_{x}\left(x_{k} \mid x_{k-1}^{j}\right) .
\end{aligned}
$$

As with Equation (4.18), the problem of the unknown posterior holds here. However, the choice of the $j^{\text {th }}$ particle avails an approximation for the posterior, by evolving a representative value $\left(\mu_{k}^{j}\right)$, e.g. mean, mode or a random draw from the prior. Therefore:

$$
f_{x, j}\left(x_{k}, j \mid Y_{k}\right) \approx \omega_{k-1}^{j} f_{y}\left(y_{k} \mid \mu_{k}^{j}\right) f_{x}\left(x_{k} \mid x_{k-1}^{j}\right) \text {. }
$$

Substitution of Equation (4.22) as the importance density in Equation (4.16) yields: 


$$
\omega_{k}^{i} \propto \frac{f_{y}\left(y_{k} \mid x_{k}^{i}\right)}{f_{y}\left(y_{k} \mid \mu_{k}^{j^{i}}\right)},
$$

where, $i$ is the particle index in the current step, while $j$ refers to the index of the particle drawn from the previous step, given the measurements. Apparently, the proposal particles, which have a larger density $f_{j}\left(j \mid y_{k}\right)$ based on their representative values $\left(\mu_{k}^{j}\right)$ are prone to be more drawn, and consequently they constitute a greater importance density.

The RAPF technique attempts to mitigate the shortcomings of the APF in so-called loss of diversity and sensitivity to the outliers in the proposal particles. In conventional APF, the proposal particles $x_{k-1}^{j}$ with a higher probability $\omega_{k-1}^{j} f_{y}\left(y_{k} \mid \mu_{k}^{j}\right)$ will be drawn more frequently than the other particles from the discrete density function. Then the surviving particles evolve to the next step through the prior $f_{x}\left(x_{k} \mid x_{k-1}^{j}\right)$. If the process noise (modeling uncertainty) is small, the selected particles will land in a/some narrow neighborhood(s), which cannot appropriately represent the distribution of the state. In RAPF technique this problem is addressed through regularizing the empirical density $f_{j}\left(j \mid y_{k}\right)$ and redrawing the same population of samples from the continuous distribution. To build a continuous distribution from the proposal particles in the previous step $x_{k-1}^{j}$ with $\omega_{k-1}^{j}$ weights, rescaled Kernel density [154] is employed:

$$
f_{x}\left(x_{k-1} \mid y_{k}\right) \approx \sum_{j=1}^{m} \omega_{k-1}^{j} f_{y}\left(y_{k} \mid \mu_{k}^{j}\right) K_{\mathrm{f}}\left(x_{k-1}-x_{k-1}^{j}\right),
$$

where,

$$
K_{\mathrm{h}}(x)=\mathrm{h}^{-D} K(x / \mathrm{h})
$$

is the rescaled Kernel density with $\mathrm{h}>0$ bandwidth with an optimal value $\mathrm{h}_{\text {opt }}$ for a $D$ dimensional state vector $x$. There are numerous choices for Kernel function $K($.$) and$ Epanechnikov and Gaussian functions are two popular cases.

Equation (4.23) implies that the weight $\omega_{k}^{i}$ can take very large values for the particles whose representative values have small likelihood $f_{y}\left(y_{k} \mid \mu_{k}^{i}\right)$. This nullifies the other particles and impairs the state distribution. On the other hand, the particles with little 
weights play no practical role in the state distribution, while they need the same computational effort as of the other particles. To address the above flaws, an algorithm is employed to control the variance of the weights by rejecting the bad particles, i.e., the particles with the weight: $\omega_{k}^{i}>w$ or $\omega_{k}^{i}<1 / w$, where the weight threshold $w$ is a design parameter [90].

Equations. (4.23) and (4.24) provide the recursive structure of RAPF technique, where the proposal particles of step $k-1$ are drawn from the density provided in Equation (4.24) given the observation in the next step: $k$. The weights are consequently updated by Equation (4.23).

As mentioned earlier, to avoid the particles with very large or very small weights, an acceptance criteria is defined for the weights, and substitutive proposals have to be drawn until they comply. In practice, it is very hard to successfully draw proposal $\left(x_{k}^{i}\right)$ with acceptable weight, given the same representative value for the particles $\left(\mu_{k}^{i}\right)$, because the corresponding likelihood $f_{y}\left(y_{k} \mid \mu_{k}^{i}\right)$ is small anyway. Consequently, a practical way to reject inappropriate proposals is to bound the likelihood of the representative values, as outlined in Table 4.2.

The RAPF technique outperforms RPF and APF in some nonlinear/non-Gaussian benchmark systems [90] and offers a competent potential for internal state estimation for a nonlinear system with noisy measurements, such as a GTE.

Table 4.2. RAPF technique with rejection of low likelihood corresponding particles

$$
\left(x_{k}, \omega_{k}\right)=\operatorname{RAPF}\left[\left(x_{k-1}, \omega_{k-1}\right), y_{k}\right]
$$

- Estimate: $\quad \mu_{k}=E\left(x_{k} \mid x_{k-1}\right)$

- Find: $\quad \omega_{\mu_{k}} \propto \omega_{k-1} f_{y}\left(y_{k} \mid \mu_{k}\right)$

- Resample: $\left(\mu_{k}, 1 / m\right)=\operatorname{Resample}\left(\mu_{k}, \omega_{\mu_{k}}\right)$

- Reject the representative particle with low likelihood: $f_{y}\left(y_{k} \mid \mu_{k}\right) \ll 1$ and substitute from "Resample".

- Draw: $\quad x_{k} \sim f_{x}\left(x_{k} \mid \mu_{k}\right)$

- Find: $\quad \omega_{k} \propto f_{y}\left(y_{k} \mid x_{k}\right) . / f_{y}\left(y_{k} \mid \mu_{k}\right)$

- Reject the proposal particle if corresponding $\omega_{k}>w$ or $\omega_{k}<1 / w$ and return to "Draw". 


\subsubsection{RAPF in Non-Uniform Time Steps and Redundant Measurements}

The majority of sequential state estimation techniques, including PF variants work with uniform time series, i.e., the data collected in equal time segments. In some occasions, the data is not logged regularly due to any reason. In this study, the measurement data pertain to the limited hours after the compressor washes, which did not happen on a regular basis, as seen in table 4.1. The state model should consequently consider the time length of each segment. Assuming $x_{k-1}$ the state variable at the time $t_{k-1}$, the state at $t_{k}$ and the consequent observation can be termed as below:

$$
\begin{aligned}
& x_{k}=F\left(x_{k-1}\right)+\tau_{k}, \\
& y_{k}=G\left(x_{k}\right)+v_{k},
\end{aligned}
$$

where $F($.$) and G($.$) are the state and the measurement models respectively, and \tau_{k}$ and $v_{k}$ are the process noise (modeling uncertainty) and the measurement noise respectively. For a time model as in Equation (4.10), assuming $\Delta t_{k}=t_{k}-t_{k-1}$ the variable time length in the $k^{\text {th }}$ segment:

$$
\rho_{M}\left(t_{k}\right)=e^{b \Delta t_{k}} \rho_{M}\left(t_{k-1}\right)+a\left(1-e^{b \Delta t_{k}}\right) .
$$

The state model, using the actual internal state $\rho_{R}$ instead of $\rho_{M}$ is therefore

$$
\rho\left(t_{k}\right)=e^{b \Delta t_{k}} \rho\left(t_{k-1}\right)+a\left(1-e^{b \Delta t_{k}}\right)+\tau_{k} .
$$

Recalling $\varepsilon$ from Equation (4.11) and using Equations (4.27) and (4.28), the process noise is yielded:

$$
\tau_{k}=\varepsilon\left(t_{k}\right)-e^{b \Delta t_{k}} \varepsilon\left(t_{k-1}\right),
$$

which can be numerically calculated as the representative values, using table 4.1. At each time step, the random noise of the process is consequently a draw from the following distribution:

$$
\begin{aligned}
f_{\tau}(\tau) & =\sum_{k=1}^{n} \delta\left(\tau-\tau_{k}\right), \\
& \approx \sum_{k=1}^{n} K_{\mathrm{h}}\left(\tau-\tau_{k}\right) .
\end{aligned}
$$

In typical sequential state estimation techniques, at each time step, a single 
measurement is performed, which contains a noise with a definite statistical characteristics. Assume a system with unknown behavior of the sensors, where the sequential observation is performed on an indicator synthesized from multiple noisy signals in a complex mathematical structure. It is impractical to extract the noise characteristics of such a system analytically. The mentioned conditions hold for the GTE in the study and need to be addressed.

Assume $G\left(x_{k}\right)$ a deterministic monotonic function for measurement. From Equation (4.26), the measurement density is

$$
\begin{aligned}
f_{y}\left(y_{k} \mid x_{k}\right) & =f_{y}\left(G\left(x_{k}\right)+v_{k} \mid x_{k}\right) \\
& =f_{v}\left(v_{k} \mid x_{k}\right) .
\end{aligned}
$$

This implies that, if enough measurement samples in a given state are available, measurement noise can be empirically characterized by Equation (4.31). On this basis, at each time step $t_{k}$, we take $s$ consecutive samples, i.e., $E R\left(t_{k}\right)_{l},(l=1, \ldots, s)$, immediately after the compressor wash. It is reasonable to assume that during the short time of sampling, the degradation state of the system does not change and the variation of the samples roots only in the measurement noise. The likelihood density of the measurement is consequently

$$
f_{E R}\left(E R\left(t_{k}\right) \mid \rho\left(t_{k}\right)\right) \approx \sum_{l=1}^{s} K_{\mathrm{f}}\left(E R\left(\rho\left(t_{k}\right)\right)-E R\left(t_{k}\right)_{l}\right)
$$

Using equations (4.28), (4.30) and (4.32) in the algorithm presented in Table 4.2, we employ the RAPF framework in non-uniform time sequences and redundant measurements of the GTE. Given the latest set of consecutive samples for $\operatorname{ER}\left(t_{k}\right)_{1: s}$ becomes available from the GTE measurements at $t_{k} \approx$ present time, the developed framework will update the state estimation for real-time monitoring.

\subsection{Performance Evaluation}

In this section, the developed framework for state estimation is firstly examined by simulations based on a modified benchmark model and then applied on the actual GTE measurements. To evaluate its performance, the results are consequently compared with RPF technique in each case. 


\subsubsection{Benchmark Model with Non-Uniform Steps and Redundant Measurements}

The following benchmark model is widely used for performance evaluation of sequential filters [155]:

$$
\begin{aligned}
& x_{k}=\frac{1}{2} x_{k-1}+\frac{25 x_{k-1}}{1+x_{k-1}^{2}}+8 \cos [1.2(k-1)]+\tau_{k} \\
& y_{k}=\frac{1}{20} x_{k}^{2}+v_{k}
\end{aligned}
$$

where $\tau_{k}$ and $v_{k}$ are Gaussian white noise and the length of the time steps has no influence on the state model. We introduce the extend form of the benchmark model for non-uniform time step length as the following:

$$
x_{k}=\frac{1}{2} x_{k-1} \Delta t_{k}+\frac{25 x_{k-1} \Delta t_{k}}{1+\left(x_{k-1} \Delta t_{k}\right)^{2}}+8 \Delta t_{k} \cos [1.2(k-1)]+\tau_{k} \text {, }
$$

where $\Delta t_{k}=t_{k}-t_{k-1}$ is the random time length between steps $k-1$ and $\mathrm{k}$ with an arbitrary distribution, likewise is the distribution of the process noise $\tau_{k}$. Assume the same measurement model of Equation (4.34), where there is no parameterized distribution for the measurement noise $v_{k}$, and instead, there is a set of $s$ redundant measurements: $\left\{y_{k}^{l}\right\}_{l=1}^{s}$ at each step. To run the model for a test case, assume $\Delta t_{k} \sim \mathcal{U}(0,2)$ and $\tau_{k} \sim \mathcal{N}(0,5)$, which simulates a very noisy process. For the measurements, we generate 100 samples from a randomly weighted average of three different distributions, i.e., Normal, Gamma and Rayleigh, and the random weights are drawn from a uniform distribution. The parameters of the distributions are set such that: $E\left(y_{k}^{l}\right)=\frac{1}{20} x_{k}^{2}$ and $\operatorname{Var}\left(y_{k}^{l}\right)=10$ at each step $k$, which corresponds to a highly noised measurement. We generate a sample series of the state at 50 non-uniform time steps. To evaluate the performance of RAPF and RPF techniques, we run each of the state estimation techniques with 200 particles for 20 rounds. The results are shown in Figure 4.5(a) and (b) respectively. As it is seen in the plots, the RAPF technique shows a better repeatability compared to RPF. Quantitatively, the mean square of the standard deviation of the estimated states is 0.0747 for RAPF, compared to 0.0995 for RPF. In terms of the 
absolute error, we compare the mean square errors of the results. RAPF shows an absolute error of 1.8940 , while this error is 2.1325 for RPF. The overall performance of RAPF is superior, however, RPF may provide comparable estimations in some occasions, e.g. $t_{8}=7.96$, and $t_{24}=24.17$. It should be noted that the state is a random variable as defined in Equation (4.35), and it may accidently be located in the vicinity of RPF prediction. As a result, a good performance can be observed occasionally for RPF at such instances.

(a)

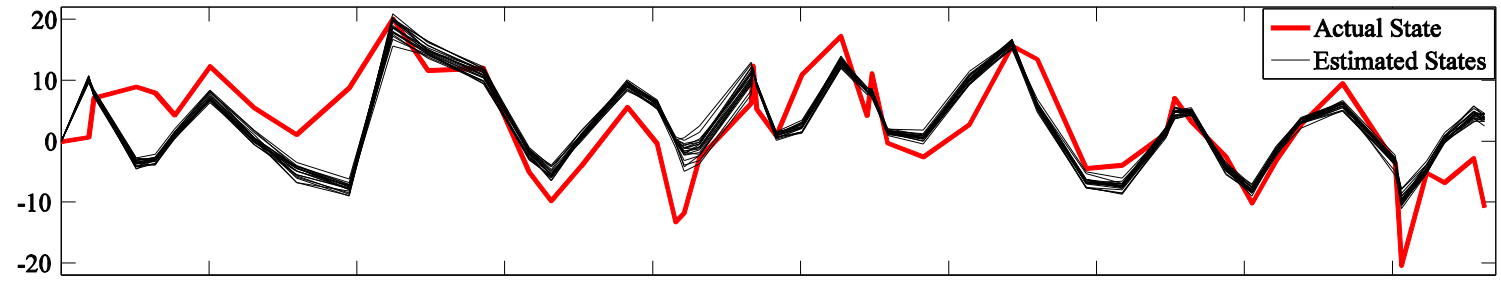

(b)

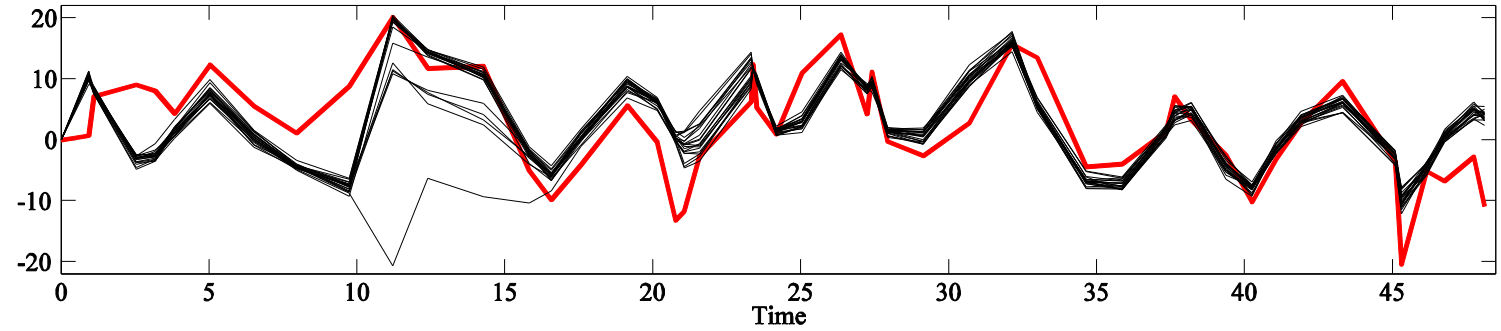

Figure 4.5. Performance of the state estimation techniques: (a) RAPF technique, (b) RPF technique.

\subsubsection{Performance Evaluation on Gas Turbine Data}

The degradation state model of the gas turbine is presented in Equation (4.28) with parameters $\mathrm{a}$ and $\mathrm{b}$, mentioned in section 4.2 .2 , and the process noise is characterized by Equation (4.30). Figure 4.6 shows the degradation model and its uncertainty, which is independent from the sequential measurements.

For the measurements, there are sets of redundant observation samples after each wash of the compressor (see Figure 4.4(a)). To assign the sample size, one should consider enough sample population to properly represent the likelihood. At the same time, the duration of sampling should be short enough to be not affected by the performance deterioration. For this reason, different sample sizes were examined and a size of 60 samples which is equivalent to 5 day data logging from the GTE operating system was 
deemed to be optimal. Figure 4.7 shows the likelihood density of the measurements depicted with 200 sample particles in selected sequences.

Having the model and the measurement distribution, we apply RAPF to estimate the state of degradation. Figure 4.8 shows the state particles and their corresponding weights in the same selected sequences of the previous figure. We observe that particles associated with higher measurement likelihoods hold larger weights, while the density of the particles in such regions is acceptably high. This means, the particles are such distributed as to effectively represent the probability density.

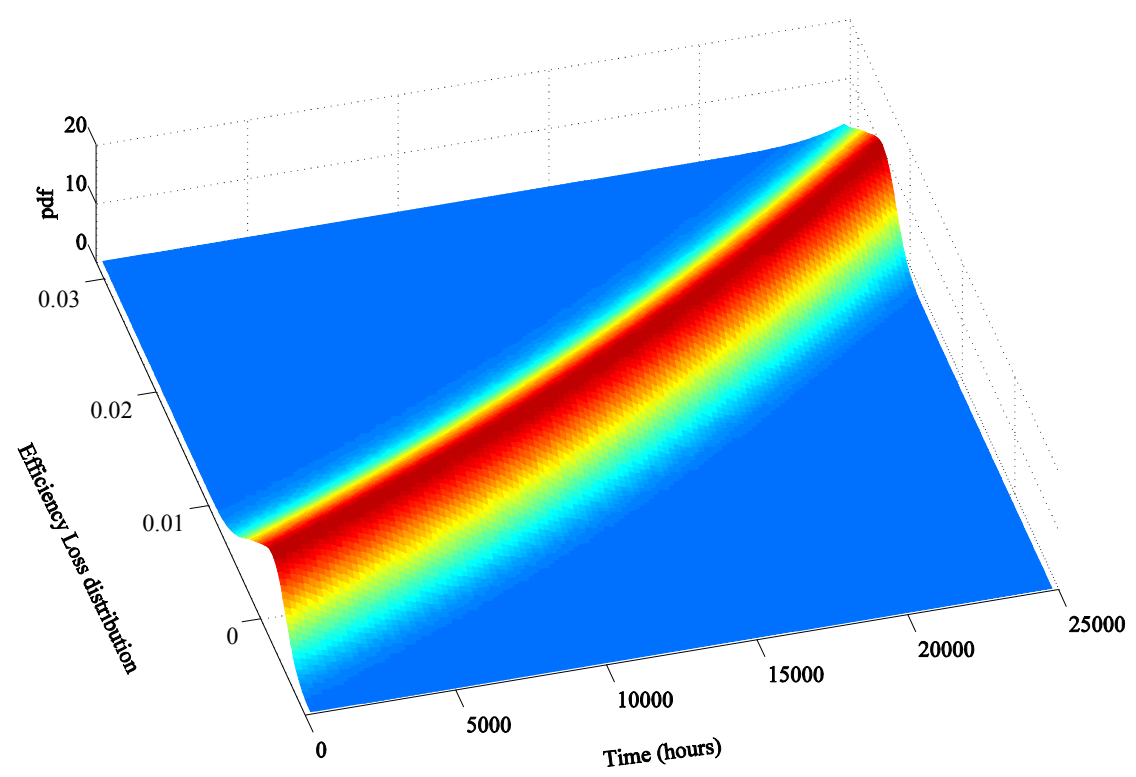

Figure 4.6. Degradation model and modeling uncertainty pdf.

Eventually, the trend of the estimated ratio of the isentropic efficiency loss $(\rho)$, using 200 particles is provided in Figure 4.9(a). A quarter of the trajectories are shown in green and the trend of the expected $\rho$ value is depicted in blue. The result shows a smooth growth trend, except for the steps 8 and 14, where there is a slight reduction in $\rho$. Due to the non-recoverable degradation mechanisms in the turbine section, the retrieval of the isentropic efficiency ratio is not expected, unless a maintenance service is provided to the GTE in addition to the regular compressor wash. This can be further investigated, if the service logs of the GTE are available. The result shows that the isentropic efficiency of 
the turbine section decreases about 3\% during the entire time between overhauls (TBO) cycle, which is in the range of the losses reported in the literature $[142,156]$.

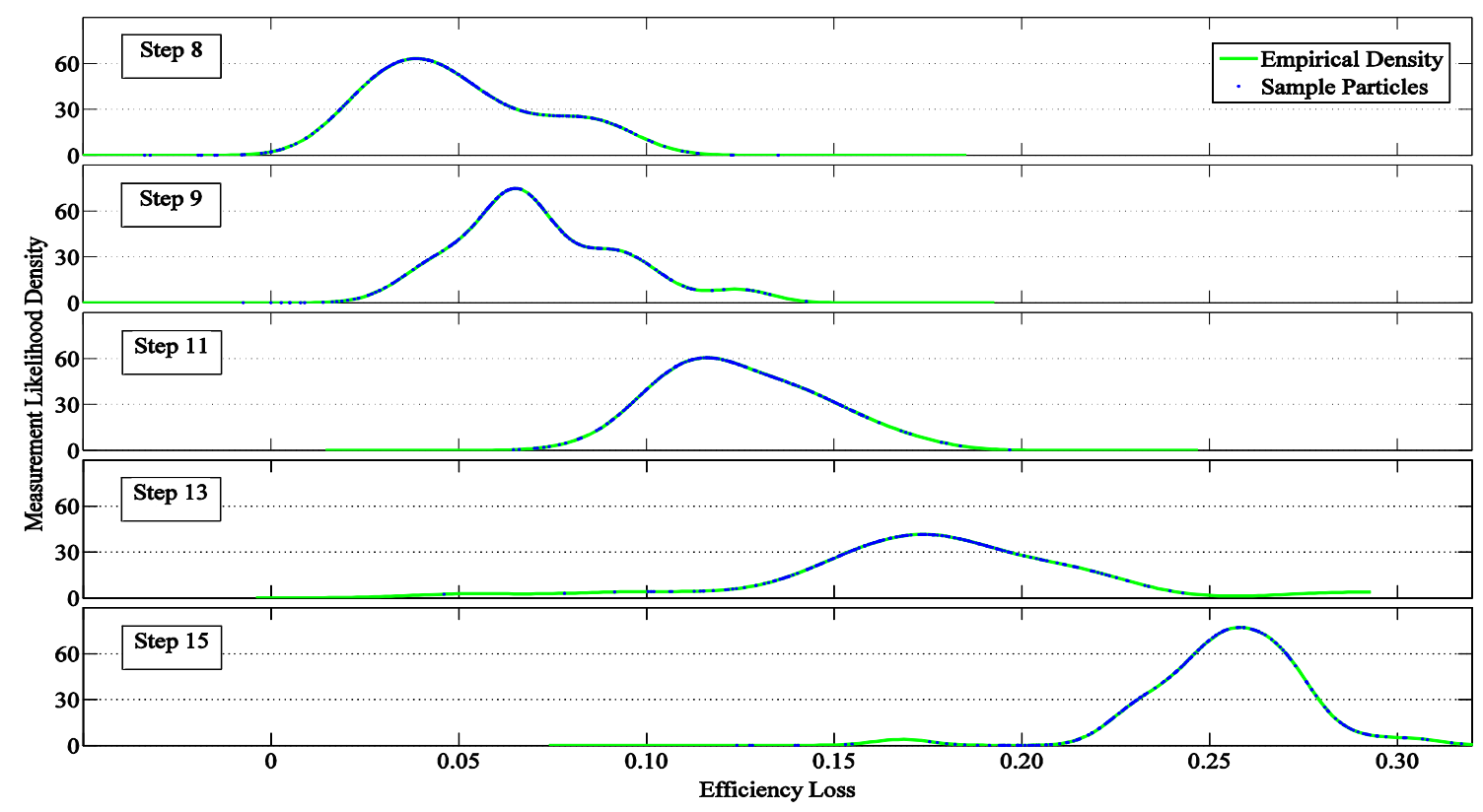

Figure 4.7. Measurement likelihood empirical density in different steps.

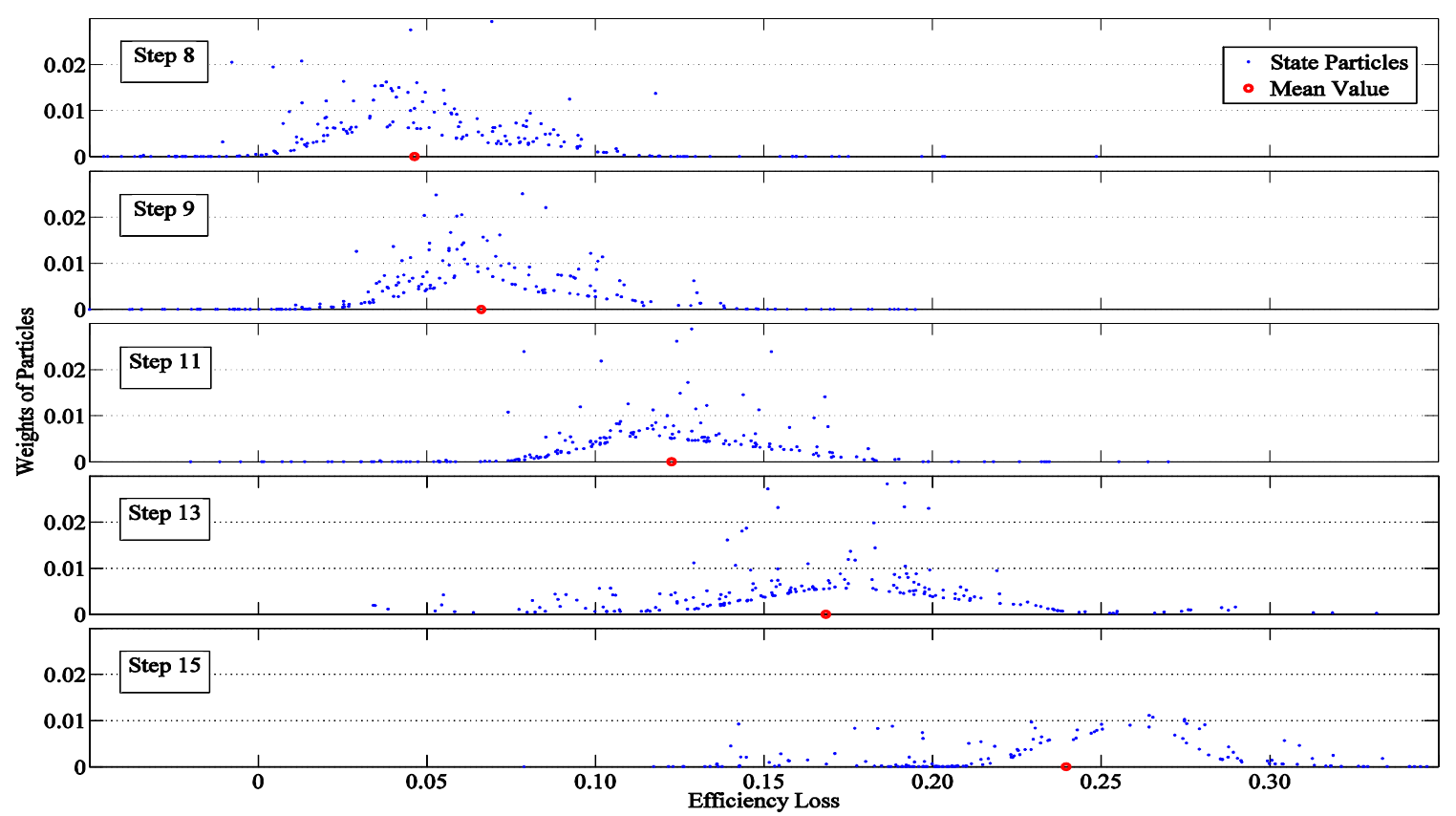

Figure 4.8. Distribution of the state particles and corresponding weights. 
As a comparative result, RPF technique is applied on the same data and the result is plotted in red-dashed line. The RAPF technique shows a rather smoother trend. In addition, the technique shows a better repeatability through 20 times run on the same data. Figure 4.9(b) shows the standard deviation of the repetitive results at each time step, where RAPF shows a better repeatability in almost all the steps. As the overall result, the expected standard deviation of the state estimations is 0.0295 for RAPF, compared to 0.0370 for RPF.

(a)
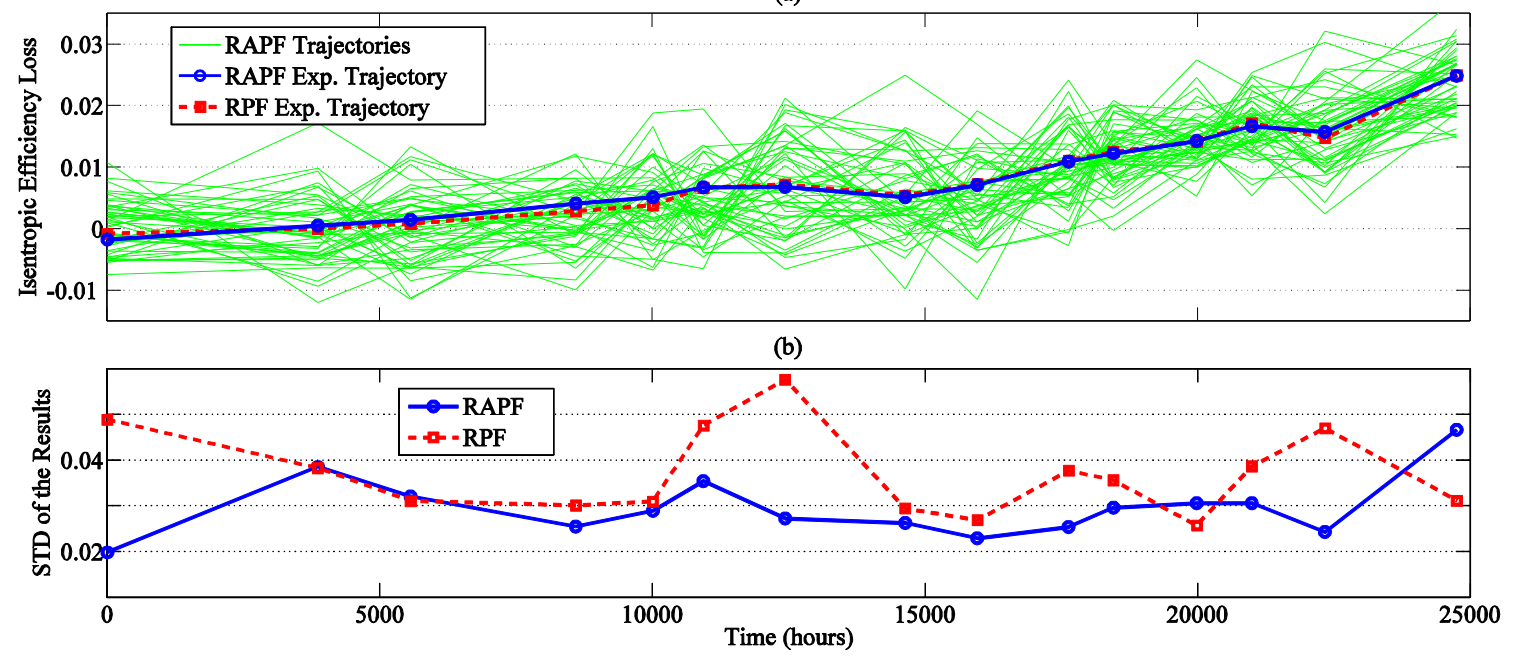

Figure 4.9. Comparative results :(a) RAPF trajectories with 200 particles (green) and the expected trajectory (blue), compared with the expected RPF trajectory (red), and (b) Comparison of repeatability: standard deviation for 20 runs.

\subsection{Summary}

In this chapter, a physics-based performance indicator was introduced based on the thermodynamic model of a single shaft simple-cycle GTE. The developed indicator was able to represent the performance deterioration for both short-term and long-term degradations arising from the compressor fouling and the turbine structural degradation respectively. Ratio of the isentropic efficiency loss in the turbine section was defined as the internal degradation state for the turbine and its effect on the introduced performance indicator was formulated by the measurement model. To estimate the most probable 
internal state in non-uniform time steps, where the measurement noise has unknown characteristics, a RAPF framework was developed for sequential state estimation. The effectiveness of the developed framework was examined on two cases: a benchmark model with known internal states, and on the operating data of a GTE during three years of operation. In both cases, the developed framework showed a superior performance in accuracy and repeatability, compared to the other framework based on RPF technique. For the GTE internal degradation state estimation, the technique could clearly track the ratio of the isentropic efficiency loss during the GTE operating time and showed near 3\% loss after a three-year operating period.

The developed framework extends the application of RAPF technique to the nonuniform time steps and it manages the problems with an unknown measurement noise characteristic. 


\section{Chapter 5: Effects of Humidity Condensation on the Trend of Gas Turbine Performance Deterioration}

\subsection{Overview}

An effective predictive maintenance plan entails a comprehensive knowledge about the trend of performance deterioration, especially the short-term regime, which is required for planning the sequential compressor washing. Various factors such as air pollution, intake air filter condition, and humidity of air are effective in the trend of shortterm performance deterioration [141]. Stalder reported the humidity as a contributing factor to the deterioration rate, however, an inverse effect is sometimes observed, where the performance improves due to the humidity [157]. Despite a few studies mentioned above, there is still a vast unexplored area regarding the operating factors and the form of their effects on the GTE performance deterioration. Consequently, based on the available knowledge in the literature, the expected performance level of the GTE cannot be accurately predicted from the history of the operating and ambient conditions.

In this chapter, the dependency of the trend of the short-term performance deterioration on the operating and the ambient conditions of a GTE is investigated. The proposed study incorporates the following aspects: 1) the utilized performance indicators unify the health level of the GTE in a single value with an insightful physical meaning; and 2) the combined effects of the ambient and the operating conditions are studied, and the effect of the humidity condensation rate in the trend of performance deterioration is quantitatively investigated.

\subsection{Physics-based modeling}

A detailed thermodynamic model for the single shaft GTEs with humid air was developed in Chapter 3, which uses the operating parameters, i.e., intake air total temperature $\left(T_{C i}\right)$, total pressure $\left(P_{C i}\right)$ and specific humidity $\left(S H_{C i}\right)$, shaft speed $(N)$, and fuel to air mass ratio $(f)$. By calibrating the model with the GTE design parameters, the model simulates the operating conditions and predicts the unknown gas path parameters $(G P P)$ : 


$$
\left[G P P_{M}\right]=\mathrm{F}_{G T E_{M}}\left(T_{C i}, P_{C i}, S H_{C i}, N, f\right) .
$$

In many gas turbines, the specific fuel consumption $f$ is not measured by the operating system. To adjust the model for such applications, $f$ can be cancelled from the equations in two ways: 1) by setting $E G T_{M}$ equal to the measured $E G T$ and find the corresponding $f$, which reduces Equation (5.1) to:

$$
P W_{G T E_{M}}=P W_{G T E_{M}}\left(T_{C i}, P_{C i}, S H_{C i}, N, E G T\right),
$$

and, 2) by setting $P W_{G T E_{M}}$ equal to the measured $P W$ and use the corresponding $f$, which results:

$$
E G T_{M}=E G T_{M}\left(T_{C i}, P_{C i}, S H_{C i}, N, P W\right) .
$$

\subsubsection{Performance Indicators}

Two unified physics-based performance indicators were developed in Chapters 3 and 4, which quantify the health level of the GTE using the mentioned operating data.

Excess Heat Ratio: Using Equation (5.3), excess heat ratio $(E H)$ was defined in Chapter 3 as the normalized value of the extra enthalpy discarded from a GTE exhaust, compared to the ideal condition:

$$
\begin{aligned}
E H & =\left(\dot{H}_{T o}-\dot{H}_{T o_{M}}\right) / P W_{G T E_{D}} \\
& =W_{T} c_{p_{M i x}}\left(E G T-E G T_{M}\right) / P W_{G T E_{D}} .
\end{aligned}
$$

where $W_{T}$ is the exhaust gas mass flow and $c_{p_{M i x}}$ is the average specific heat capacity of the exhaust gas in the range of the measured and the modeled temperature. This indicator quantifies the ratio of the extra thermal energy, a GTE is losing, due to its performance deterioration.

Efficiency Ratio: In Chapter 4, efficiency ratio $(E R)$ was introduced as another performance indicator and defined as the ratio of the actual thermal efficiency $\left(\eta_{G T E_{A}}\right)$ and the thermal efficiency predicted by the model in the same operating conditions $\left(\eta_{G T E_{M}}\right)$ : 


$$
E R=\eta_{G T E_{A}} / \eta_{G T E_{M}} .
$$

Thermal efficiency of the GTE is predicted by the model, given the lower calorific value of the fuel $(L C V)$ :

$$
\eta_{G T E_{M}}=P W_{G T E_{M}} /\left(W_{F_{M}} L C V\right) .
$$

and it was shown that actual thermal efficiency of the GTE is:

$$
\eta_{G T E_{A}}=P W /\left(\left(W_{F_{M}} L C V\right)+P W-P W_{G T E_{M}}\right) .
$$

This indicator provides a scale, showing how efficient is an actual GTE, compared to its ideal health condition.

Figure 5.1 shows the results of applying $E H$ and $E R$ performance indicators on the operating data of the gas turbine in the study. Accordingly, both indicators show the GTE performance steeply deteriorates in short-terms and then abruptly resumes close to the initial level. This pattern happens due to the fouling and consequent cold wash of the engine core. In the longer-term however, the gradual increasing trend of the indicators show the deterioration of the performance, which has not been recovered by washing services and can be accounted to the irreversible structural degradation of the engine parts.

(a)

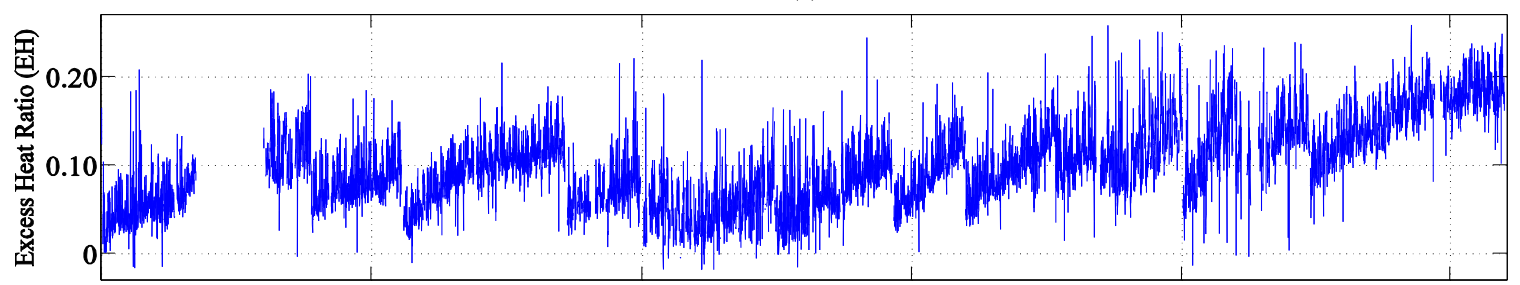

(b)

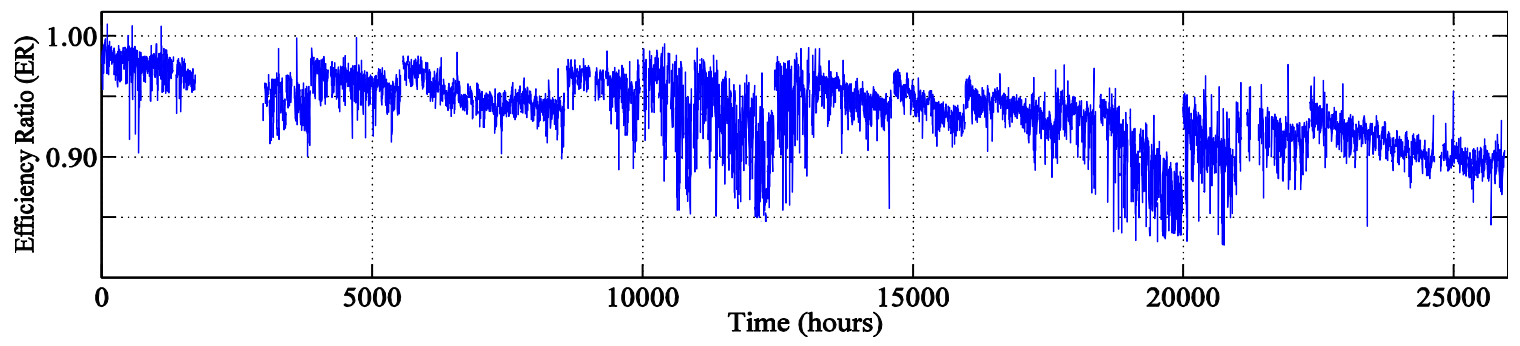

Figure 5.1. Performance deterioration during the operating life. (a) $E H$ performance indicator, and (b) $E R$ performance indicator. 


\subsubsection{Humidity Condensation}

To improve the performance of the gas turbines during the warm days, water is often sprayed into the intake air stream. The added water, plus the initial humidity content of air can lead the condition towards the dew point. At the same time, when air accelerates in the intake duct, its static pressure and temperature drop, and consequently, its capacity to hold the humidity in gaseous form decreases. If the total humidity content of the air exceeds the mentioned capacity limit, the extra humidity condenses as mist and enters the compressor. The mist can reach the first stages of the compressor and affect the circumstances, which contributes to the congestion of the foulants on the blades and vanes. To study this phenomenon, the rate of humidity condensation is to be determined.

Assuming that air accelerates from the stationary condition to the velocity $V_{C i}$ at the inlet of the compressor, the change of the specific enthalpy using the first law of thermodynamics is

$$
h_{a m}-h_{C i}=V_{C i}^{2} / 2 \text {. }
$$

In small ranges of temperature variation, the specific enthalpy is an affine function of the temperature: $h_{a m}-h_{C i}=c_{p}\left(T_{a m}-T_{s_{C i}}\right)$. Where $T_{s_{C i}}$ is the static air temperature at the compressor inlet. The average velocity of air at the compressor inlet is $V_{C i}=$ $v_{C i} W_{C i} / A_{C i}$, where $W_{C i}$ is the inlet air mass flow, passing through a cross sectional area of $A_{C i}$, and $v_{C i}$ is the specific volume of the inlet air, which is a function of temperature for the isentropic process

$$
v_{C i}=v_{a m}\left(T_{a m} / T_{s_{C i}}\right)^{1 /(1-\gamma)} .
$$

The specific volume for a perfect gas with the constant $R$ is: $v_{a m}=R T_{a m} / P_{a m}$, and as a result, the implicit equation for static temperature of the compressor inlet flow, and corresponding static pressure will be found as

$$
\begin{aligned}
& T_{s_{C i}}=T_{a m}-\left(W_{C i} R T_{a m}^{\gamma /(\gamma-1)} T_{S_{C i}}^{1 /(1-\gamma)} / A_{C i} P_{a m}\right)^{2} /\left(\begin{array}{ll}
2 & c_{p}
\end{array}\right), \\
& P_{s_{C i}}=P_{a m}\left(T_{s_{C i}} / T_{a m}\right)^{\gamma /(\gamma-1)} .
\end{aligned}
$$

The saturation vapor pressure in the air $P_{w}^{*}[\mathrm{~Pa}]$ is suggested as a function of the 
temperature $T_{S}\left[{ }^{\circ} \mathrm{C}\right]$ by Buck [149].

$$
P_{w}^{*}=611.21 \exp \left(\left(18.678-T_{s} / 234.5\right)\left(T_{s} /\left(257.14+T_{s}\right)\right)\right) .
$$

The maximum specific humidity at such saturation pressure is [148]

$$
S H_{\max }=0.622 P_{w}^{*} /\left(P_{S_{C i}}-P_{w}^{*}\right) .
$$

If the actual humidity content is more than the maximum limit, the extra humidity condenses as mist with a condensation rate of

$$
W_{c o n}=W_{C i}\left(S H_{C i}-S H_{\max }\right) .
$$

Equations (5.10) through (5.14) provide the condensation rate of the inlet air humidity as a function of the ambient temperature and pressure, specific humidity of the inlet flow, the mass flow and the cross sectional area of the inlet duct. For the gas turbine in the study, the cross sectional area of the compressor inlet is $A_{C i}=0.11 \mathrm{~m}^{2}$. Using the ambient temperature and pressure from the logged data, and using the model results for the mass flow and its specific humidity in Chapter 3 [158], the condensation rate is calculated for the operating period of the gas turbine as shown in Figure 5.2(b). Figure 5.2(a) shows the total humidity in the inlet air after the cooler, and Figure 5.2(c) shows both total and condensed humidity in a selected period for comparison. The variation in the total humidity content is mainly due to the variation of the temperature. As it is seen, the humidity content rises in the summers, and as a result, extra humidity condenses with a larger rate in the inlet duct, compared to the cold seasons. Condensation rate varies from 0 to over $0.2 \mathrm{~kg} / \mathrm{s}$ depending on the condition. 
(a)

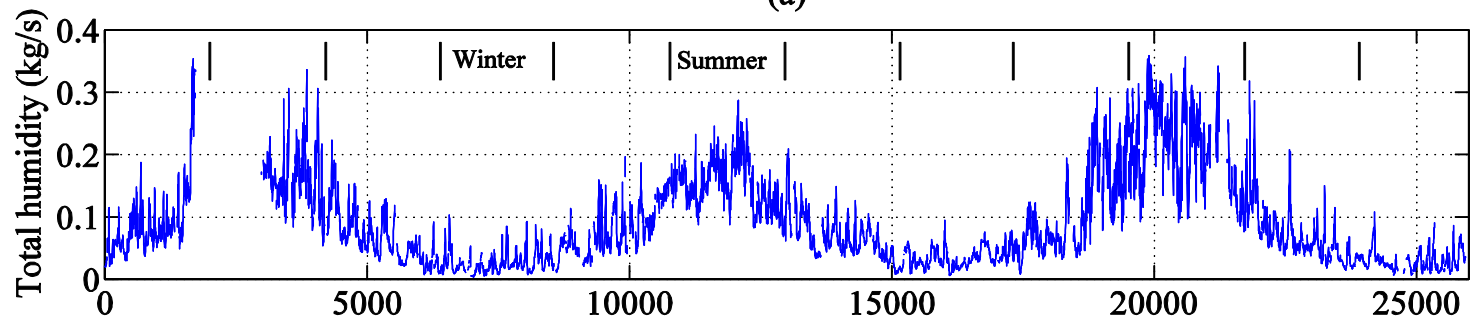

(b)

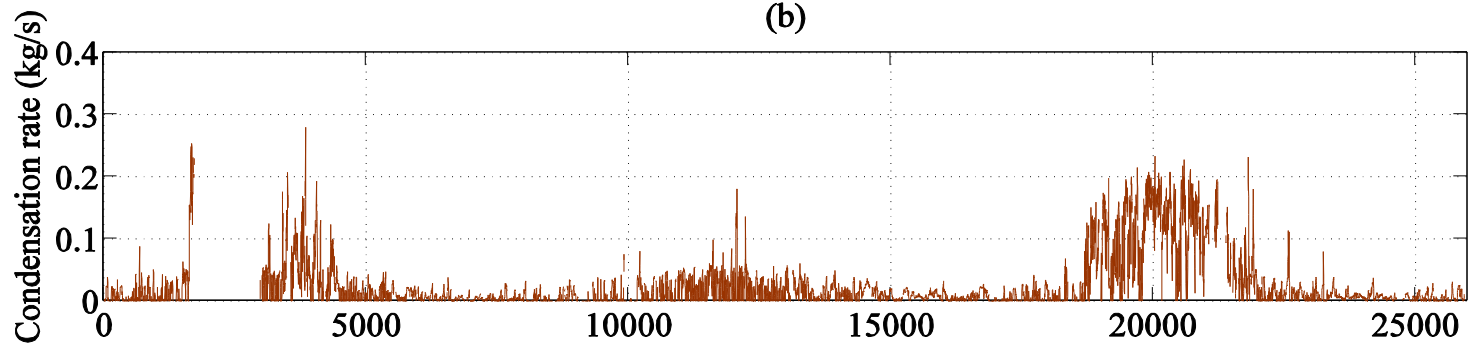

(c)

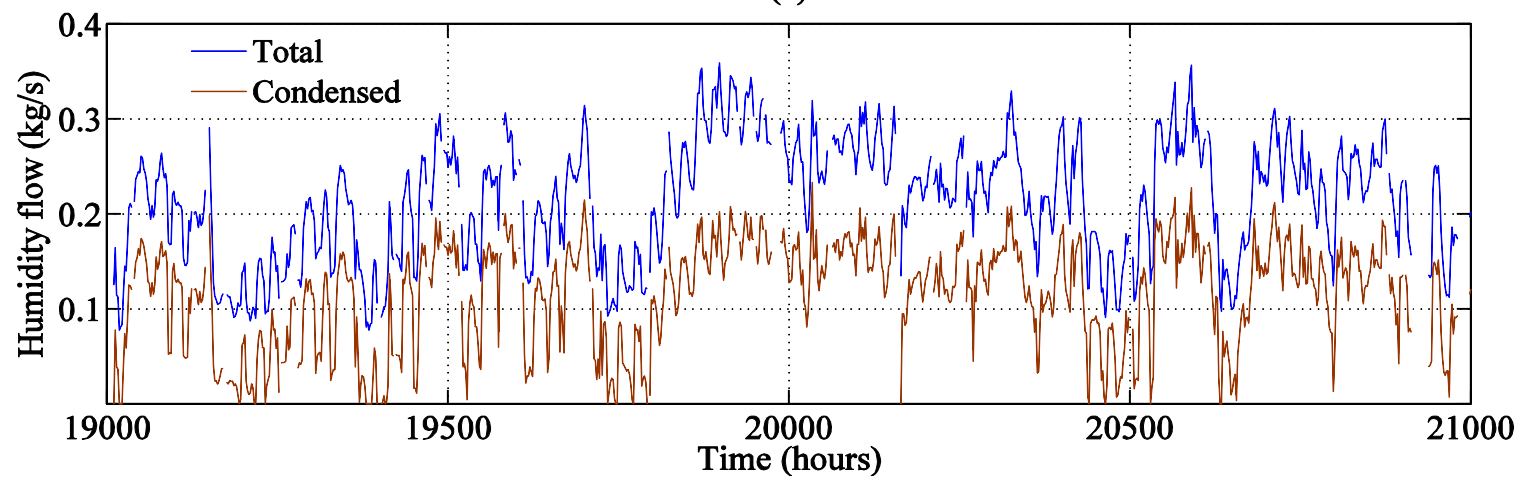

Figure 5.2. Humidity in inlet air: (a) total humidity, (b) condensed humidity, and (c) comparison in elected time window.

\subsection{Data-Model Fusion Framework}

The performance indicator signals are noisy as shown in Fig 5.1. An effective way for noise filtration is to utilize a data-model fusion technique, which estimates the state (here the signal) with a higher accuracy than the individual measurement or the model. Kalman Filter (KF) is a classic version of data-model fusion technique that provides a closed form solution for the state estimation. However, the inability to handle nonlinear and/or nonGaussian systems limits its applications for the cases such as nonlinear fouling phenomena in this work [155]. Hence, a particle filter (PF) framework is being proposed in this work, which is capable of dealing with nonlinearity of the fouling as well as stochastic noise signatures [152]. 


\subsubsection{Particle Filter}

PF is the numerical simulation of the sequential Monte Carlo method for state estimation where the posterior distribution of the state variables are represented by a number of weighted particles. The estimated state distribution is evolved recursively as the weight of the particles are updated when a new observation becomes available [90]. Assume a Markov process where $x_{k}$ is the internal state with a known initial density of $f_{x}\left(x_{0}\right)$, and $y_{k}$ is the last observation from the observation history $Y_{k} \triangleq\left\{y_{1}, y_{2}, \ldots, y_{k}\right\}$ at time step $k$. Given the probability density of $f_{x}\left(x_{k} \mid x_{k-1}\right)$ for transition process and the posterior density $f_{x}\left(x_{k-1} \mid Y_{k-1}\right)$ at the previous time step, the prior density of the state will be

$$
f_{x}\left(x_{k} \mid Y_{k-1}\right)=\int f_{x}\left(x_{k} \mid x_{k-1}\right) f_{x}\left(x_{k-1} \mid Y_{k-1}\right) d x_{k-1}
$$

The posterior density at the current step is then found by Bayes' theorem given the marginal distribution density of the measurement $f_{y}\left(y_{k} \mid x_{k}\right)$ :

$$
f_{x}\left(x_{k} \mid Y_{k}\right)=f_{y}\left(y_{k} \mid x_{k}\right) f_{x}\left(x_{k} \mid Y_{k-1}\right) / f_{y}\left(y_{k} \mid Y_{k-1}\right)
$$

where the normalization factor in the denominator is

$$
f_{y}\left(y_{k} \mid Y_{k-1}\right)=\int f_{y}\left(y_{k} \mid x_{k}\right) f_{x}\left(x_{k} \mid Y_{k-1}\right) d x_{k}
$$

In $\mathrm{PF}$, the state distribution is represented by m sample particles $x_{k}^{1}, \ldots, x_{k}^{m}$ and their corresponding weights $\omega_{k}^{1}, \ldots, \omega_{k}^{m}$, therefore the posterior density will be

$$
f_{x}\left(x_{k} \mid Y_{k}\right) \approx \sum_{i=1}^{m} \omega_{k}^{i} \delta\left(x_{k}-x_{k}^{i}\right)
$$

where $\delta$ is Dirac delta function. By choosing the importance density of the particles equal to their probability density, the weights of the particles are updated

$$
\omega_{k}^{i \prime}=\omega_{k-1}^{i} f_{y}\left(y_{k} \mid x_{k}^{i}\right)
$$

which should be normalized to meet $\sum_{i=1}^{m} \omega_{k}^{i}=1$

$$
\omega_{k}^{i}=\omega_{k}^{i \prime} / \sum_{i=1}^{m} \omega_{k}^{i \prime}
$$

A common problem after some iterations is the degeneracy phenomenon, where a few particles hold the major weights and the others practically remain with no weights. To 
avoid this phenomenon a re-sampling step can be added to the process right after updating the weights. To perform re-sampling in conventional PF, the posterior density is constructed by Equations (5.18) and (5.20) and redraw the particles from the discrete posterior density. In order to diversify the particles and not repeat drawing a single particle for several times, the posterior density can be reconstructed in a continuous form. This technique is called regularized particle filter (RPF) where the continuous posterior density is defined by a rescaled Kernel density rather than Dirac delta function

$$
f_{x}\left(x_{k} \mid Y_{k}\right) \approx \sum_{i=1}^{m} \omega_{k}^{i} K_{\mathrm{f}}\left(x_{k}-x_{k}^{i}\right),
$$

where,

$$
K_{\mathrm{h}}(x)=\mathrm{h}^{-D} K(x / \mathrm{h})
$$

is the rescaled Kernel density with bandwidth $\mathrm{h}>0$ and the optimal value $\mathrm{h}_{\text {opt }}$ for a $D$ dimensional state vector $x$.

In a practical case, assume the state transition is modeled by a deterministic function $F_{k}($.$) with a random process noise of \tau_{k}$, with a known distribution

$$
x_{k}=F_{k}\left(x_{k-1}\right)+\tau_{k},
$$

and the observation is also modeled by a deterministic model $G_{k}($.$) . The observation$ always contains a noise component $v_{k}$ with a presumed distribution:

$$
v_{k}=y_{k}-G_{k}\left(x_{k}\right) .
$$

For an individual particle $x_{k-1}^{i}$, Equation (5.23) predicts the state by drawing a random value for the process noise $\tau_{k}^{i}$ from its distribution. To update the weight through Equation (5.19), Equation (5.21) is employed to find the likelihood

$$
\begin{aligned}
f_{y}\left(y_{k} \mid x_{k}^{i}\right) & =f_{y}\left(G_{k}\left(x_{k}^{i}\right)+v_{k}^{i} \mid x_{k}^{i}\right) \\
& =f_{v}\left(v_{k}^{i} \mid x_{k}^{i}\right) .
\end{aligned}
$$

Assuming the measurement noise is independent from the state

$$
\omega_{k}^{i \prime}=\omega_{k-1}^{i} f_{v}\left(v_{k}^{i}\right) .
$$

Table 5.1 outlines the recursive RPF process. 
Table 5.1. RPF technique process flow

$$
\left(x_{k}^{i}, \omega_{k}^{i}\right)=\operatorname{RPF}\left[\left(x_{k-1}^{i}, \omega_{k-1}^{i}\right), y_{k}\right], \quad i=\{1, \ldots, m\}
$$

- Draw the process noises: $\quad \tau_{k}^{i} \sim f_{\tau}\left(\tau_{k}\right)$

- Predict the next step particles: $\quad x_{k}^{i}=F_{k}\left(x_{k-1}^{i}\right)+\tau_{k}^{i}$

- Find the measurement noises: $\quad v_{k}^{i}=y_{k}-G_{k}\left(x_{k}^{i}\right)$

- Update the weights: $\quad \omega_{k}^{i \prime}=\omega_{k-1}^{i} f_{v}\left(v_{k}^{i}\right)$

- Normalize the weights: $\quad \omega_{k}^{i}=\omega_{k}^{i \prime} / \sum_{i=1}^{m} \omega_{k}^{i \prime}$

- Construct the posterior density: $\quad f_{x}\left(x_{k} \mid Y_{k}\right) \approx \sum_{i=1}^{m} \omega_{k}^{i} K_{\mathrm{f}}\left(x_{k}-x_{k}^{i}\right)$

- Draw the new particles: $\quad\left(x_{k}^{i}, \omega_{k}^{i}=1 / m\right) \sim f_{x}\left(x_{k} \mid Y_{k}\right)$

\subsubsection{Filtering the Performance Indicators}

There are fifteen time segments between consecutive compressor washes and each segment has a particular trend. By examining different functions, combination of linear logarithmic transform and an affine function is obtained to model the indicators in each segment

$$
F(t)=\alpha_{1}+\alpha_{2} \ln \left(\alpha_{3} t+\alpha_{4}\right)+\alpha_{5} t .
$$

For $E H$ and $E R$ indicators, the coefficients are found through least squares best fit in each segment and there is a modeling error at each time step: $\left(t_{k^{\prime}}\right)=x\left(t_{k^{\prime}}\right)-F\left(t_{k^{\prime}}\right)$. Assuming $\Delta t$ the time length between the steps, the state model similar to Equation (5.22) is

$$
x\left(t_{k^{\prime}}\right)=x\left(t_{k^{\prime}-1}\right)+\alpha_{2} \ln \left(1+\alpha_{3} \Delta t /\left(\alpha_{3} t_{k^{\prime}-1}+\alpha_{4}\right)\right)+\alpha_{5} \Delta t+\tau\left(t_{k^{\prime}}\right),
$$

where $\tau\left(t_{k^{\prime}}\right)$ is the representative of the process noise at each time step with the following value:

$$
\tau\left(t_{k^{\prime}}\right)=\varepsilon\left(t_{k^{\prime}}\right)-\varepsilon\left(t_{k^{\prime}-1}\right) .
$$

There are $n-1$ samples for the process noise found by Equation (5.29). In the state equation of the filter, the random noise of the process is therefore a draw from the consequent distribution 


$$
f_{\tau}\left(\tau_{k}\right) \approx \sum_{j=2}^{n} K_{\mathrm{h}}\left(\tau-\tau\left(t_{j}\right)\right) .
$$

In the constructed framework, the indicators are considered both the measurement signal and the state of the system. To visualize the performance of RPF state estimation, Figure 5.3 depicts the original signals, the prediction models and the filtered signals in selected windows.

(a)

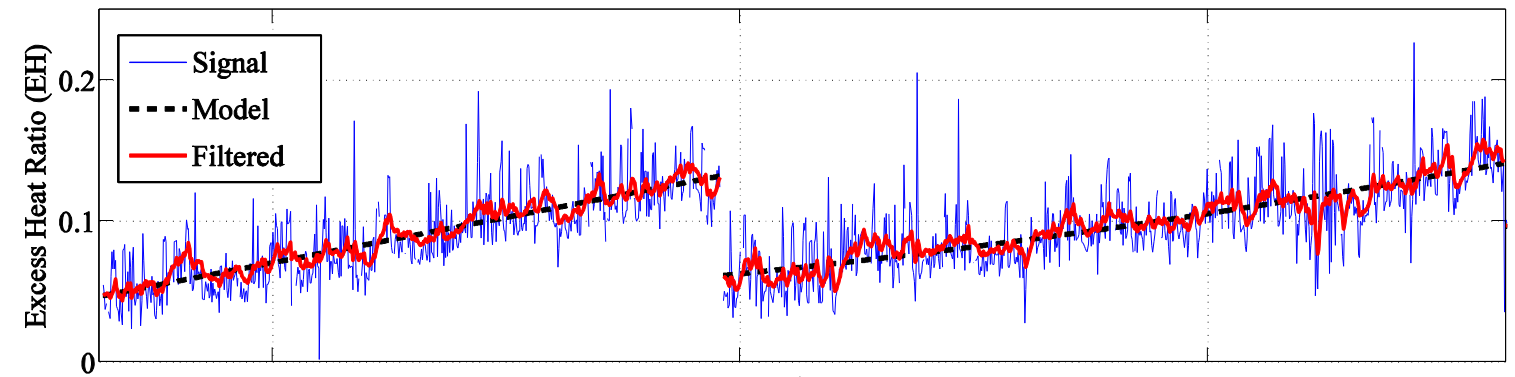

(b)

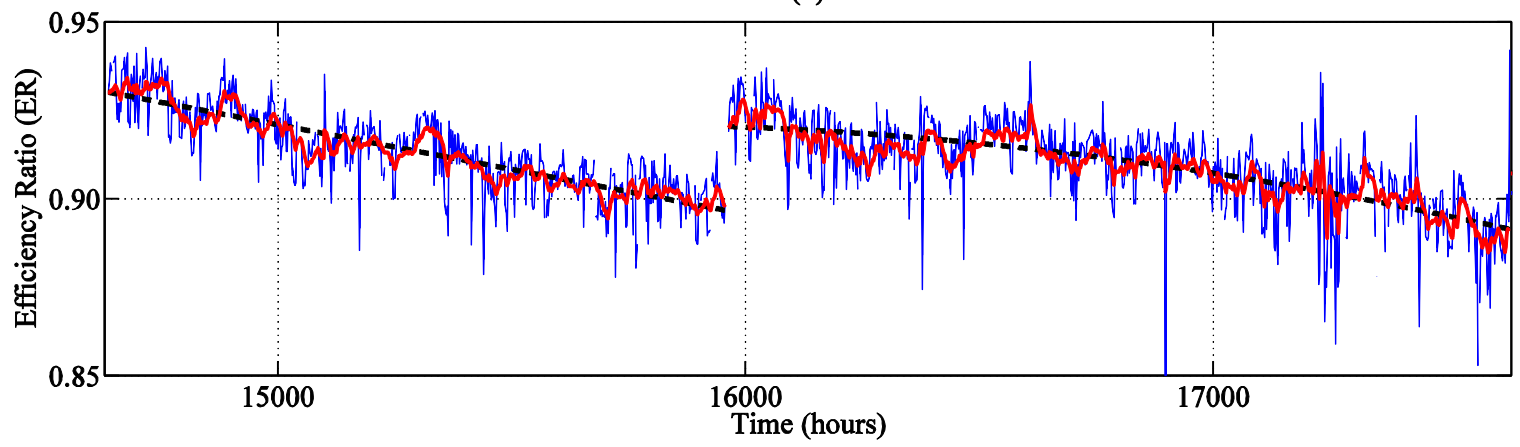

Figure 5.3. RPF state estimation (red) on the performance indicator signals (blue): (a) $E H$ performance indicator, and (b) $E R$ performance indicator.

\subsection{Effective Factors on the Rate of Performance Deterioration}

As per section 5.2.1, three ambient conditions $\left(T_{C i}, P_{C i}, S H_{C i}\right)$ and three operating parameters $(N, P W, E G T)$ are used to calculate the performance indicators at each time step. It was shown that the short-term performance deterioration is the dominant part of the performance indicator [97]. As a result, the indicators can be effectively used to quantify the compressor fouling, and the corresponding variation rates of the indicators will indicate the rate of fouling.

Studies have shown a positive correlation between the fouling rate and the GTE 
power [159]. It can be explained by ingestion of more particulates suspended in larger volume of airflow, when the gas turbine runs with a higher power. Some qualitative reports explain a twofold relation between the rate of condensed humidity of the intake air and the fouling rate, i.e., with a low rate of humidity condensation, combination of water droplets with the ingested particles deposit on the blades and vanes, which accelerates the fouling rate. With increase of the condensation rate beyond a certain level, the extra water will naturally wash the surfaces and decrease the fouling rate [157]. Two other effective factors in fouling rate are the air pollution and the filter design [141], which are not recorded in the available logged data used in this work. Consequently, this study on the effective factors on the fouling rate is limited to the ambient conditions and the GTE operating parameters.

\subsubsection{Effects of Power and Condensed Humidity}

The GTE power $(P W)$, the air mass flow $\left(W_{C i}\right)$ and the rate of condensed humidity in the intake duct $\left(W_{c o n}\right)$ are all effective in the rate of short-term performance deterioration. To quantify the rate of performance deterioration, the time derivative of the filtered signals of the indicators is represented as:

$$
\dot{E} H_{k} \approx\left(E H_{k+1}-E H_{k}\right) / \Delta t,
$$

and similarly for $E R_{k}$. To investigate the relation between the above mentioned factors and the variation rate of the indicators, the rates are plotted versus $P W-W_{\text {con }}$ in Figure 5.4. As appears, the plotted points are scattered and do not lie on a single surface in either figure. In other words, there are many cases with almost equal operating conditions, i.e., $P W-W_{\text {con }}$, which do not result in the same variation rates for the indicators. Examining the operating conditions with $W_{C i}-W_{c o n}$ pairs provide similar results. Consequently, the variation rates of the performance indicators depend on other factors at the same time. To tackle this problem, a state function for each indicator is suggested, which takes into account the past state to predict the next state 


$$
\begin{aligned}
& E H_{k+1}=F_{E H}\left(E H_{k}, P W_{k}, W_{\text {con }_{k}}\right), \\
& E R_{k+1}=F_{E R}\left(E R_{k}, P W_{k}, W_{\text {con }_{k}}\right) .
\end{aligned}
$$

Alternatively, one may use the air mass flow $W_{C i}$ instead of the GTE power for the state functions

$$
\begin{aligned}
& E H_{k+1}=F_{E H}\left(E H_{k}, W_{C i_{k}}, W_{\text {con }_{k}}\right), \\
& E R_{k+1}=F_{E R}\left(E R_{k}, W_{C i_{k}}, W_{\text {con }_{k}}\right) .
\end{aligned}
$$

(a)

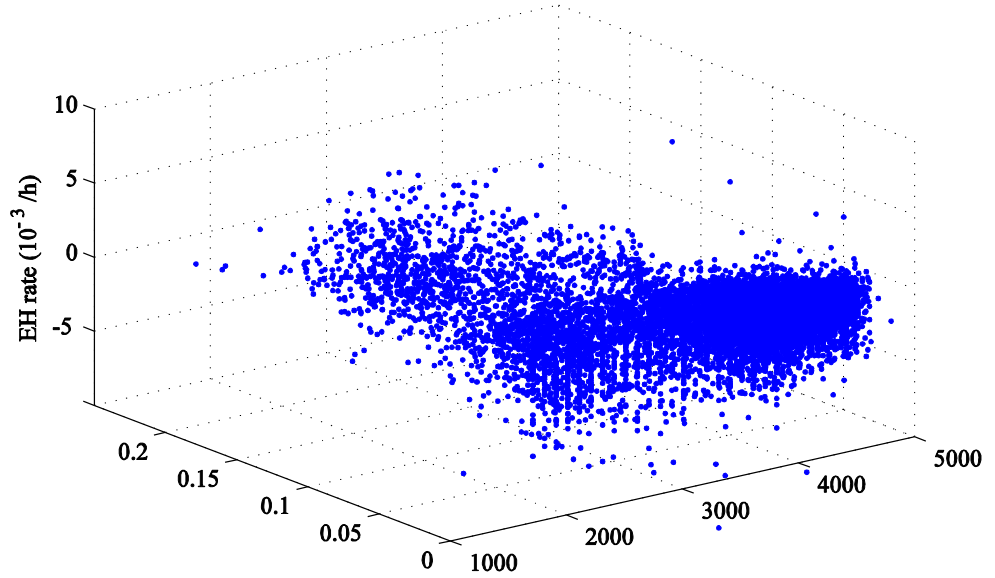

(b)

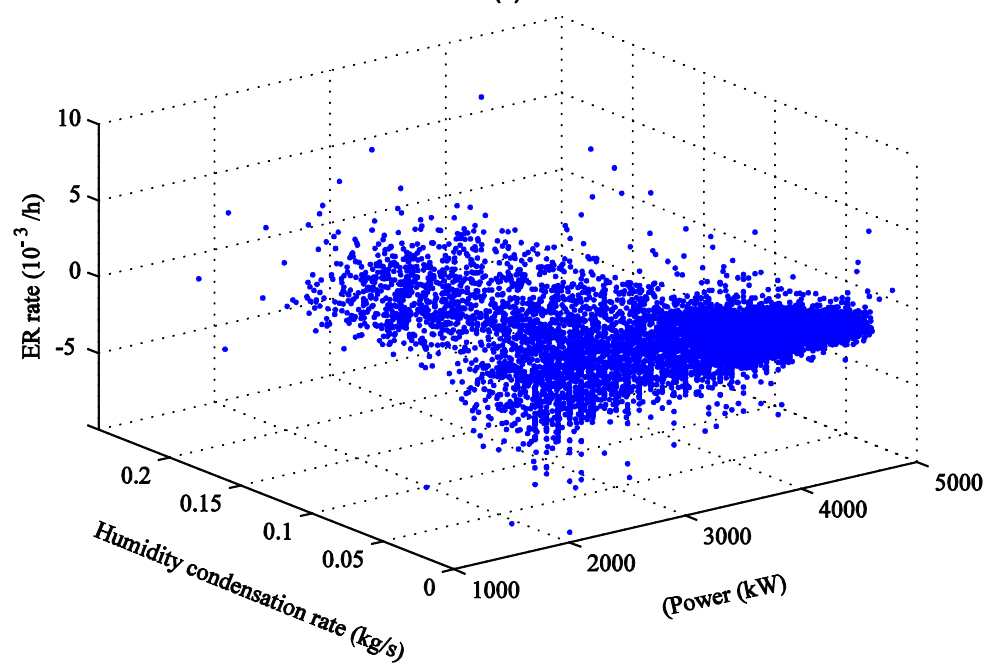

Figure 5.4. Variation rates of the performance indicators do not constitute functions over $P W-W_{c o n}$ space: (a) variation rate of $E H$, and (b) variation rate of $E R$. 
There are over 11,000 time step data available from the GTE to build the above model. Considering the step number $k$, there are four variables in each of the state equations, which makes it hard to visualize the effects of the inputs on the outputs. An effective practice to establish data-driven models on vector time series with large number of time steps is implementation of machine learning frameworks.

\subsubsection{Data-Driven Model Construction}

Effects of the inputs on the outputs in Equations (5.32) through (5.34) are only known qualitatively. This calls for system identification techniques that can extract unknown model structures. For such problems, various techniques have been practiced, ranging from multivariate polynomial regression to more advanced machine learning techniques, e.g.; artificial neural network (ANN), and adaptive neuro-fuzzy inference system (ANFIS) [160-162]. For this modeling problem, there are two main motivations to employ ANFIS data-driven scheme. Firstly, the number of membership functions can be adjusted for each input, based on the complexity expected from pertaining behavior. The adjustment is devised based on the expert knowledge. This is not the case for ANN, where the internal units are opaque to the users. Secondly, during several practices, ANFIS showed excellent repeatability when trained with a set of vector time series. The experiments with ANN showed much less repeatability when trained on the same set of data, which complies with some reported literature [163].

\subsection{Performance Deterioration Modeling Using ANFIS}

In early 1990s, Jang developed ANFIS, which combines the qualitative approach of the fuzzy models and the adaptive capability of the neural network to optimize the internal coefficients of the system to achieve the desired performance [70]. If sufficient input-output data are available, ANFIS adapts itself through the training phase without a need to the expert knowledge required to design the conventional fuzzy systems. In sections 5.5.1 through 5.5.3, an ANFIS framework is presented for system identification and model extraction. 


\subsubsection{Building ANFIS Structure and Training}

Assuming the state function in Equation (5.32) at time step $k$, the model receives four inputs, i.e., $E H_{k}, P W_{k}, W_{c o n}$ and the count of the time step $k$, which represents the time step number after the compressor wash. The model predicts the next step state $E H_{k+1}$ as the output. Figure 5.5 shows the ANFIS structure used to estimate the mentioned function. A first-order Sugeno fuzzy model [164] is used for this structure.

- The first layer and the last layer correspond to the inputs and the output respectively.

- The input membership functions are in the second layer. In this layer, generalized bellshaped membership functions are used. As mentioned in section 5.4.2., the number of membership functions is a design choice, which is based on the expected complexity of the input effects on the output. Considering the highly nonlinear effects of the condensed humidity mass flow on the performance deterioration rate [157], three membership functions are assigned for this input. For the other inputs, two membership functions are employed.

- The third layer corresponds to the fuzzy rules, assigned for all combinations of the membership functions. In this structure there are $2 \times 2 \times 3 \times 2=24$ linear fuzzy rules.

- The fourth layer provides the normalized weights for the rules. The weights are resulted from multiplication of corresponding input membership functions.

- The output of the fuzzy rules from layer three are multiplied in the corresponding normalized weights from layer four. The results are then summed up in layer five to provide ANFIS output.

Once the model architecture is constructed, the training set of data is introduced to the model in order to tune the variable coefficients of the membership functions and fuzzy rules in layers two and three respectively. Because the model is designed to estimate the short-term performance deterioration, two adjustments are made in the training data set:

1) The count of the time step $k$ is set back to "one" after each compressor wash. Table 5.2 shows the compressor wash times and the corresponding time step counts.

2) The fouling effect is majorly removed by compressor washing, and the value of the performance indicator after each wash is only attributed to the long-term degradation. To eliminate this long-term deterioration effect from the performance indicator, the 
average of the indicator in the first three days (36 time steps) after each wash is subtracted from the value of the performance indicator calculated for an entire segment.

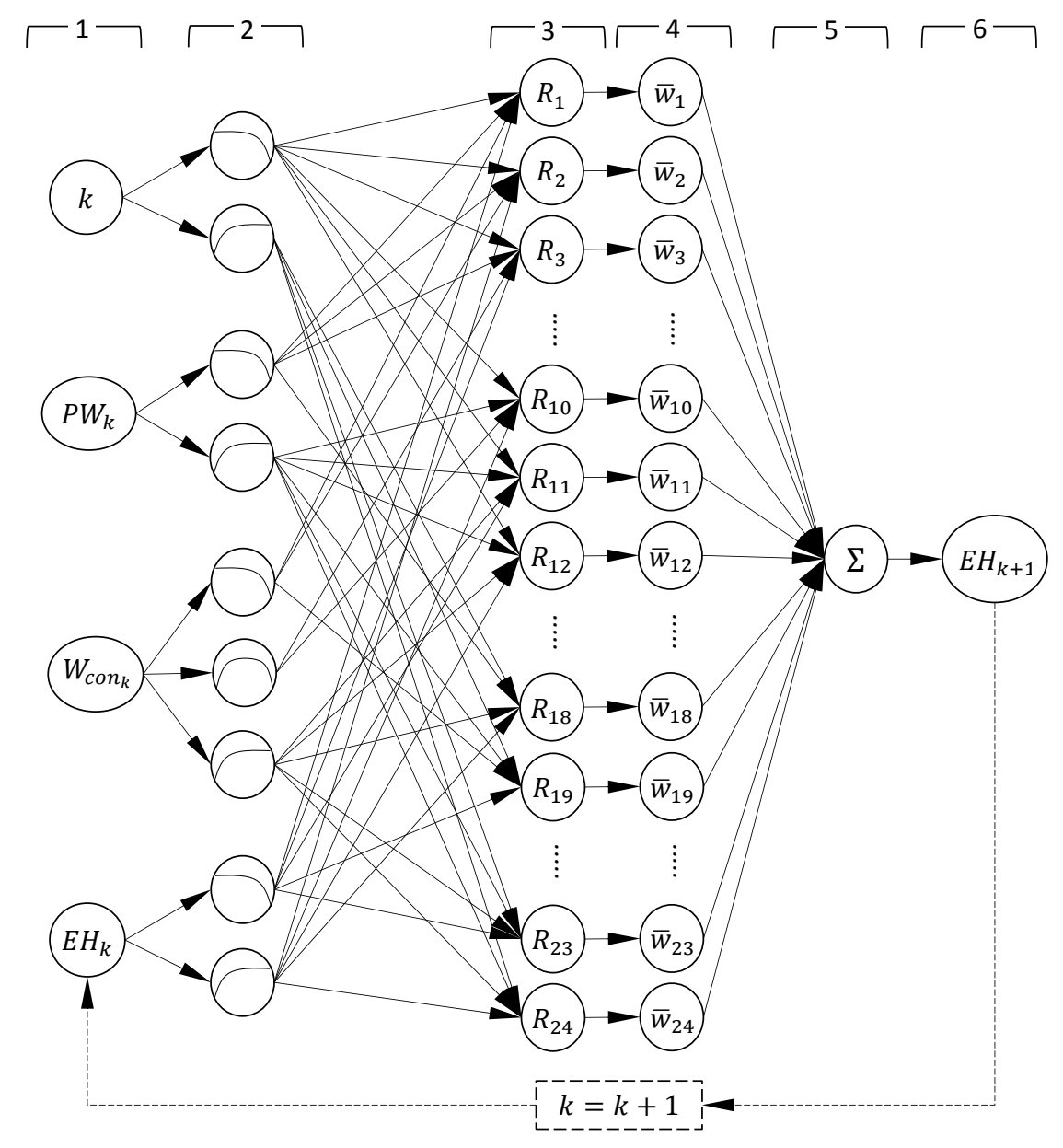

Figure 5.5. ANFIS structure for system identification. Dashed line shows the use of output as input of the next step for profile simulation.

Hybrid learning algorithm is used as the training algorithm for the model, which applies a combination of the backpropagation gradient descent method and the leastsquares method. To prevent over fitting to the training data, $10 \%$ of the data were separated for model validation, i.e., the training iterations were stopped when the modeling error on the validation data reached a plateau. Figure 5.6 shows the data points 
in $P W-W_{c o n}-E H$ and $P W-W_{c o n}-E R$ spaces and the corresponding predictions by the ANFIS model. With respect to standard deviation of the corresponding indicator, the normalized modeling error for $E H$ is $2.29 \times 10^{-4}$, and for $E R$ is $3.60 \times 10^{-4}$ that shows an acceptable modeling accuracy for the ANFIS models.

\subsubsection{Running the ANFIS Model}

By training the constructed ANFIS, the model can estimate the next step state $E H_{k+1}$ at any step $k$, given $E H_{k}, P W_{k}$ and $W_{c o n}$. To run the model to estimate the performance profiles under different operating conditions, the following steps are taken:

1) $P W$ and $W_{c o n}$ are kept constant for each profile, to analyze the performance deterioration under a given operating condition.

2) Initial value of the indicator is set to zero, i.e., $E H_{1}=0$, to study the performance deterioration beginning right after a compressor wash.

3) The output of the $k^{\text {th }}$ step is taken as the input of the fourth node in step $k+1$. This is shown with dashed lines in Figure 5.5.

4) The model is run up to 370 time steps, which simulates 740 hours of operation after the compressor wash. The step number is limited considering the shortest segment for which the training data is available, as given in Table 5.2.

Once a performance profile is acquired, the same steps will be repeated for other $P W$ and $W_{\text {con }}$ values within the domain of available training data, i.e., $2000 \mathrm{~kW} \leq P W \leq$ $4500 \mathrm{~kW}$, and, $0 \mathrm{~kg} / \mathrm{s} \leq W_{\text {con }} \leq 0.2 \mathrm{~kg} / \mathrm{s}$. As it is seen in Figure 5.6(c), at some powers, the humidity condensation rate does not fully cover the corresponding domain and no training data are available for those operating conditions. Consequently, the model cannot be used for performance prediction in such operating conditions. 
(a)

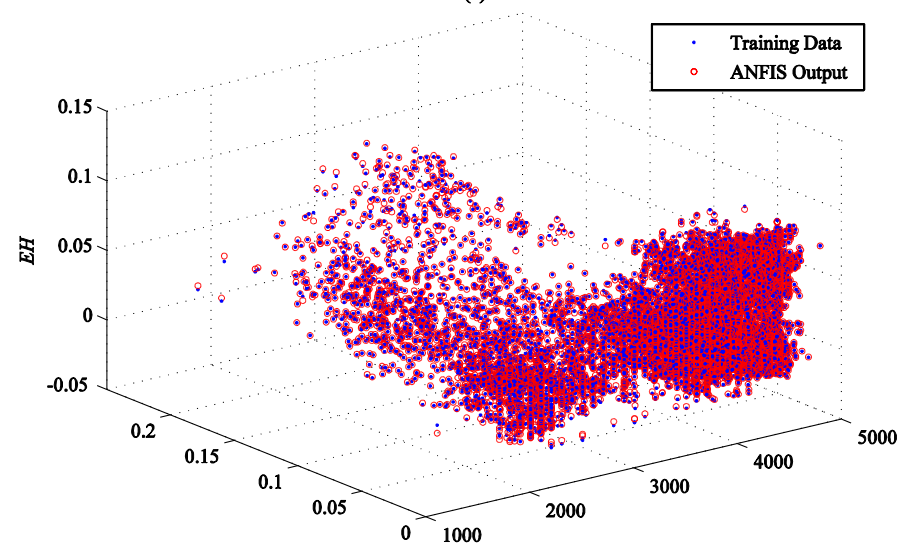

(b)

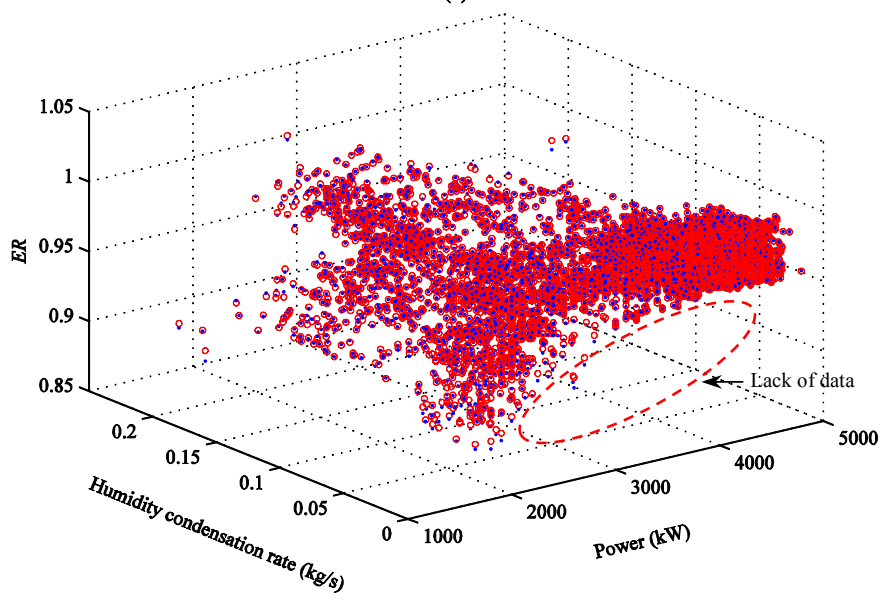

(c)

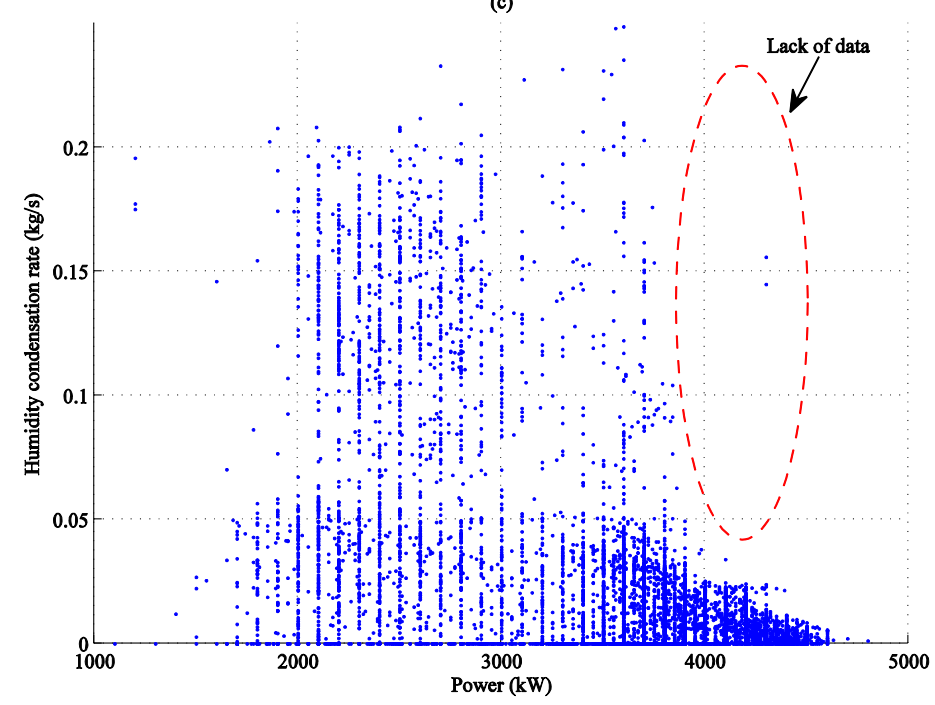

Figure 5.6. Training data points and the result of ANFIS model prediction: (a) $E H$ data and prediction, (b) ER data and prediction, and (c) scarcity of data points in some regions of the domain. 


\subsubsection{Simulated Profiles}

The processes in sections 5.5.1. and 5.5.2. can similarly be repeated to construct ANFIS models based on state functions in Equations (5.33) to (5.35). In this section, the results of all trained ANFIS models are analyzed.

\subsubsection{1 $\quad E H$ Variations Given $P W$ and $W_{c o n}$ :}

The corresponding trained ANFIS model is run at constant $P W$ and $W_{\text {con }}$ to simulate EH variations. Figure 5.7(a) depicts the extracted behavior of the system through the simulation within the operating domain. Each curve shows the variations of $E H$ at constant $P W$ and $W_{\text {con }}$ condition. As the power exceeds $3000 \mathrm{~kW}$, the available data points become limited to less humidity condensation rates, and the ANFIS model cannot be used for such regions as stated earlier. That is why for higher power levels, simulated variations of $E H$ are limited to drier conditions. It is seen that regardless of the power level, there is a monotonic performance deterioration when the intake air is fully dry. By the growth of humidity condensation rate at any power level, the performance deterioration accelerates, in comparison with the dry condition. However, with the higher humidity levels, the growth of EH tapers off and an inverse trend appears in the simulated $E H$.

At a fixed power like $2400 \mathrm{~kW}$ with an abundance of the training data points throughout the entire humidity condensation rates, Figure 5.7(b) shows the resulting $E H$ variations. When the inlet air is quite dry, $E H$ increases slowly and steadily, and reaches to 0.007 in 740 hours. With higher humidity condensation rates, the performance deterioration speeds up, such that under $W_{c o n}=0.025 \mathrm{~kg} / \mathrm{s}, E H$ reaches to 0.04 in 740 hours, which faster than that of the dry conditions. For performance deterioration profile under $W_{\text {con }}=0.050 \mathrm{~kg} / \mathrm{s}$, within the first 200 hours, $E H$ exceeds the domain of the available training data points for Equation (5.32). Therefore the simulation results of the ANFIS model will be no more valid, and it is truncated after $E H=0.04$. Beyond $W_{\text {con }}=$ $0.075 \mathrm{~kg} / \mathrm{s}$, two common behaviors are observable in the simulated profiles; the rate of performance deterioration is rapid in the beginning, such that $E H$ passes 0.01 within the 
first 25 to 70 hours. After a certain time, depending on the humidity condensation rate, the performance does not deteriorate any further, and gradually and limitedly improves. With humidity condensation rates over $0.100 \mathrm{~kg} / \mathrm{s}$, the performance deterioration reaches to its maximum within the first 200 hours, after which an improvement takes place that can be accounted to a phenomena known as self-cleaning due to natural washing [141].
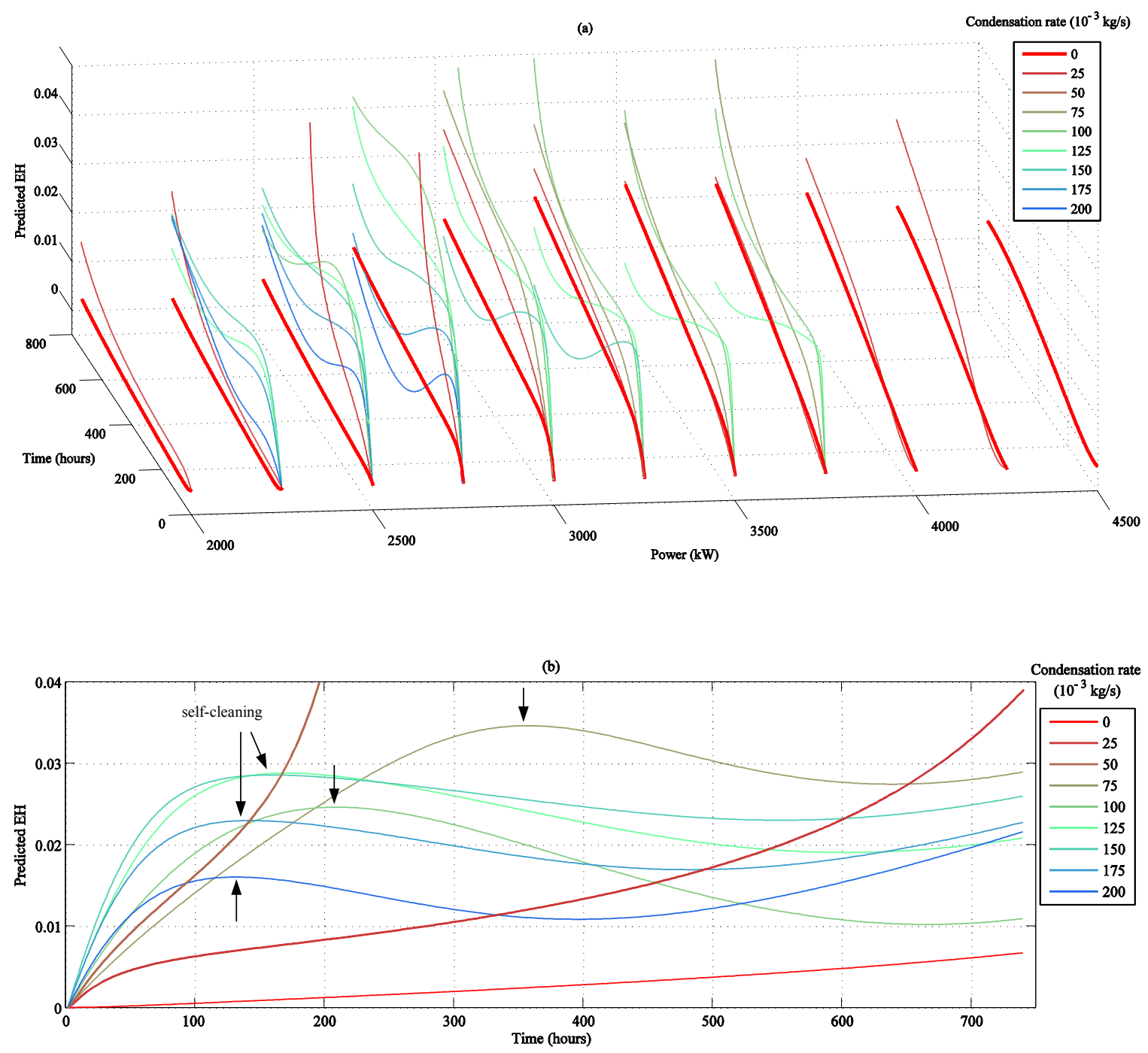

Figure 5.7. ANFIS model simulated profiles for $E H$ : (a) $E H$ profiles at constant power and humidity condensation rates, and (b) $E H$ profiles at $P W=2400 \mathrm{~kW}$. 
Comparison between the profiles with different condensation rates provides the two following key results: 1) Although in the beginning of the profiles, the performance deterioration accelerates with the increment of the humidity condensation rate, beyond the condensation rate $W_{\text {con }}=0.150 \mathrm{~kg} / \mathrm{s}$, the early deterioration slows down. 2) When the condensation rate exceeds $W_{\text {con }}=0.150 \mathrm{~kg} / \mathrm{s}$, the self-cleaning takes place at lower $E H$ levels, e.g., it happens at $E H \approx 0.03$ for $W_{\text {con }}=0.150 \mathrm{~kg} / \mathrm{s}$, while it hardly reaches to 0.016 when $W_{\text {con }}=0.200 \mathrm{~kg} / \mathrm{s}$.

\subsubsection{ER Variations Given $P W$ and $W_{\text {con }}$ :}

The ANFIS model is trained on the corresponding data points shown in Figure 5.6(b). Simulation results are shown in Figure 5.8 for $P W=3500 \mathrm{~kW}$, where there are relatively more training data points. Here, the smallest deterioration rates correspond to the condensation rate of $0.025 \mathrm{~kg} / \mathrm{s}$. The performance deterioration rate gets faster by the increment of the humidity condensation rate, however, by exceeding from $W_{\text {con }}=0.100$ $\mathrm{kg} / \mathrm{s}$, the deterioration rate slows down, and with the further condensation rates, an improvement due to self-cleaning could be observed in the profiles. In comparison with $E H$, the improvement seems take place earlier within the first 50 hours, and as the humidity condensation rate exceeds $0.100 \mathrm{~kg} / \mathrm{s}$, the performance indicator becomes limited to larger $E R$ values. For instance, for $W_{\text {con }}=0.125 \mathrm{~kg} / \mathrm{s}$, the minimum $E R$ value reaches to 0.983 , whilst with $W_{\text {con }}=0.200 \mathrm{~kg} / \mathrm{s}$, the $E R$ value stays above 0.987 . This means, with the higher humidity condensation rates, there will be more improvement in the performance.

Figure 5.6(b) shows the distribution of the training data is not as even as that of $E H$ in Figure 5.6(a), and the data points for $P W>3000 \mathrm{~kW}$ are accumulated above $E R \geq 0.95$. This lack of diversity leads to imperfect training of ANFIS model, and consequently in the resulting simulation for the first 740 hours, $E R$ variations are bounded to the values above 0.97 . 


\subsubsection{3 $\quad E H$ Variations Given $W_{C i}$ and $W_{c o n}$ :}

Intake air flow carries the unfiltered particulates, what contributes to the compressor fouling and leads to the short-term performance deterioration. This effect is expressed by Equation (5.33), and an ANFIS model is structured and trained with the corresponding data points. Figure 5.9 is resulted from the simulation by running the model in a region of abundant data points, i.e., $W_{C i}=16.75 \mathrm{~kg} / \mathrm{s}$. The results are much similar to the case A, i.e., the rate of performance deterioration is minimal with the dry air and it shows an increasing trend with the increase in the condensation rate. Similarly, at the beginning of the profiles, the rate of performance deterioration increases with the condensation rate, however for the condensation rates over $W_{\text {con }}=0.150 \mathrm{~kg} / \mathrm{s}$, i.e., $\sim 0.9 \%$ of the intake mass flow, the early performance deterioration slows down, and at the same time, the improvement due to self-cleaning takes place at smaller values of $E H$. For instance, for $W_{\text {con }}=0.150 \mathrm{~kg} / \mathrm{s}$, the performance improvement due to self-cleaning takes place after 230 hours, when $E H$ has reached to 0.32 , while for $W_{c o n}=0.200 \mathrm{~kg} / \mathrm{s}$, it occurs after 150 hours, when $E H$ is 0.22 .

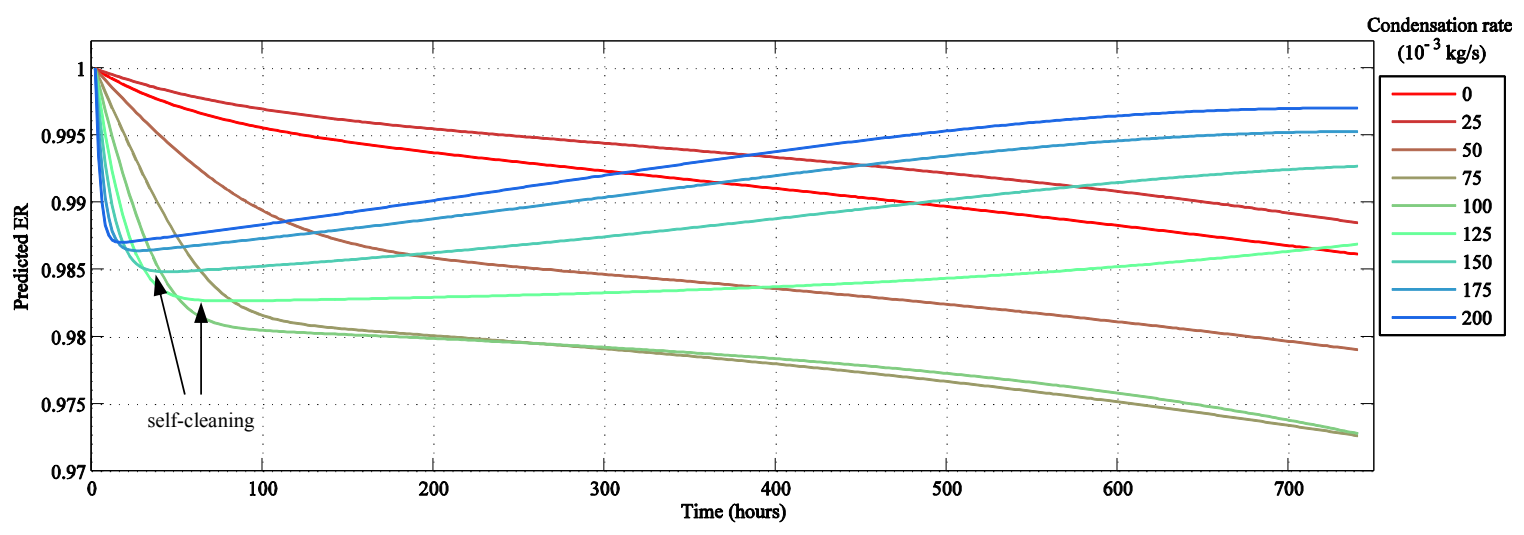

Figure 5.8. $E R$ simulated profiles at $P W=3500 \mathrm{~kW}$. 


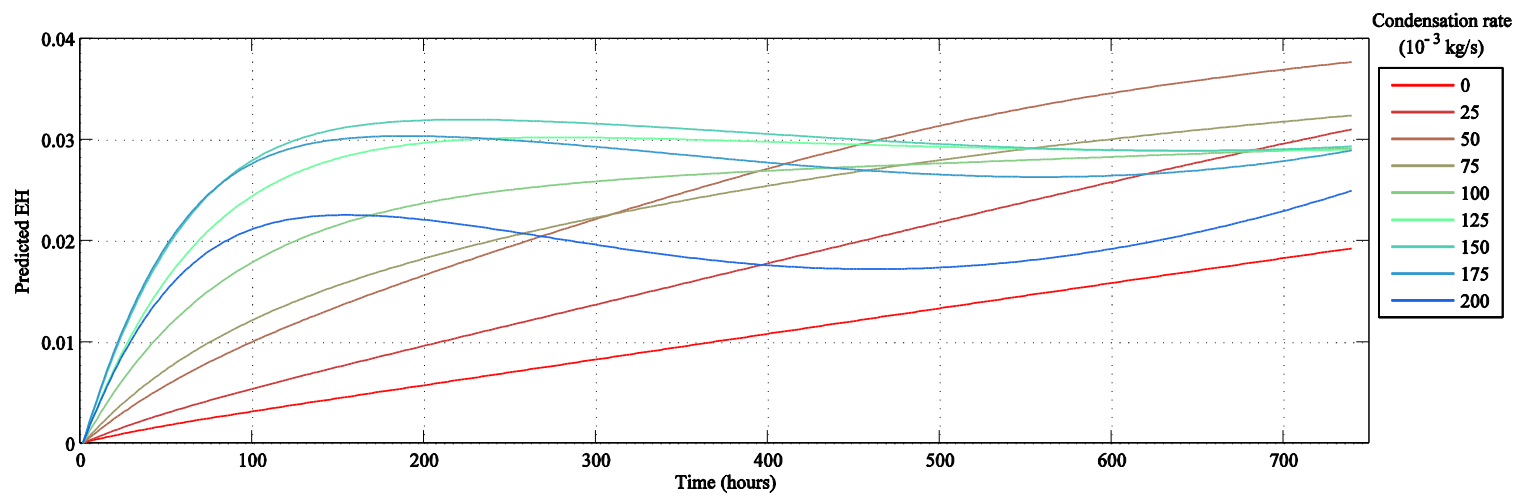

Figure 5.9. $E H$ simulated profiles at $W_{C i}=16.75 \mathrm{~kg} / \mathrm{s}$.

\subsubsection{ER Variations Given $W_{C i}$ and $W_{c o n}$ :}

Distribution of the training data points for the ANFIS model using Equation (5.35) is shown in Figure 5.10. There are large gaps in the data space, where there is no data for training, while most of the data points are concentrated in a particular region. Hence, the resulting simulation does not provide reliable output for the corresponding ANFIS model.

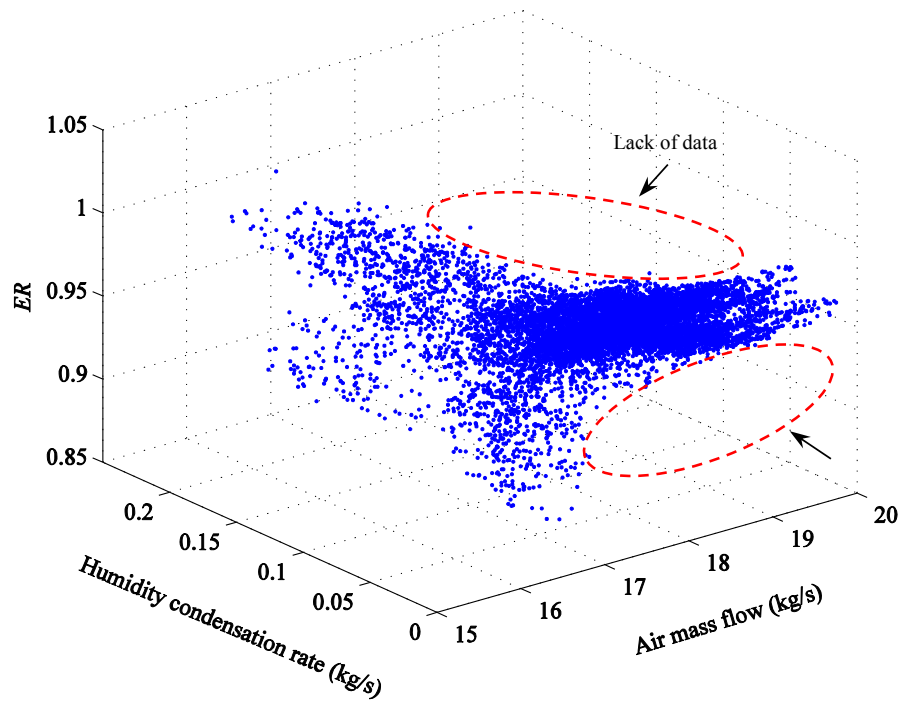

Figure 5.10. Lack of training data points for $E R$ simulation with $W_{C i}-W_{c o n}$ data. 


\subsection{Summary}

Effects of the ambient and the operating conditions on the short-term performance variations of a GTE were studied in this chapter. To quantify the GTE performance, two physics-based performance indicators were obtained from the previous chapters. The effective parameters in the GTE performance deterioration were further investigated and the rate of humidity condensation, which is a known factor in the compressor fouling was then calculated at each time step. To estimate the health state of the system, a particle filter framework was devised to filter $E H$ signal. The output of the filter along with the GTE power and the rate of humidity condensation were considered for training an ANFIS model in order to extract the system behavior. The ANFIS model was then run under several scenarios of constant power - constant humidity condensation rate. The same procedure was repeated for constant intake mass flow - constant humidity condensation rate scenarios, and then repeated for $E R$ indicator. The resulting simulations clearly revealed the GTE deterioration behavior influenced by the mentioned ambient and the operating conditions. $E H$ exhibited superiority in comparison with $E R$ in that, the resulting simulation profiles could reasonably be explained by physics of the phenomena. The rate of humidity condensation shows a dual effect behavior in the short-term performance deterioration, i.e., increasing the humidity condensation rate may deteriorate or improve the trend of performance variation, depending on the conditions. The slowest performance deterioration takes place in the dry air. With the increment of the humidity condensation, the rate of deterioration increases in the beginning and gradually slows down. The GTE performance deteriorates the fastest, when the humidity condensation rate reaches to $0.150 \mathrm{~kg} / \mathrm{s}$, i.e., $\sim 0.9 \%$ of the intake mass flow, after which the increase of the humidity condensation rate causes slower performance deterioration that can be accounted to the self-cleaning phenomenon.

The developed tool in this research and the corresponding results can be beneficial for two major applications; 1) the short-term performance variations and the expected health state of the GTE can be predicted by processing the ambient and the operating records; and 2) the optimum humidity condensation rate that leads to self-cleaning of the compressor can be estimated. The required humidity for such operating conditions can be accommodated by the inlet air wet cooler, available in many of the GTEs. 


\section{Chapter 6: Sequential State Estimation of Nonlinear/Non-Gaussian Systems with Stochastic Input for Turbine Degradation Monitoring}

\subsection{Overview}

In the hot section of the gas turbine engines (GTEs), the components work in harsh operating conditions and are prone to various degradation mechanisms. Such mechanisms lead to different fault modes in the components. The dominant fault modes in the nozzle and the turbine section are known as: a) increase of the surface roughness in the nozzle vanes and the turbine blades [165], b) increase of the tip clearance in the turbine, and c) material removal and deformation of the profile [166, 167]. The fault modes manifest their symptoms as deviations in the turbine performance map, i.e., under a constant operating condition, all the fault modes lead to loss of isentropic efficiency in the turbine, whereas the tip clearance and material removal from the profile lead to increase of the mass flow [168-171]. When the GTE is running, the only directly measurable fault is the tip clearance using advanced instrumentation, while the other two fault modes remain inaccessible for direct measurement. Intensity of the faults therefore should be assessed indirectly through their symptoms in deviating the turbine performance, namely, loss of isentropic efficiency and rise of the mass flow. The fault parameters (the symptoms) are the parameters of the thermodynamic model of the GTE, and they should be estimated using the available measurements of the system $[13,105]$. The GTE receives timevarying input, i.e., the control variables and the ambient conditions, and it generates the output. The corresponding signals of the input / output are always noisy, while the noise structure is statistically unknown in most cases. From this prospect, GTE degradation phenomenon translates into a system identification problem, where the system internal parameters are the evolving state of the dynamical system with the following specifications:

- $\quad$ The fault symptoms are considered the parameters of the degrading system.

- Measurement signals on the input / output of the system are accessible.

- $\quad$ Both the input and the output signals are noise contaminated.

- $\quad$ Noise structure is statistically unknown. 
A dynamical system in its general form receives time-variant input; however, inclusion of the input in PF scheme has just been noted in some recent works. For instance, Won et al used PF for orientation estimation with deterministic system input [172], and Gustafsson assumed the control input to be known in applying PF for positioning [173]. In practice, the inputs of the systems are noisy and treating them as deterministic variables is not accurate. Consequently, there will be a need to state estimation frameworks for nonlinear/non-Gaussian dynamical systems where the system input is statistically stochastic.

\subsection{Sequential State Estimation for Nonlinear System with Stochastic Input (NSI)}

Nonlinear/non-Gaussian dynamical systems take no closed form solution for state estimation. Profiting from the available computational resources, PFs simulate Monte Carlo sequential method by a set of representative scenarios to estimate the posterior distribution of the evolving state [89]. When the dynamical systems receive stochastic inputs, PF technique needs to be reformulated to take into account the input effects.

\subsubsection{Formulation of NSI State Evolution}

In an NSI with first order Markov process, at a time-step $k$, the state vector $x_{k}$ is a function of the previous step state $x_{k-1}$ and the system input $u_{k} . F$ is the state model with a local error of $\tau_{k}$, caused by uncertainty of the state model with a known probability density of $f_{x}\left(x_{k} \mid x_{k-1}, u_{k}\right)$.

$$
x_{k}=F\left(x_{k-1}, u_{k}\right)+\tau_{k}
$$

The output vector $y_{k}$ is a function of the same step state $x_{k}$ and the system input $u_{k} . G$ is the measurement model with a known uncertainty marginal distribution of $f_{y}\left(y_{k} \mid x_{k}, u_{k}\right)$ that creates a local error of $v_{k}$.

$$
y_{k}=G\left(x_{k}, u_{k}\right)+v_{k}
$$

Assuming that the initial density of the state $f_{x}\left(x_{0}\right)$ and the density of the input $f_{u}\left(u_{k}\right)$ are both known, and given the history of the input $Y_{k} \triangleq\left\{y_{1}, y_{2}, \ldots, y_{k}\right\}$ and the output $U_{k} \triangleq\left\{u_{1}, u_{2}, \ldots, u_{k}\right\}$ are available, we can calculate the prior density of the state at step $k$ 
from the posterior density at the previous step $f_{x}\left(x_{k-1} \mid Y_{k-1}, U_{k-1}\right)$,

$$
f_{x}\left(x_{k} \mid Y_{k-1}, U_{k-1}\right)=\int f_{x}\left(x_{k} \mid x_{k-1}\right) f_{x}\left(x_{k-1} \mid Y_{k-1}, U_{k-1}\right) d x_{k-1},
$$

where the probability transition density due to independence of $x_{k-1}$ and $u_{k}$ is

$$
f_{x}\left(x_{k} \mid x_{k-1}\right)=\int f_{x}\left(x_{k} \mid x_{k-1}, u_{k}\right) f_{u}\left(u_{k}\right) d u_{k}
$$

The marginal filtering density is provided by Bayes' theorem correspondingly,

$$
f_{x}\left(x_{k} \mid Y_{k}, U_{k}\right)=\frac{f_{y, u}\left(y_{k}, u_{k} \mid x_{k}\right) f_{x}\left(x_{k} \mid Y_{k-1}, U_{k-1}\right)}{f_{y, u}\left(y_{k}, u_{k} \mid Y_{k-1}, U_{k-1}\right)}
$$

where, the denominator is

$$
f_{y, u}\left(y_{k}, u_{k} \mid Y_{k-1}, U_{k-1}\right)=\int f_{y, u}\left(y_{k}, u_{k} \mid x_{k}\right) f_{x}\left(x_{k} \mid Y_{k-1}, U_{k-1}\right) d x_{k} .
$$

It can be shown that

$$
f_{y, u}\left(y_{k}, u_{k} \mid x_{k}\right)=\frac{f_{y}\left(y_{k} \mid x_{k}, u_{k}\right)}{f_{x}\left(x_{k}\right)} \int f_{x}\left(x_{k} \mid x_{k-1}, u_{k}\right) f_{x}\left(x_{k-1}\right) d x_{k-1},
$$

and $f_{x}\left(x_{k}\right)$ can be sequentially found by integrating from its transition density in Equation (6.4),

$$
f_{x}\left(x_{k}\right)=\int f_{x}\left(x_{k} \mid x_{k-1}\right) f_{x}\left(x_{k-1}\right) d x_{k-1} .
$$

Equations (6.3) through (6.8) formulate the Bayesian recursive estimation problem for NSIs described by Equations (6.1) and (6.2).

\subsubsection{Application of PF}

The posterior density $f_{x}\left(x_{k} \mid Y_{k}, U_{k}\right)$ in PF techniques is numerically represented by $\mathrm{m}$ sample scenarios, i.e.: particles $x_{k}^{1}, \ldots, x_{k}^{m}$ along with their corresponding assigned weights $\omega_{k}^{1}, \ldots, \omega_{k}^{m}$,

$$
f_{x}\left(x_{k} \mid Y_{k}, U_{k}\right) \approx \sum_{i=1}^{m} \omega_{k}^{i} \delta\left(x_{k}-x_{k}^{i}\right), \quad\left(\sum_{i=1}^{m} \omega_{k}^{i}=1\right)
$$

where $\delta$ is Dirac delta function The accuracy of the approximation increases as $m \rightarrow \infty$ [152]. As soon as a new output $y_{k}$ becomes observable with a new input $u_{k}$, the weights of the particles can be updated, given an importance density $g_{x}\left(x_{k} \mid x_{k-1}^{i}, y_{k}, u_{k}\right)$ is 
provided,

$$
\omega_{k}^{i} \propto \omega_{k-1}^{i} \frac{f_{y, u}\left(y_{k}, u_{k} \mid x_{k}^{i}\right) f_{x}\left(x_{k}^{i} \mid x_{k-1}^{i}\right)}{g_{x}\left(x_{k}^{i} \mid x_{k-1}^{i}, y_{k}, u_{k}\right)} .
$$

To increase the effectiveness of the samples, we minimize the variance of the weights, which leads to the optimal importance density [153],

$$
g_{x}\left(x_{k} \mid x_{k-1}^{i}, y_{k}, u_{k}\right)=\frac{f_{y, u}\left(y_{k}, u_{k} \mid x_{k}, x_{k-1}^{i}\right) f_{x}\left(x_{k} \mid x_{k-1}^{i}\right)}{f_{y, u}\left(y_{k}, u_{k} \mid x_{k-1}^{i}\right)} .
$$

As a result,

$$
\omega_{k}^{i}=\omega_{k-1}^{i} \int f_{y, u}\left(y_{k}, u_{k} \mid x_{k}\right) f_{x}\left(x_{k} \mid x_{k-1}^{i}\right) d x_{k} .
$$

To solve the above integral, the posterior is required, which is still unavailable. As a result, the choice of the importance density $g_{x}$ is a critical design decision for a PF. In the generic PF, the importance density is chosen equal to the prior, i.e.: $g_{x}\left(x_{k} \mid x_{k-1}^{i}, y_{k}, u_{k}\right)=f_{x}\left(x_{k} \mid x_{k-1}^{i}\right)$, which reduces Equation (6.10) to

$$
\omega_{k}^{i} \propto \omega_{k-1}^{i} f_{y, u}\left(y_{k}, u_{k} \mid x_{k}^{i}\right)
$$

At any step $k$, the particles $x_{k}^{i}$ are independent from the input of the same step $u_{k}$, i.e.: $f_{u}\left(u_{k} \mid x_{k}^{i}\right)=f_{u}\left(u_{k}\right)$. The weights can therefore be approximated as

$$
\omega_{k}^{i} \propto \omega_{k-1}^{i} f_{y}\left(y_{k} \mid x_{k}^{i}, u_{k}\right) .
$$

The propagated particles and their weights $\left(x_{k}^{i}, \omega_{k}^{i}\right)$ represent the posterior density at step $k$, and are used for drawing the priors of the next step. In practice, after a few iterations, a single particle takes the whole weight and the others die out. The phenomenon, called degeneracy, can be mitigated by constructing an empirical distribution function for the posterior, and resampling from the posterior distribution [152].

\subsubsection{RPF}

With a discrete density function, the particles with larger weights are likely to be repetitively drawn for the next steps. This can result in loss of diversity among the sampled particles and lead to sample impoverishment. To address this problem, Musso et 
al proposed regularize particle filter (RPF), where a continuous approximation of the posterior density is constructed for resampling [91]. An effective method for regularization of discrete density functions is using rescaled kernel density $K_{\mathrm{h}}(x)=$ $\mathrm{h}^{-D} K(x / \mathrm{h})$. Equation (6.9) can therefore be regularized as

$$
f_{x}\left(x_{k} \mid Y_{k}, U_{k}\right) \approx \sum_{i=1}^{m} \omega_{k}^{i} K_{\mathrm{f}}\left(x_{k}-x_{k}^{i}\right)
$$

where $D$ is the dimension of the state vector, and $h$ is a positive scalar for the bandwidth, which can be optimally found under the assumption of the Gaussian density and identity covariance matrix [174]. There are many choices for the kernel functions; two commonly used kernel functions are Epanechnikov and Gaussian functions. The steps of the RPF technique for state estimation for general NSIs is outlined in table 6.1.

Table 6.1. RPF-NSI technique

$$
\left\{\left(x_{k}^{i}, \omega_{k}^{i}\right)\right\}_{i=1: m}=\operatorname{RPF}\left[\left\{\left(x_{k-1}^{i}, \omega_{k-1}^{i}\right)\right\}_{i=1: m}, y_{k}, u_{k}\right]
$$

- Draw input samples:

- Propagate priors:

- Update the weights:

- Construct posterior density: $f_{x}\left(x_{k} \mid y_{k}, u_{k}\right) \approx \sum_{i=1}^{m} \omega_{k}^{i} K_{\mathrm{f}}\left(x_{k}-x_{k}^{i}\right)$

- Resample:

$$
\begin{aligned}
u_{k}^{i} \sim f_{u}\left(u_{k}\right) \\
x_{k}^{i} \sim f_{x}\left(x_{k} \mid x_{k-1}^{i}, u_{k}^{i}\right) \\
\omega_{k}^{i} \propto \omega_{k-1}^{i} f_{y}\left(y_{k} \mid x_{k}^{i}, u_{k}^{i}\right)
\end{aligned}
$$$$
\left\{\left(x_{k}^{i}, \omega_{k}^{i}=1 / m\right)\right\}_{i=1: m} \sim f_{x}\left(x_{k} \mid y_{k}, u_{k}\right)
$$

\subsubsection{APF}

In many occasions, the propagated particles land in the regions with low likelihoods. To improve the performance of state estimation in such cases, auxiliary particle filter (APF) draws the particles from the joint distribution of both the prior and the likelihood, i.e., the importance density is defined the joint density of the state and the index of the particle in the previous step $(k-1)$. As a result, the indices of those particles, which lead to higher likelihood of the measurement, are more prone to be selected. For a NSI, it leads to

$$
f_{x, j}\left(x_{k}, j \mid Y_{k}, U_{k}\right) \propto f_{y, u}\left(y_{k}, u_{k} \mid x_{k}\right) g_{x, j}\left(x_{k}, j \mid Y_{k-1}, U_{k-1}\right)
$$




$$
=\omega_{k-1}^{j} f_{y, u}\left(y_{k}, u_{k} \mid x_{k}\right) f_{x}\left(x_{k} \mid x_{k-1}^{j}\right) .
$$

The choice of the $j^{\text {th }}$ particle helps approximate the prior in Equation (6.12) by a representative value $\mu_{k}^{j}$ from the prior. The representative $\mu_{k}^{j}$ may be chosen the mean, mode or any random draw from the prior. As a result,

$$
f_{x, j}\left(x_{k}, j \mid Y_{k}, U_{k}\right) \approx \omega_{k-1}^{j} f_{y, u}\left(y_{k}, u_{k} \mid \mu_{k}^{j}\right) f_{x}\left(x_{k} \mid x_{k-1}^{j}\right) .
$$

Equation (6.10) is therefore reduced to

$$
\omega_{k}^{i} \propto \frac{f_{y, u}\left(y_{k}, u_{k} \mid x_{k}^{i}\right)}{f_{y, u}\left(y_{k}, u_{k} \mid \mu_{k}^{j^{i}}\right)} .
$$

Similar to Equation (6.14), the weights can be approximated by

$$
\omega_{k}^{i} \propto \frac{f_{y}\left(y_{k} \mid x_{k}^{i}, u_{k}\right)}{f_{y}\left(y_{k} \mid \mu_{k}^{j^{i}}, u_{k}\right)} .
$$

It should be noted that $j$ is the index of the drawn particles in the previous step, given the output and the input in the current step. It implies that, it is more likely to draw the particles, which will propagate to the states with higher output observation likelihood, given the system input. The density function for the candidate particles is therefore

$$
f_{x}\left(x_{k-1} \mid y_{k}, u_{k}\right) \approx \sum_{j=1}^{m} \omega_{k-1}^{j} f_{y}\left(y_{k} \mid \mu_{k}^{j}, u_{k}\right) \delta\left(x_{k-1}-x_{k-1}^{j}\right) .
$$

Table 6.2 presents the process of APF technique for NSI state estimation. 
Table 6.2. APF-NSI technique

$$
\left\{\left(x_{k}^{i}, \omega_{k}^{i}\right)\right\}_{i=1: m}=\operatorname{APF}\left[\left\{\left(x_{k-1}^{i}, \omega_{k-1}^{i}\right)\right\}_{i=1: m}, y_{k}, u_{k}\right]
$$

- Draw input samples:

$$
\begin{aligned}
& u_{k}^{i} \sim f_{u}\left(u_{k}\right) \\
& \text { - Generate representatives: } \quad \mu_{k}^{i}=E\left(x_{k} \mid x_{k-1}^{i}, u_{k}^{i}\right)
\end{aligned}
$$$$
\text { - Find representative weights: } \quad \omega_{\mu_{k}}^{i} \propto \omega_{k-1}^{i} f_{y}\left(y_{k} \mid \mu_{k}^{i}, u_{k}^{i}\right)
$$

- Construct representative density:

- Resample:

- Redraw input samples:

- Generate representatives:

- Draw priors:

- Update the weights:

- Construct posterior density: $f_{x}\left(x_{k} \mid y_{k}, u_{k}\right) \approx \sum_{i=1}^{m} \omega_{k}^{i} \delta\left(x_{k}-x_{k}^{i}\right)$

- Resample:

$$
x_{k-1}^{i} \sim f_{x}\left(x_{k-1} \mid y_{k}, u_{k}\right)
$$$$
u_{k}^{i} \sim f_{u}\left(u_{k}\right)
$$$$
\mu_{k}^{i}=E\left(x_{k} \mid x_{k-1}^{i}, u_{k}^{i}\right)
$$$$
x_{k}^{i} \sim f_{x}\left(x_{k} \mid x_{k-1}^{i}, u_{k}^{i}\right)
$$

$$
\left\{\left(x_{k}^{i}, \omega_{k}^{i}=1 / m\right)\right\}_{i=1: m} \sim f_{x}\left(x_{k} \mid y_{k}, u_{k}\right)
$$

$$
f_{x}\left(x_{k-1} \mid y_{k}, u_{k}\right) \approx \sum_{i=1}^{m} \omega_{\mu_{k}}^{i} \delta\left(x_{k-1}-x_{k-1}^{i}\right)
$$$$
\omega_{k}^{i} \propto f_{y}\left(y_{k} \mid x_{k}^{i}, u_{k}^{i}\right) \cdot / f_{y}\left(y_{k} \mid \mu_{k}^{i}, u_{k}^{i}\right)
$$

\subsubsection{Performance Evaluation on Benchmark Model}

In Chapter 4, PF was extended to state estimation of input-free systems with nonuniform steps and nonparametric noise structure, and the well-known univariate nonstationary growth model (UNGM) [155] was extended to verify the developed framework as a benchmark. For NSIs, state estimation will be more challenging, due to the more complexity and the higher uncertainty created by stochastic input. To examine the developed state estimation framework, we introduce a stochastic input to the extended benchmark model, and redefine the state and the output functions as below

$$
\begin{aligned}
& u_{k}=u_{A_{k}}+\kappa_{k} \\
& x_{k}=\frac{1}{2} x_{k-1} \Delta t_{k}+\frac{25\left(x_{k-1}+u_{A_{k}}\right) \Delta t_{k}}{1+\left(x_{k-1}+u_{A_{k}}\right)^{2} \Delta t_{k}{ }^{2}}+8 \Delta t_{k} u_{A_{k}} \cos (1.2(k-1)) /\left|u_{A_{k}}\right|+\tau_{k}(6 \\
& y_{k}=\frac{1}{20} x_{k}\left(x_{k}+u_{A_{k}}\right)+v_{k}
\end{aligned}
$$

The following assumptions are considered for the model: 
1- The time-steps $\Delta t_{k}=t_{k}-t_{k-1}$ have random length with an arbitrary distribution.

2- The input noise $\kappa_{k}$, the process noise $\tau_{k}$, and the measurement noise $v_{k}$ have unknown distributions, each one represented by s samples.

3- The input $u_{k}$, the state $x_{k}$, the output $y_{k}$ and their noises can take complex values.

4- The actual input value of the system is assumed:

$u_{A_{k}}=\sin (1.2 k)+\sin \left(k^{2}\right)$, for univariate system, and

$u_{A_{k}}=8(1+i)\left(\sin (1.2 k)-i \sin \left(k^{2}\right)\right)$, for complex system.

We apply the developed state estimation frameworks, i.e., RPF-NSI and APF-NSI on the extended benchmark model set forth in Equations (6.21) to (6.23). Two scenarios are taken into account for the model: i) the system is a UNGM with real values; ii) the system is a bivariate nonstationary growth model (BNGM) with complex values. Simulation results are presented as the following.

\subsubsection{UNGM}

We assume $\Delta t_{k} \sim \mathcal{U}(0,2)$ and $\tau_{k} \sim \mathcal{N}(0,4)$. The measurement noise and the input noise are represented by the sample sets $\left\{v_{k}^{l}\right\}_{l=1: 100}$ and $\left\{\kappa_{k}^{l}\right\}_{l=1: 100}$, drawn from a randomly weighted average of three distributions, i.e., normal, gamma, and Rayleigh, such that $E\left(v_{k}^{l}\right)=E\left(\kappa_{k}^{l}\right)=0$ and $\operatorname{Var}\left(v_{k}^{l}\right)=\operatorname{Var}\left(\kappa_{k}^{l}\right)=1$ for all time-steps. By sampling random noises from $\left\{v_{k}^{l}\right\}_{l=1: 100}$ and $\left\{\kappa_{k}^{l}\right\}_{l=1: 100}$ sets, Figure 6.1 shows the actual and the observed input, as well as the observed output of the system in 40 time-steps. Applying RPF and APF with 100 particles, Figure 6.2 presents the results in 10 iterations. We use the root mean square error as a metric for comparing the results. RPF shows an error of 5.41, whereas the error with APF is 6.52 that indicate the overall performance of RPFNSI is superior to APF-NSI in UNGM. 

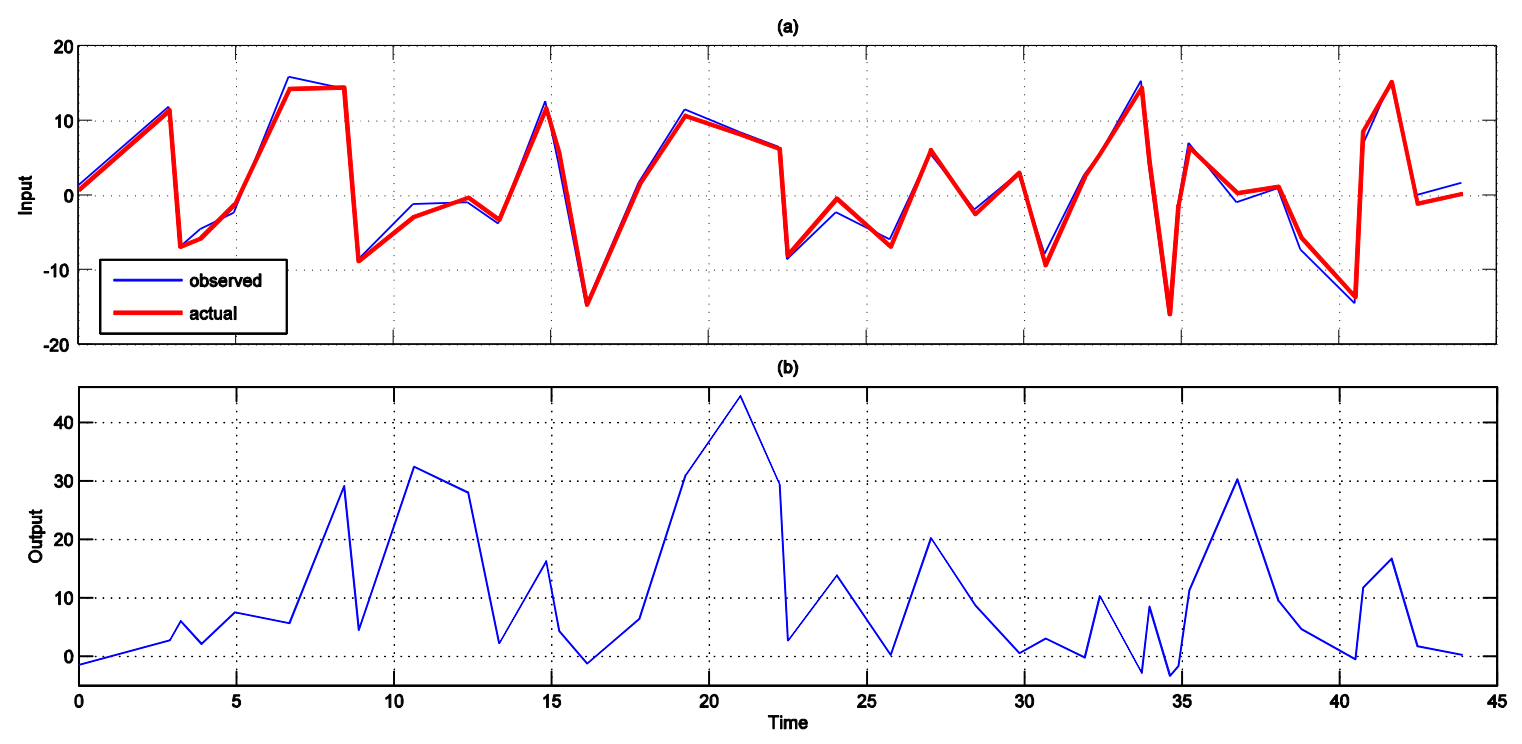

Figure 6.1. (a) System input, and (b) Observed output.
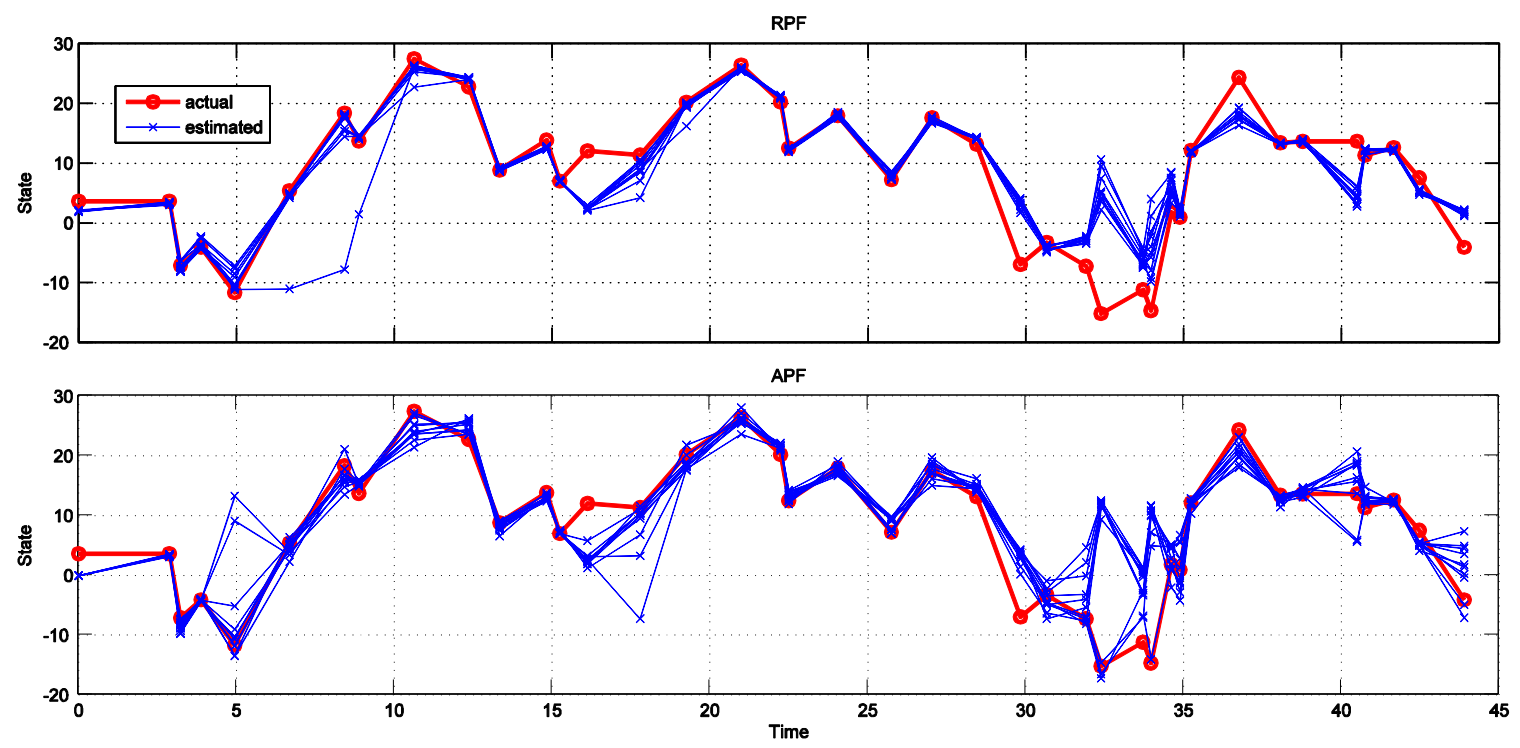

Figure 6.2. NSI state estimation by RPF and APF.

\subsubsection{BNGM}

The extended benchmark model adopts complex numbers for the input, the state and the output values. Therefore, we can verify the developed RPF-NSI and APF-NSI state estimators with a bivariate system model. For the simulation, we assume $\Delta t_{k} \sim \mathcal{U}(0,2)$ 
and $\tau_{k}=\tau_{k_{R}}+i \tau_{k_{I}}$, where $\tau_{k_{R}}, \tau_{k_{I}} \sim \mathcal{N}(0,4)$. The measurement noise and the input noise are represented by the sets $\left\{v_{k}^{l}\right\}_{l=1: 100}$ and $\left\{\kappa_{k}^{l}\right\}_{l=1: 100}$, where $v_{k}^{l}=v_{k_{R}}^{l}+i v_{k_{I}}^{l}$ and $\kappa_{k}^{l}=\kappa_{k_{R}}^{l}+i \kappa_{k_{I}}^{l}$. The real parts and the imaginary parts of the noses are drawn from a similar distribution as of UNGM, such that $E\left(v_{k}^{l}\right)=E\left(\kappa_{k}^{l}\right)=0$ and $\operatorname{Var}\left(v_{k_{R}}^{l}\right)=$ $\operatorname{Var}\left(v_{k_{I}}^{l}\right)=\operatorname{Var}\left(\kappa_{k_{R}}^{l}\right)=\operatorname{Var}\left(\kappa_{k_{I}}^{l}\right)=1$ at each time-step $k$. To run the simulation, we sample random noses from the representative sets. In Figure 3, the bivariate input and output of the system are shown. Figure 6.4 shows the actual and the estimated state components in 10 trials using RPF and APF with 100 particles. Similar to UNGM, RPF shows a better performance; the root mean square error with RPF is 3.89 and 4.83 for the real part and the imaginary part respectively, whereas, these errors are 5.34 and 7.13 for APF. Repeated simulations with UNGM and BNGM presented similar results, suggesting RPF a better state estimation tool for NSIs in the settings set forth. Table 3 provides the modeling errors averaged over 100 simulations.
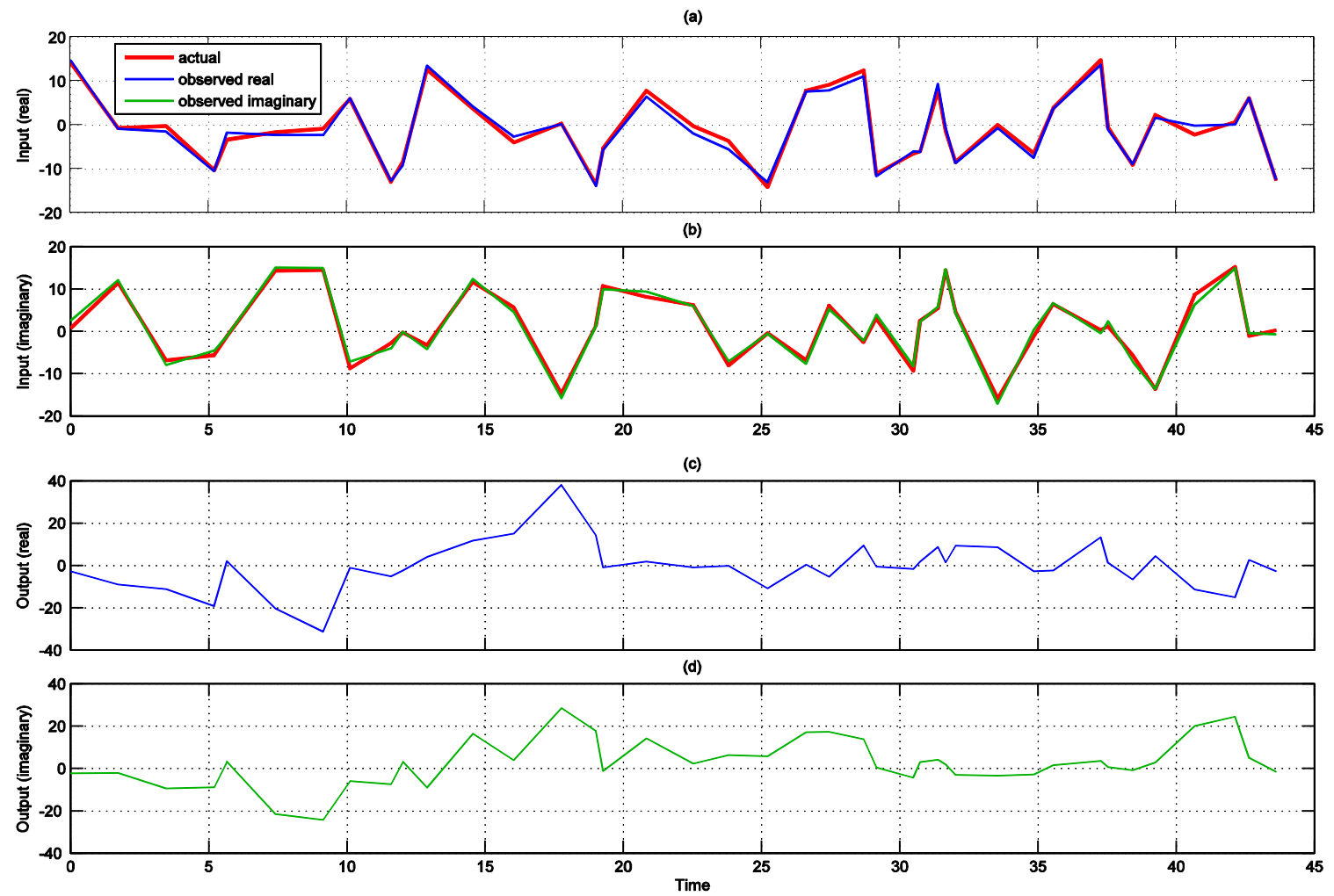

Figure 6.3. System input and output: (a) Input real part, (b) Input imaginary part, (c) Observed output real part, and (d) Observed output imaginary part. 

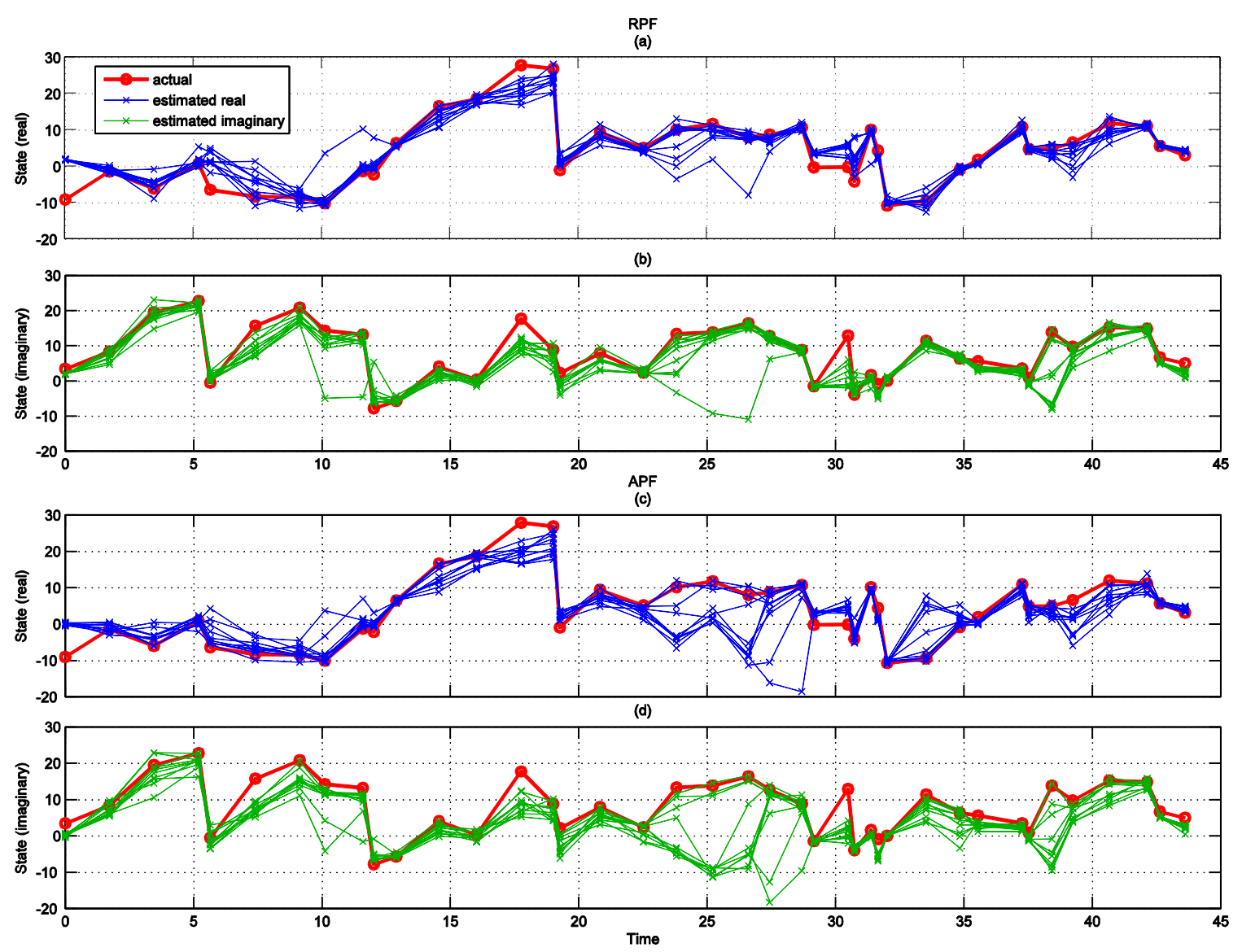

Figure 6.4. Bivariate NSI state estimation (a) RPF real part, (b) RPF imaginary part, (c) APF real part, and (d) APF imaginary part.

Table 6.3. State estimation errors averaged over 100 simulations

\begin{tabular}{|c|c|c|c|}
\hline \hline \multirow{2}{*}{ Technique } & \multirow{2}{*}{ UNGM } & \multicolumn{2}{|c|}{ BNGM } \\
\cline { 3 - 4 } & & Re. part & Im. part \\
\hline \hline RPF-NSI & 5.41 & 3.89 & 4.83 \\
\hline APF-NSI & 6.52 & 5.34 & 7.13 \\
\hline
\end{tabular}

\subsection{Turbine Degradation Modeling}

In most of the GTEs, a number of measurements from the operating parameters of the machine are regularly logged through the monitoring system. Such data are logged 
sequentially, and if appropriately analyzed, they can provide valuable information about the health status of the GTE components. In the previous works, the authors developed a comprehensive thermodynamic model for single shaft GTEs to analyze the relation between the GTE measurements and predict the gas-path parameters (GPP) $[25,175]$. The approach is utilized here to predict the degradation level of the turbine.

\subsubsection{Gas-path Model of the Degrading Turbine}

GTE operating systems commonly measure and monitor the power $(P W)$, the rotational speed $(N)$ and the exhaust gas temperature $(E G T)$. Once the GTE model is constructed and calibrated with the design parameters $(D P), G P P$ can be predicted by the model, given the ambient condition and two of the three mentioned operating parameters [97]. Therefore, for a demanded power $P W$ and controlled rotational speed $N$,

$$
[G P P]=\mathrm{F}_{G T E}\left(D P, T_{C i}, P_{C i}, \phi_{C i}, N, P W\right),
$$

where, $T_{C i}, P_{C i}$ and $\phi_{C i}$ are the total temperature, the total pressure and the relative humidity of the intake air respectively. During the degradation, the actual design parameters of the GTE gradually deviate from the brand-new condition, and affects the GTE output as a result.

In the turbine section of the GTE, the performance map provides the corrected mass flow $W_{T_{c o r}}$ and the isentropic efficiency $\eta_{T}$, given the corrected rotational speed $N_{T_{\text {cor }}}$, and the pressure ratio $P R_{T}$,

$$
\left[N_{T_{c o r}} / N_{D} \times W_{T_{c o r}}, \eta_{T}\right]=\operatorname{Map}_{T}\left(N_{T_{c o r}}, P R_{T}\right)
$$

where $N_{D}$ is the rotational speed at the design point. The mass flow of the turbine $W_{T}$ is then found from the corrected mass flow [175]. By degradation of the turbine components and deviation of the design parameters, the isentropic efficiency of the turbine section decreases and the mass flow increases under the same operating conditions. We define the turbine degradation vector as $\left[\rho_{\eta}, \rho_{W}\right]$, where $\rho_{\eta}$ is the fraction of isentropic efficiency decrease, and $\rho_{W}$ is the fraction of mass flow capacity increase 


$$
\begin{aligned}
& \rho_{\eta}=\left(\eta_{T}-\eta_{T_{A}}\right) / \eta_{T}, \\
& \rho_{W}=\left(W_{T_{A}}-W_{T}\right) / W_{T},
\end{aligned}
$$

where $\eta_{T_{A}}$ and $W_{T_{A}}$ are the actual efficiency and mass flow of the turbine respectively. When the degradation of the turbine is the objective, all design parameters remain unchanged except those in the turbine section, which are represented by the degradation vector $\left[\rho_{\eta}, \rho_{W}\right]$, replacing $D P$ in Equation (6.24). Given the ambient conditions and the operating parameters, GPP depend on $\left[\rho_{\eta}, \rho_{W}\right]$ variations. Alternatively, if two elements of GPP become available, the two degradation symptoms, i.e.: $\rho_{\eta}$ and $\rho_{W}$ can be determined by Equation (6.24). Figure 6.5 shows examples of the predicted GPP versus $\rho_{\eta}$ and $\rho_{W}$, using Equation (6.24) for a typical GTE.
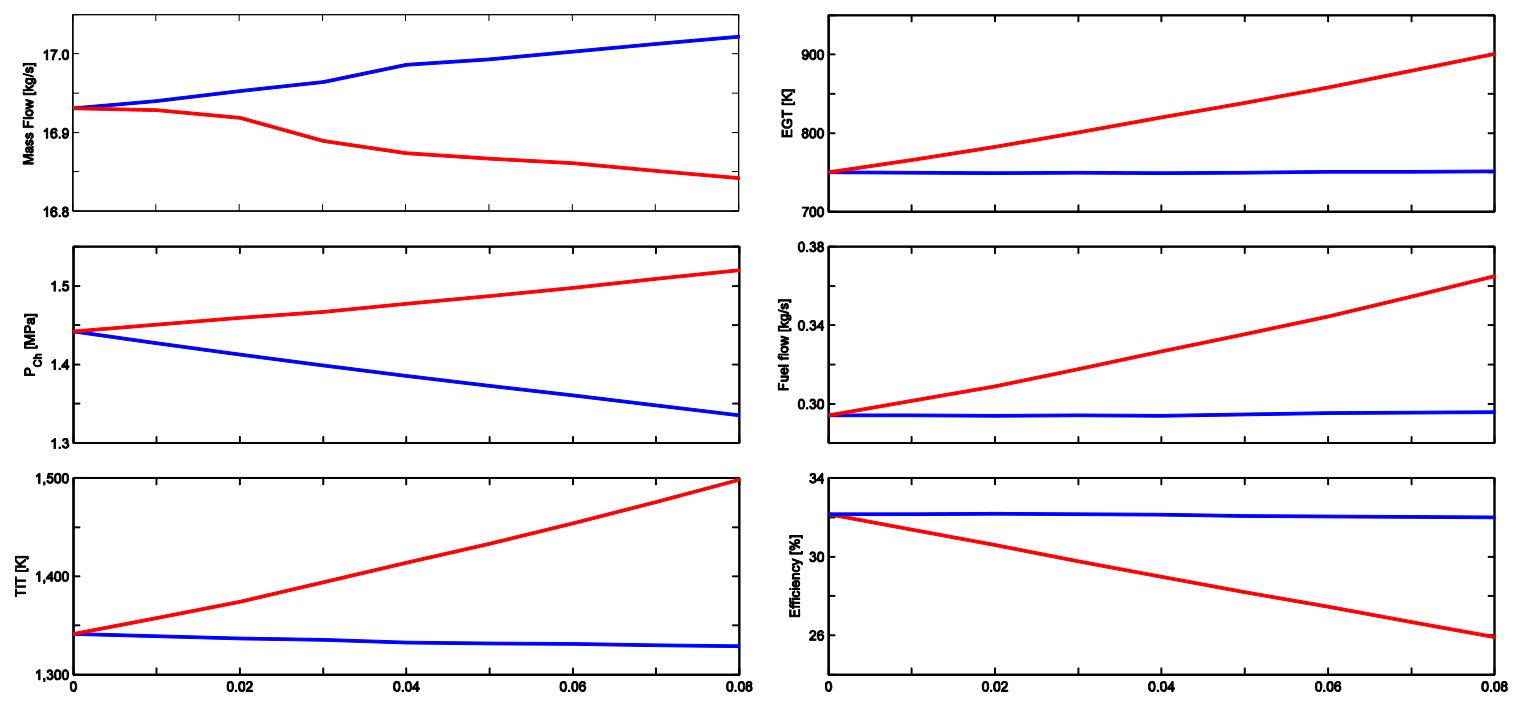

Figure 6.5. Variation of typical $G P P$ vs efficiency decrease $\rho_{\eta}$ and mass flow increase $\rho_{W}$. Operating parameters are fixed at $T_{C i}=15^{\circ} \mathrm{C}, P_{C i}=101 \mathrm{kPa}, \phi_{C i}=50 \%, N=16500 \mathrm{RPM}, P W=4 \mathrm{MW}$.

EGT is always monitored by the GTE operating systems, however, the other parameters are not measured in many GTEs including the one in this research. Instead, some external parameters such as fuel pressure $\left(P_{F}\right)$ and fuel valve angle $(V A)$ are accessible with no additional instrumentation. Pressure of the combustion chamber $\left(P_{C h}\right)$ 
affects the pressure of the injected fuel. It can be consequently estimated as a function of the fuel pressure, the fuel flow $\left(W_{F}\right)$ and the valve factor represented by $V A$.

$$
P_{C h}=\mathrm{F}_{\text {valve }}\left(P_{F}, W_{F}, V A\right) .
$$

As shown in Figure 6.5, $P_{C h}$ and $W_{F}$ are found from Equation (6.24) and add no more unknowns to the equations. As a result, combination of Equations (6.24) and (6.28) reduces to

$$
\left[E G T, P_{F}\right]=\mathrm{F}_{G T E / v a l v e}\left(\left[\rho_{\eta}, \rho_{W}\right],\left[T_{C i}, P_{C i}, \phi_{C i}, N, P W, V A\right]\right) .
$$

Equation (6.29) provides a model-based relation between the system state $\left[\rho_{\eta}, \rho_{W}\right]$, the input $\left[T_{C i}, P_{C i}, \phi_{C i}, N, P W, V A\right]$, and the output $\left[E G T, P_{F}\right]$. Although within the small variations of the state, the outputs exhibit a quasi-linear dependency to the state, it should be noted that the relation between system input and output is nonlinear in the GTEs.

To assess the effectiveness of the developed model, measurements from three-year operation of a single shaft industrial GTE are utilized. The data have been logged once every two hours during an entire period between consecutive overhauls. At each reading, a number of GTE measurements have been logged including the above-mentioned input and output of the system. Figure 6.6 shows selected GTE measurements during the operating period. It should be noted that there are some recoverable performance deterioration mechanisms, such as compressor fouling that create short-term effects in the performance of the GTE. The short-term effects can be removed by washing and cleaning the GTE components. In order to eliminate such effects from the measurements, we collect the data from a five-day time window after each compressor wash. The choice of the window size is the result of a trade-off between gaining more statistical information by enlarging the sample size, and decreasing the fouling effects on the data by shortening the sampling duration [97]. Using the GTE measurements after the compressor washes in Equation (6.29), we calculate the degradation symptoms $\left[\rho_{\eta}, \rho_{W}\right]$, attributed to the turbine degradation only. Scattered data points in Figure 6.7 correspond to the calculated $\rho_{\eta}$ and $\rho_{W}$ within 60 measurements steps after each wash. Throughout the operating period, an overall increase is observable for both of the degradation symptoms. Local fluctuations of the symptoms are attributed only to the measurement noise of the input 
and the output, because the five-day sampling duration is too short to affect the long-term performance of the turbine. Measurement noise is uncharacterized in this setting; however, the distribution of the degradation symptoms can be estimated by the empirical joint probability distribution function (pdf) of $\left[\rho_{\eta}, \rho_{W}\right]$. Figure 6.8 shows the joint distribution of the symptoms, induced by the measurement noise, in three selected sampling time-steps.
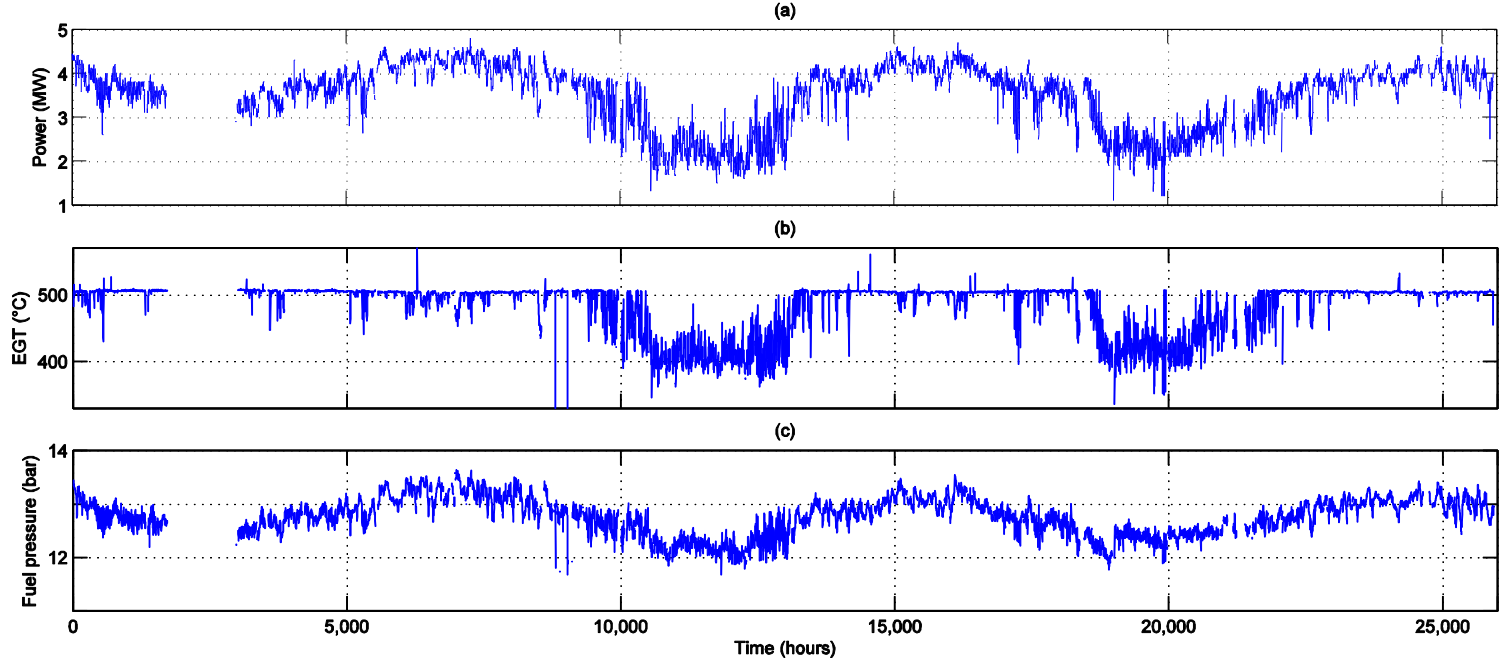

Figure 6.6. Measured operating parameters of the GTE: (a) Power, (b) EGT, and (c) fuel pressure.
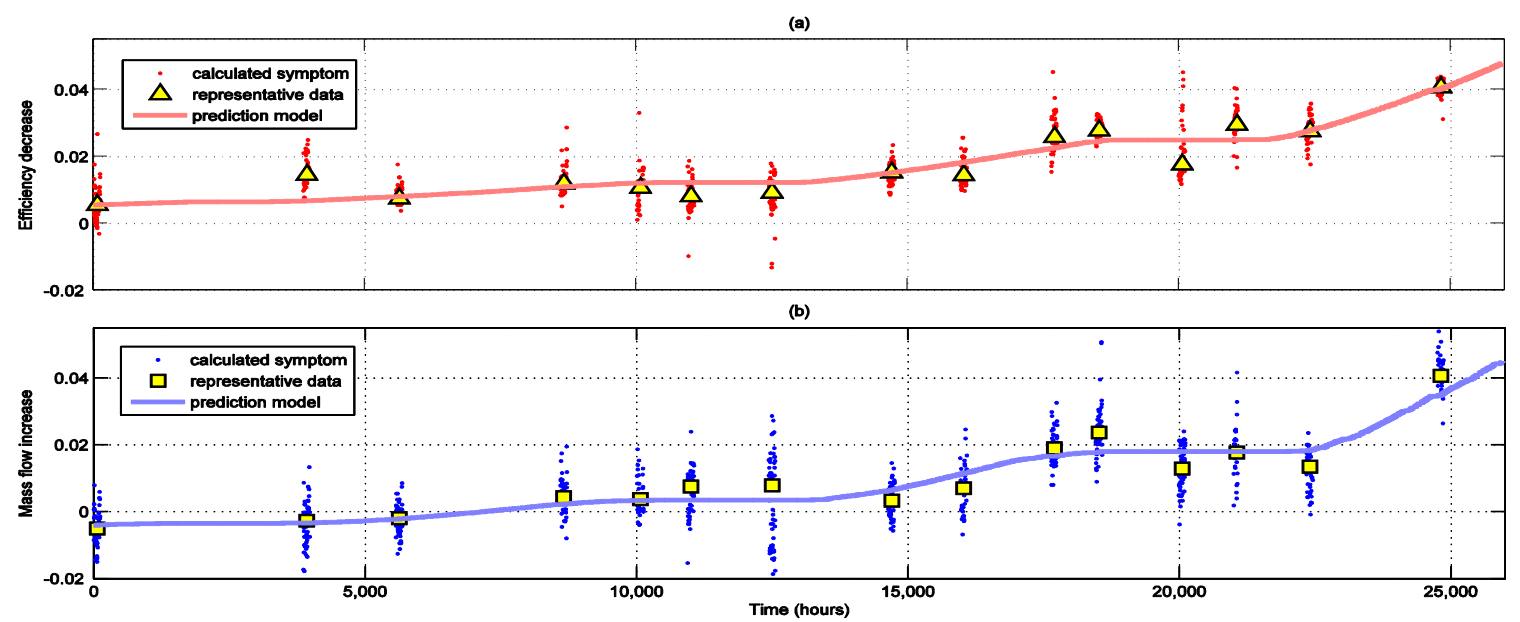

Figure 6.7. Variation of the turbine degradation symptoms during the operation, and corresponding prediction models: (a) $\rho_{\eta}$ symptom, and (b) $\rho_{W}$ symptom. 
The joint distribution of the degradation symptoms at each sampling time-step shows a large dependency between the sampled data, which is also non-Gaussian.

(a)

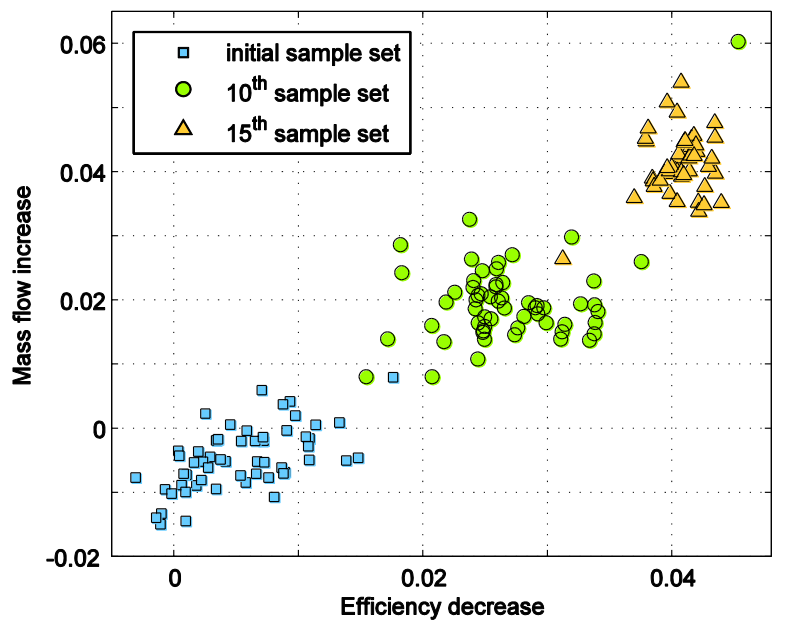

(b)

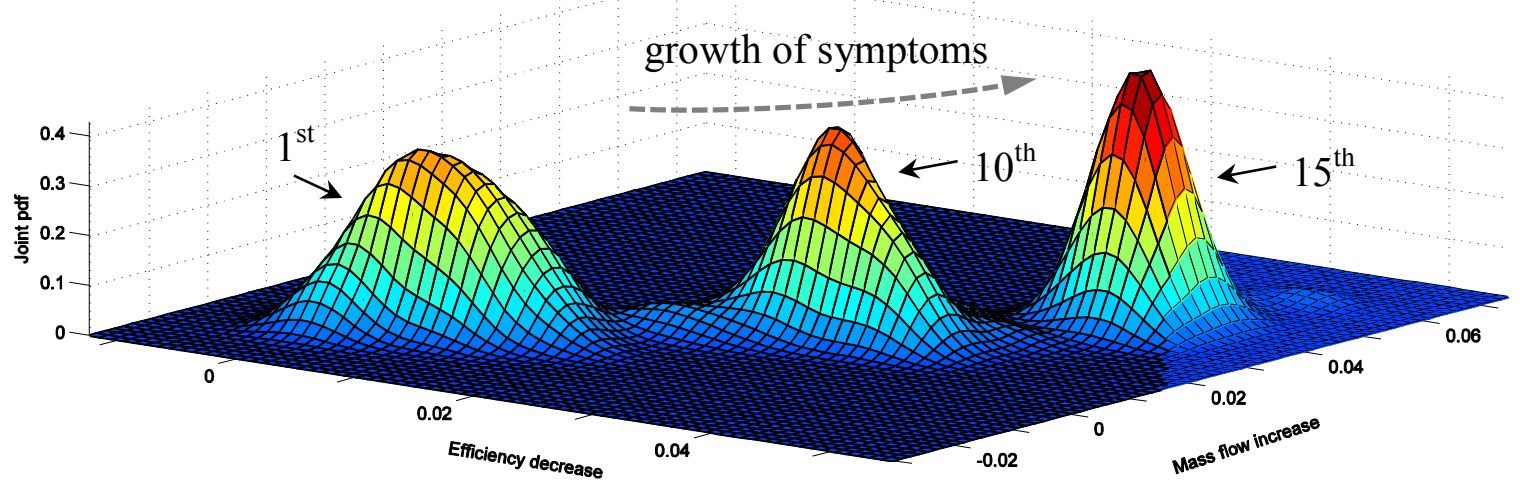

Figure 6.8. Degradation symptoms, sampled at time-step 1, 10 and 15; (a) distribution of $\left[\rho_{\eta}, \rho_{W}\right]$ in each sampling time-step, (b) Joint pdf of $\left[\rho_{\eta}, \rho_{W}\right]$ at each sampling time-step.

\subsubsection{Turbine Degradation Prediction Model}

Several research works have investigated the relation between fault modes and consequent degradation symptoms of the turbines [168-171], whereas fewer works have 
studied the growth of the degradation symptoms over time [1]. Beside the effect of time, it is expected to experience faster degradation under higher levels of the GTE power, which lead to harsher operating condition for the components. To examine the trend of the degradation symptoms, we assume the median of the data in each sample set as the representative of the central tendency of the corresponding set [150]. Figure 6.7 shows a periodic fluctuation in the growth rate of representative $\rho_{\eta}$ and $\rho_{W}$, e.g., from hour 8600 to 12500 , the symptoms do not change much, whereas from hour 12500 to 18500 , they tangibly increase. A comparison with Figure 6.6(a) suggests the degradation rates of the symptoms are correlated with the GTE power level, i.e., the symptoms grow faster when the power is high. This is basically attributed to the increase in firing temperature required for higher power. As a result, a candidate state evolution model should take into account the following considerations:

i. Both the time and the operating power contribute to the growth of the symptoms.

ii. Since the symptoms are caused by non-recoverable faults, the potential model should be monotonically increasing.

iii. The degradation nearly plateaus when the power falls below some partial load levels. iv. The overall growth rate of the symptoms increases over time.

To address the stated considerations, we introduce the following model:

$$
d \rho_{\eta} / d t=\psi e^{\lambda t}
$$

Where $\lambda$ is a constant coefficient and $\psi$ is a logistic function of the power with constant parameters $L, \alpha$ and $P W_{0}$.

$$
\psi=L /\left(1+e^{-\alpha\left(P W(t)-P W_{0}\right)}\right) .
$$

Function $\psi$ maintains the consideration iii and the exponential part satisfies the condition iv. Integrating Equation (6.30) over the discrete steps with $d t$ duration results in

$$
\rho_{\eta_{l_{k}}}=\rho_{0}+\sum_{l=1}^{l_{k}}\left[L e^{\lambda d t l} d t /\left(1+e^{-\alpha\left(P W_{l}-P W_{0}\right)}\right)\right] .
$$

There will be a similar model for $\rho_{W}$ prediction with corresponding parameters. The parameters can be found by best-fitting the model on the representative data, i.e., median of the bins. Table 6.4 gives the optimum model parameters for the two symptoms, and the model prediction results are shown as curves in Figure 6.7 At each sampling time-step $k$, 
there is an error due to the mismatch of the model prediction and the representative data $\rho_{\eta_{k}}^{*}$.

$$
\varepsilon_{\eta_{k}}=\rho_{\eta_{l_{k}}}-\rho_{\eta_{k}}^{*}
$$

Using Equation (6.32) for stepwise prediction results in modeling error $\tau_{k}=\left[\tau_{\eta_{k}}, \tau_{W_{k}}\right]$,

$$
\rho_{\eta_{k}}=\rho_{\eta_{k-1}}+\sum_{l=l_{k-1}+1}^{l_{k}}\left[L e^{\lambda d t l} d t /\left(1+e^{-\alpha\left(P W_{l}-P W_{0}\right)}\right)\right]+\tau_{\eta_{k}}
$$

Therefore,

$$
\tau_{\eta_{k}}=\varepsilon_{\eta_{k}}-\varepsilon_{\eta_{k-1}}
$$

and similarly,

$$
\tau_{W_{k}}=\varepsilon_{W_{k}}-\varepsilon_{W_{k-1}}
$$

As a result, modeling uncertainty can be represented by the empirical joint distribution of the errors over $n$ samples,

$$
f_{\tau}(\tau) \approx \sum_{k=1}^{n} K_{\mathrm{h}}\left(\tau-\tau_{k}\right)
$$

The resulting joint pdf is shown in Figure 6.9.

Table 6.4. Prediction model parameters for $\rho_{\eta}$ and $\rho_{W}$

\begin{tabular}{|c|c|c|c|c|c|}
\cline { 2 - 6 } \multicolumn{1}{c|}{} & $L$ & $\alpha$ & $P W_{0}$ & $\rho_{0}$ & $\lambda$ \\
\hline \hline$\rho_{\eta}$ & $4.8852 \mathrm{E}-7$ & 0.0504 & 3231 & $0.5573 \mathrm{E}-2$ & $1.0532 \mathrm{E}-4$ \\
\hline$\rho_{W}$ & $7.8320 \mathrm{E}-7$ & 0.0500 & 3709 & $-0.4089 \mathrm{E}-2$ & $1.0527 \mathrm{E}-4$ \\
\hline
\end{tabular}




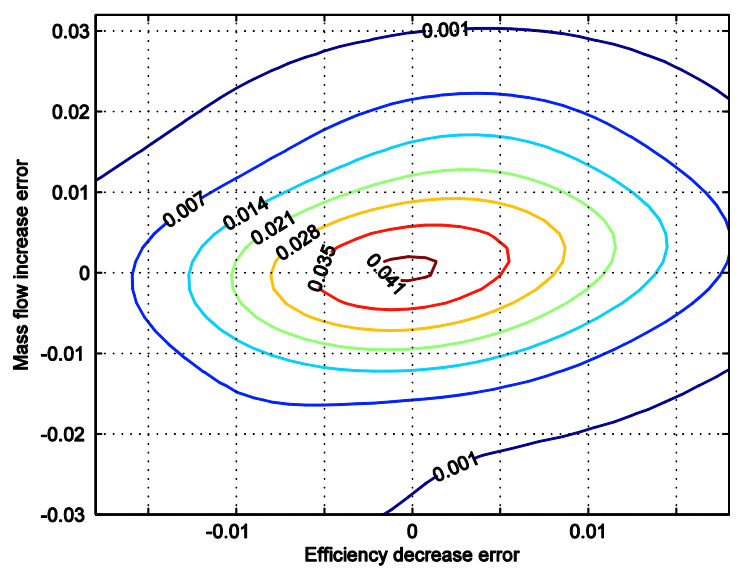

Figure 6.9. Empirical joint distribution of the modeling error.

\subsubsection{Degradation state estimation}

Based on the results for the measurement model in 6.3.1, and the state evolution model developed in 6.3.2, one should consider the following conditions for state estimation problem of turbine degradation symptoms:

- Both the measurement model and the state evolution model are nonlinear.

- Measurement noise and state evolution modeling uncertainty are represented by sample measurements/errors. The corresponding distributions are correlated and nonGaussian in either case.

The conditions imply that classic state estimation techniques such as Kalman filter (KF) shall not be expected to yield accurate results. The developed RPF-NSI framework in section 6.2 showed reliable performance for such systems, and therefore, we obtain that framework for turbine degradation state estimation in this section.

We notice that the measurement noise is unknown for both the input $\left[T_{C i}, P_{C i}, \phi_{C i}, N, P W, V A\right]$ and the output $\left[E G T, P_{F}\right]$. Redundant measurements are utilized instead to characterize the likelihood in Equation (6.13), where

$$
f_{y, u}\left(y_{k}, u_{k} \mid x_{k}^{i}\right)=f_{x}\left(x_{k}^{i} \mid y_{k}, u_{k}\right) f_{y, u}\left(y_{k}, u_{k}\right) / f_{x}\left(x_{k}^{i}\right)
$$

and $f_{y, u}\left(y_{k}, u_{k}\right)=\int f_{y, u}\left(y_{k}, u_{k} \mid x_{k}^{i}\right) f_{x}\left(x_{k}^{i}\right) d x_{k}^{i}$ is independent from the choice of particle $x_{k}^{i}$. At the same time, the particles are resampled from the prior density and they are equally likely. Consequently, 


$$
\begin{aligned}
\omega_{k}^{i} & \propto \omega_{k-1}^{i} f_{x}\left(x_{k}^{i} \mid y_{k}, u_{k}\right) \\
& \propto \omega_{k-1}^{i} \sum_{j=1}^{s} K_{\mathrm{h}}\left(x_{k}^{i}-x_{k}^{j}\right) .
\end{aligned}
$$

where $x_{k}^{j}$ satisfies the deterministic part of the measurement model for $s$ redundant measurements $(j=1: s)$,

$$
y_{k}^{j}=G\left(x_{k}^{j}, u_{k}^{j}\right)
$$

Equations (6.34) and (6.37) constitute the state evolution model, and Equation (6.39) updates the particle weights, where $x_{k}^{i}=\left[\rho_{\eta_{k}}^{i}, \rho_{W_{k}}^{i}\right]$ are the particles.

Figure 6.10 shows 20 prediction trajectories by RPF (green lines) and the expected estimations over 50 runs for both RPF and APF techniques using 100 particles. There is no information available about the actual degradation of the turbine in the study, and the accuracy of the employed techniques cannot be evaluated from this prospect. The repeatability of the results is also comparable, i.e., the standard deviation of the results for both techniques is $\sim 2 \times 10^{-3}$ for $\rho_{\eta}$ and $\rho_{\mathrm{W}}$.
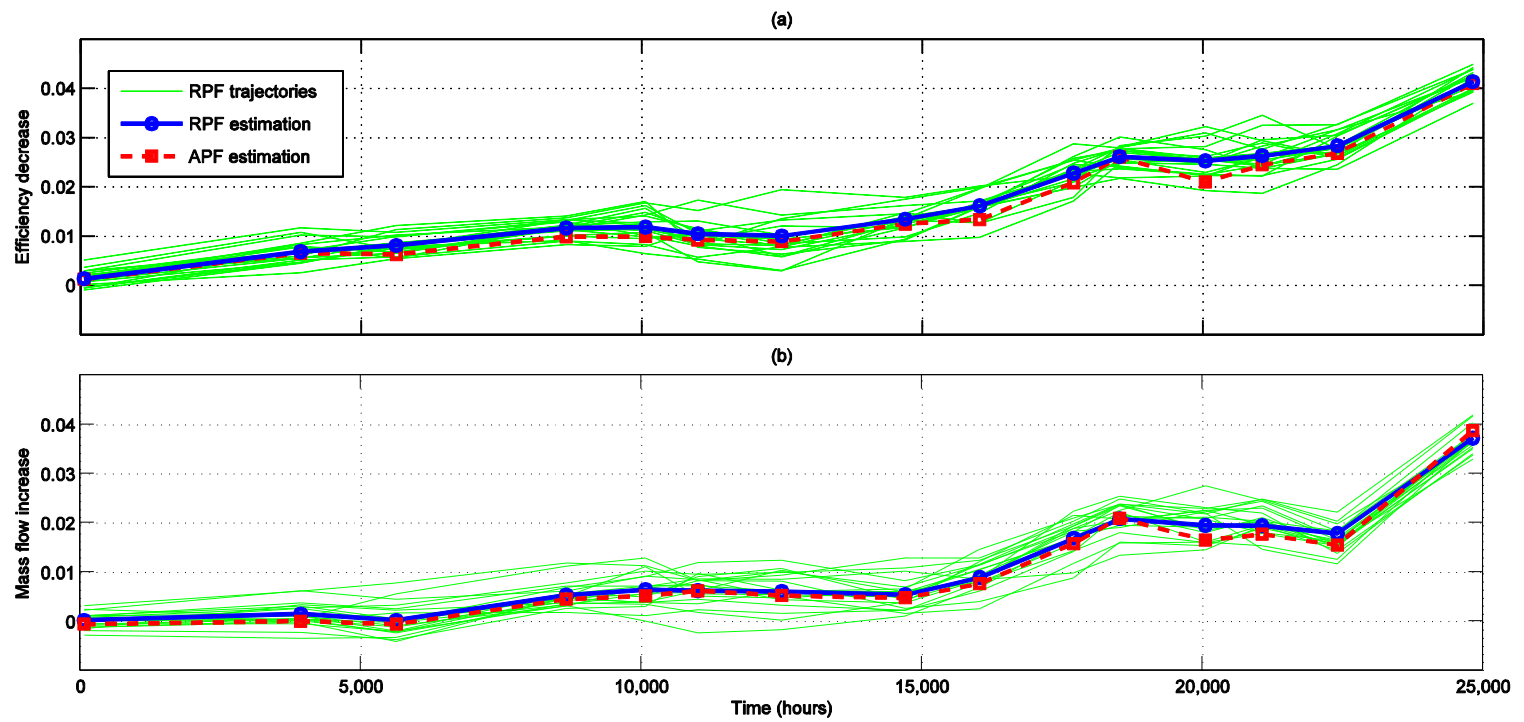

Figure 6.10. State estimation results by RPF (blue) and APF (red) using 100 particles: (a) $\rho_{\eta}$ symptom, and (b) $\rho_{W}$ symptom.

To compare the performance of the techniques in this setting, we take into account the 
non-recoverable nature of the turbine degradation phenomena. At sampling time-step 12, APF estimation shows a decline in both symptoms, and at sampling time-step 14 it shows another decline for $\rho_{W}$ symptom. With RPF, we observe much smaller declines in the mentioned sampling time-steps. As a result, the estimation of RPF shows a better agreement with the expert knowledge. The reason APF results in such fluctuations can be explained by the distribution of the calculated symptoms, based on the redundant measurements. As we see in Figure 6.7., the tendency of the calculated symptoms moves downward at sampling time-steps 12 for both of the symptoms, and at sampling time-step 14 for $\rho_{\mathrm{W}}$ symptom. RPF samples the particles with larger measurement likelihood in the upcoming steps. Therefore, the selected particles are more likely to land in the states, where the measurements dictate. Consequently, the resulting estimation is more influenced by the fluctuation of the measurements, as we see in Figure 6.10. for APF. In this setting, the estimation results by RPF acceptably comply with the reported literature $[142,156]$ and show a promising performance with the turbine degradation under stochastic input.

\subsection{Summary}

In this chapter, the PF scheme was extended to nonlinear/non-Gaussian dynamical systems, receiving noisy inputs. The measurements on the system and the state evolution model have non-parametrized noise structures, and the lengths of the time-steps are nonuniform. To examine the performance of the developed framework on multivariate systems, the well-known UNGM was extended to complex space as a BNGM with nonuniform time-steps. The simulation results showed a superior performance when RPF scheme was employed, compared to the performance of APF.

Three-year records of GTE operating data were utilized to examine the performance of the developed framework in the next step. A thermodynamic model of the gas-path and an empirical model for the fuel valve were developed to establish the measurement model of the GTE, in which the measurable input / output were attributed to the turbine degradation symptoms as the state of the system. To predict the evolved state in the next time-step, a novel prediction model was introduced for time-based performance deterioration of the turbine, considering the power level. Based on the background 
knowledge on the long-term degradation of the turbine, it was concluded that the performance of the developed framework is superior with RPF compared to APF. 


\section{Chapter 7: Contributions, Conclusions and Recommendations}

This chapter summarizes the contributions and conclusions of the preceding chapters to show how they coherently address the stated objectives of the work. Recommendations for future research work are provided subsequently.

\subsection{Contributions and Impacts}

In this research, four novel unified performance indicators based on heat balance model of single shaft GTEs were developed for performance monitoring. Proposed indicators can monitor overall GTE performance deterioration, including short-term and long-term deterioration trends. Using only the limited measurements from a GTE operating system, the developed indicators provide valuable information on health state of the GTE at system level and help the users optimize maintenance service scheduling.

Performance monitoring of GTE was improved by inclusion of humidity effects in GTE thermodynamic model. Local noise of the performance indicators was reduced and the corresponding prediction model showed an improved performance.

Sequential Monte Carlo state estimation method was extended to nonlinear/nonGaussian systems with statistically unknown noise structure and non-uniform time steps. The contribution enables the researchers to apply Particle Filter framework for state estimation of autonomous dynamical systems, where the noise characteristic of the measurement system is unknown.

Effect of humidity condensation on the trend of GTE compressor fouling was identified and a prediction model based on ANFIS structure was developed for GTE recoverable performance prediction. The results enable GTE operating companies to predict future fouling condition of the compressors and plan for maintenance services, using the GTE operating past data.

Particle Filters did not have capability to manage noisy inputs received by a dynamical system. In this work, sequential Monte Carlo method was developed for state estimation in the general class of non-autonomous dynamical systems with noisy inputs and outputs. The developed framework can be applied for state estimation of dynamical systems in 
various disciplines.

A bivariate non-stationary growth model was proposed to assess state estimation techniques with two-dimensional state and measurement vectors. The proposed model can be employed as a benchmark to verify effectiveness of any state estimation technique developed for dynamical systems.

A novel logistic-exponential growth model was proposed for turbine degradation symptoms, i.e., efficiency decrease and mass flow increase. The proposed model was verified on three-year operating data of a GTE and the results were in good agreement with the expert knowledge of the field. This model can be much helpful for the users, to predict long-term degradation in GTE hot sections.

\subsection{Summary and Conclusions}

In Chapter 2, a physics-based model for single shaft GTE was developed to take into account the operating conditions and calculate the gas path parameters using the limited measurements from the GTE operating system. The model was calibrated with the parameters of a brand-new GTE, and then it was utilized to calculate the expected baseline values for the EGT and the power of the GTE. Consequently, based on the deviation of the measurements from the respective baseline values, two performance indicators were proposed for monitoring the GTE performance. The indicators have explicit physical meanings, i.e., the heat loss index gives a measure of the extra heat wasted due to a hotter exhaust gas, and the power deficit index provides a scale for the lost power due to the GTE performance deterioration.

Investigation with experimental data from an industrial GTE showed that the developed indicators clearly revealed both the short-term and the long-term regimes of the GTE performance deterioration, caused by recoverable and non-recoverable degradations. The advantage with the developed indicators is that they are independent from the operating points and the ambient conditions. At the same time, they only utilize the limited operating data measured from the GTE operating system and need no additional sensors for data acquisition.

In Chapter 3, an enhanced thermodynamic model was developed to simulate the operating conditions of a single shaft GTE, considering the effects of humidity in the 
intake air. Another thermodynamic model was developed to find the moisture content of the GTE intake air added by the inlet air cooler. A new physics-based performance indicator, i.e. excess heat ratio was introduced for GTE performance monitoring, using the measurements from the operating system. The indicator was defined as the normalized difference between the actual enthalpy and the expected enthalpy of the exhaust gas, predicted for the brand-new GTE condition. A comparative study was performed on the performance of the indicator in two conditions; at first, the indicator was developed based on the primary model of the GTE, and then it was recalculated utilizing the enhanced GTE model. Numerical investigation using three-year operating records of an industrial GTE showed the following results: 1) the difference between the resulting indicators from two models showed a clear correlation with the moisture content in the ambient air. It indicates that inclusion of the humidity effects in GTE modeling provides more accurate results for the performance indicator, and 2) an exponential function was used to fit prediction models on the indicator signal. The comparison of the results suggested a better predictability for the case where the enhanced GTE model was employed.

A system identification strategy was set forth in Chapter 4 to quantify a degradation symptom of the turbine, i.e., the percentage of isentropic efficiency loss. In order to eliminate the effects of the compressor fouling, the data were collected from the first operating days after each compressor wash. The mentioned isentropic efficiency loss of the turbine was found at each step such that the GTE thermodynamic model could match the observed long-term performance deterioration of the GTE. To optimally estimate the variations of the isentropic efficiency loss of the turbine, a data-model fusion framework was developed based on RAPF technique with regards to the following conditions: 1) GTE compressors are usually washed in non-even periods, 2) the GTE gas-path model and the turbine degradation phenomenon are both nonlinear, and 3) the measurement signals have statistically unknown noise structure. Effectiveness of the developed framework was examined first on a benchmark univariate nonstationary growth model with known internal states, and then on the operating data of a GTE. In both cases, the developed framework showed a superior performance in accuracy and repeatability, compared to another framework, which was constructed using RPF technique. 
The developed framework extended the application of RAPF technique to nonuniform time steps and nonparametric noise structure. It showed promising results when applied for degradation state estimation in hot section of the GTE, using only the limited measurements from the GTE operating system.

In Chapter 5, short-term performance deterioration in the GTE was studied with respect to the corresponding effective factors in the ambient and the operating conditions. Performance deterioration in the GTE was quantified using two physics-based performance indicators developed in Chapters 3 and 4, and the corresponding signals were filtered with a PF-based data-model fusion filter for noise removal. It was known from the literature that three effective factors in the short-term performance deterioration are the GTE power, the intake mass flow and the rate of humidity condensation at the inlet of the compressor. Power is always available directly from the GTE operating system, and the intake mass flow can be predicted by the physics-based GTE model. To calculate the rate of humidity condensation, GTE operating data and the dimensions of the compressor inlet duct were utilized in a heat balance model. To establish a functional relation between the effective factors and the performance indicators, different combinations of the inputs and outputs were used to train the ANFIS model. The trained models for both performance indicators were then run under controlled operating scenarios. The resulting simulations clearly revealed the GTE deterioration behavior was influenced by the mentioned ambient and the operating conditions. Compared to $E R$ index, $E H$ showed a better performance in that, the resulting simulation profiles could clearly be explained by physics of the fouling phenomena. The rate of humidity condensation showed a complex effect in the short-term performance deterioration, i.e., depending on the magnitude of the humidity condensation rate, it sometimes deteriorated and sometimes improved the trend of performance variation. The slowest performance deterioration was observed with the dry air. With the increment of the humidity condensation rate, performance deterioration accelerated in the beginning and slowed down eventually. The highest rate for the performance deterioration was observed when the humidity condensation rate reached to $\sim 0.9 \%$ of the intake mass flow. After this level, the increase of the humidity condensation rate led to slower performance deterioration and an inverse regime that could be described by self-cleaning phenomenon. 
The developed technique was able to predict the short-term performance variations using the ambient and the operating records. It could also find the optimum humidity condensation rate, which can be maintained by the inlet cooler, in order to reach to selfcleaning condition for the compressor.

In Chapter 6, the PF scheme was extended to the general class of nonlinear/nonGaussian dynamical systems, with noisy inputs. Noise structures in the measurement signals and the state evolution model were statistically unknown, and the time-steps had un-even length. To evaluate the performance of the developed framework for multivariate systems, the well-known UNGM was extended to complex space as a BNGM with nonuniform time-steps. Instead of statistical parameters, the noise structure of BNGM was represented by sample sets, drawn from arbitrary joint distributions. The simulation results showed a superior performance when RPF scheme was employed, compared to the performance of APF, i.e., root mean squares error for RPF was smaller than APF by $17 \%$ for UNGM and $30 \%$ for BNGM.

In the next step, to examine the performance of the developed framework on experimental data, three-year operating data of a GTE were utilized. At first, a thermodynamic model of the gas-path and an empirical model for the fuel valve were developed to serve as the GTE measurement model. The degradation symptoms of the turbine were assumed the state of the system, and the measurable input and output of the system were attributed to the state. To predict the evolving state for the next time-step, a novel time-based model was introduced to predict performance deterioration of the turbine, where the system input, i.e. the GTE power was taken into account. Since the measurement noise was statistically unknown, a methodology was proposed to estimate the likelihood for updating the particle weights in the recursive state estimation. With regards to the background knowledge on the turbine degradation symptoms, state estimation results were superior with the framework using RPF compared to APF. The framework showed promising performance in simultaneous estimation of the symptoms under noisy measurement of the input / output.

\subsection{Recommendations for Future Work}

In addition to the work covered in this thesis, there are some areas to be covered by 
future research to enhance the achievements and to expand the acquired knowledge on the overall objectives of this thesis.

- $\quad$ The physics-based models developed and enhanced in Chapters 2 and 3 were based on single shaft GTE configuration, however the approach is not limited to this case and the developed performance indicators can similarly be used for two shaft and three shaft GTEs.

- The physics-based modeling approach showed promising result in calculating baseline values for anomaly detection. In many GTEs, more parameters such as compressor discharge pressure and temperature are measured by the operating system. The same physics-based approach can be used to predict the baselines for such parameters and respectively define performance indicators to evaluate GTE performance.

- Effects of available ambient and operating conditions on short-term performance deterioration of the GTE were studied in Chapter 5. There are more factors influencing on the fouling phenomenon such as the type and the size of the particulate matter. Upon availability of atmospheric pollution data, compressor fouling can more extensively be investigated with respect to every effective factor.

- Apart from GTEs, compressors are used in various industries. Fouling in a compressor has direct effects on its performance map. Deviation of the compressor map from the clean condition can be studied to establish time-based functions to predict the symptoms of performance deterioration in individual compressors.

- $\quad$ The developed techniques in Chapters 4 and 6 can estimate the turbine degradation symptoms using GTE measurements. A further step can be taken from estimation of the symptoms to identification of the fault modes, e.g. tip clearance and surface roughness increase.

- $\quad$ To evaluate robustness of the proposed degradation model in Chapter 6 to missing of training data, an empirical study to be performed and sensitivity of the model to be investigated.

- The developed state estimation framework in Chapter 6 to be applied and examined for other NSI systems and applications, such as degradation state estimation in gearboxes and batteries, and simultaneous localization and mapping (SLAM). 


\section{Bibliography}

[1] Y. Li and P. Nilkitsaranont, "Gas turbine performance prognostic for condition-based maintenance," Appl. Energy, vol. 86, pp. 2152-2161, 2009.

[2] A. R. Patnaik, V. Narasimhan, A. Nayak and P. C. Patnaik, "An integrated PHM approach for gas turbine engines," in Electrical and Computer Engineering, 2006. CCECE'06. Canadian Conference On, 2006, pp. 2476-2479.

[3] C. B. Meher-Homji, M. Chaker and A. F. Bromley, "The fouling of axial flow compressors: Causes, effects, susceptibility, and sensitivity," in ASME Turbo Expo 2009: Power for Land, Sea, and Air, 2009, pp. 571-590.

[4] R. Kurz and K. Brun, "Degradation of gas turbine performance in natural gas service," Journal of Natural Gas Science and Engineering, vol. 1, pp. 95-102, 2009.

[5] A. K. Jardine, D. Lin and D. Banjevic, "A review on machinery diagnostics and prognostics implementing condition-based maintenance," Mechanical Systems and Signal Processing, vol. 20, pp. 1483-1510, 2006.

[6] R. Ganguli, Gas Turbine Diagnostics: Signal Processing and Fault Isolation. CRC press, 2012.

[7] A. Von Flotow, Apparatus and Method for Predicting Failures of Spinning Disks in Turbo-Machinery, 2003.

[8] M. Neumann, F. Dreier, P. Günther, A. Fischer, J. Czarske, F. Holzinger and H. Schiffer, "A laser-optical sensor system for simultaneous tip clearance and vibration monitoring of compressor rotor blades," in ASME Turbo Expo 2014: Turbine Technical Conference and Exposition, 2014, pp. V006T06A002-V006T06A002.

[9] P. P. Walsh and P. Fletcher, Gas Turbine Performance. Malden, USA: Blackwell Science Ltd., 2004.

[10] I. Boxx, C. Slabaugh, P. Kutne, R. Lucht and W. Meier, "3kHz PIV/OH-PLIF measurements in a gas turbine combustor at elevated pressure," Proceedings of the Combustion Institute, vol. 35, pp. 3793-3802, 2015.

[11] Y. Li, "Performance-analysis-based gas turbine diagnostics: A review," Proc. Inst. Mech. Eng. A: J. Power Energy, vol. 216, pp. 363-377, 2002. 
[12] M. H. Schoeller, R. Orsagh, M. Roemer and L. C. Angello, "Sensor validation and recovery for combustion turbines in electrical power generation," in ASME Turbo Expo 2004: Power for Land, Sea, and Air, 2004, pp. 71-80.

[13] I. Loboda, Gas Turbine Diagnostics. INTECH Open Access Publisher, 2012.

[14] S. G. Barad, P. Ramaiah, R. Giridhar and G. Krishnaiah, "Neural network approach for a combined performance and mechanical health monitoring of a gas turbine engine," Mechanical Systems and Signal Processing, vol. 27, pp. 729-742, 2012.

[15] M. Shevchenko, S. Yepifanov and I. Loboda, "Ridge estimation and principal component analysis to solve an ill-conditioned problem of estimating unmeasured gas turbine parameters," in ASME Turbo Expo 2013: Turbine Technical Conference and Exposition, 2013, pp. V004T06A008-V004T06A008.

[16] I. K. Fodor, "A survey of dimension reduction techniques," Technical Report UCRL-ID-148494, Lawrence Livermore National Laboratory, 2002.

[17] Z. Yang, B. W. Ling and C. Bingham, "Trend extraction based on separations of consecutive empirical mode decomposition components in Hilbert marginal spectrum," Measurement, vol. 46, pp. 2481-2491, 2013.

[18] H. Hanachi, J. Liu, A. Banerjee, A. Koul, M. Liang and E. Alavi, "Bladed disc crack diagnostics using blade passage signals," Measurement Science and Technology, vol. 23, 2012.

[19] J. Yan and J. Lee, "Degradation assessment and fault modes classification using logistic regression," Journal of Manufacturing Science and Engineering, vol. 127, pp. 912-914, 2005.

[20] D. J. Bryg, G. Mink and L. C. Jaw, "Combining lead functions and logistic regression for predicting failures on an aircraft engine," in ASME Turbo Expo 2008: Power for Land, Sea, and Air, 2008, pp. 19-26.

[21] M. Venturini and D. Therkorn, "Application of a Statistical Methodology for Gas Turbine Degradation Prognostics to Alstom Field Data," Journal of Engineering for Gas Turbines and Power, vol. 135, pp. 091603, 2013.

[22] B. Sinha, "Trend prediction from steam turbine responses of vibration and eccentricity," Proc. Inst. Mech. Eng. A: J. Power Energy, vol. 216, pp. 97-103, 2002.

[23] G. Aker and H. Saravanamuttoo, "Predicting gas turbine performance degradation due to compressor fouling using computer simulation techniques," Journal of Engineering for Gas Turbines and Power, vol. 111, pp. 343-350, 1989. 
[24] M. Odabaee, M. M. Shanechi and K. Hooman, "CFD simulation and FE analysis of a high pressure ratio radial inflow turbine," in 19AFMC: 19th Australasian Fluid Mechanics Conference, 2014, pp. 1-4.

[25] H. Hanachi, J. Liu, A. Banerjee, Y. Chen and A. Koul, "A physics-based performance indicator for gas turbine engines under variable operating conditions," in ASME Turbo Expo 2014: Turbine Technical Conference and Exposition, 2014, pp. V006T06A021-V006T06A021.

[26] H. Hanachi, J. Liu, A. Banerjee, Y. Chen and A. Koul, "A Physics-Based Modeling Approach for Performance Monitoring in Gas Turbine Engines," Transactions on Reliability, vol. PP, 2014.

[27] V. Venkatasubramanian, R. Rengaswamy, K. Yin and S. N. Kavuri, "A review of process fault detection and diagnosis: Part I: Quantitative model-based methods," Comput. Chem. Eng., vol. 27, pp. 293-311, 2003.

[28] A. Feldman, H. V. de Castro, A. van Gemund and G. Provan, "Model-based diagnostic decision-support system for satellites," in Aerospace Conference, 2013 IEEE, 2013, pp. 1-14.

[29] S. M. Namburu, M. Wilcutts, S. Chigusa, L. Qiao, K. Choi and K. Pattipati, "Systematic data-driven approach to real-time fault detection and diagnosis in automotive engines," in Autotestcon, 2006 IEEE, 2006, pp. 59-65.

[30] J. Luo, M. Namburu, K. R. Pattipati, L. Qiao and S. Chigusa, "Integrated modelbased and data-driven diagnosis of automotive antilock braking systems," Systems, Man and Cybernetics, Part A: Systems and Humans, IEEE Transactions On, vol. 40, pp. 321$336,2010$.

[31] L. Yu, D. Cleary, M. Osborn and V. Rajiv, "Information fusion strategy for aircraft engine health management," in ASME Turbo Expo 2007: Power for Land, Sea, and Air, 2007, pp. 531-538.

[32] J. Liu, W. Wang, F. Ma, Y. Yang and C. Yang, "A data-model-fusion prognostic framework for dynamic system state forecasting," Eng Appl Artif Intell, vol. 25, pp. 814$823,2012$.

[33] Y. Wang, Y. Dong, J. Qiao and L. Ma, "A two-stage model of state prognosis based on vibration monitoring information," in Applied Mechanics and Materials, 2010, pp. 2182-2188.

[34] M. Pecht and R. Jaai, "A prognostics and health management roadmap for information and electronics-rich systems," Microelectronics Reliability, vol. 50, pp. 317$323,2010$. 
[35] R. Duda, P. Hart and D. Stork, Pattern Classification. Danvers, MA, USA, WileyInterscience, 2001.

[36] J. V. John, N. V. Nirmalan, J. C. Intile, S. W. Szepek, D. J. Peczka and B. S. Carey, Systems, Methods, and Apparatus for Detecting Failure in Gas Turbine Hardware, 2014.

[37] J. E. Hershey, B. E. Osborn, D. L. Gardner, R. J. Ruiz and W. L. Herron, Aircraft Gas Turbine Engine Blade Tip Clearance Control, 2012.

[38] I. Loboda and S. Yepifanov, "On the selection of an optimal pattern recognition technique for gas turbine diagnosis," in ASME Turbo Expo 2013: Turbine Technical Conference and Exposition, 2013, pp. V004T06A019-V004T06A019.

[39] S. Kumano, N. Mikami and K. Aoyama, "Advanced gas turbine diagnostics using pattern recognition," in ASME 2011 Turbo Expo: Turbine Technical Conference and Exposition, 2011, pp. 179-187.

[40] J. Schürmann, Pattern Classification: A Unified View of Statistical and Neural Approaches. Wiley Online Library, 1996.

[41] P. Baraldi, F. Di Maio, D. Genini and E. Zio, "Reconstruction of missing data in multidimensional time series by fuzzy similarity," Applied Soft Computing, vol. 26, pp. $1-9,2015$.

[42] F. Z. Ghavidel, J. Claesen, T. Burzykowski and D. Valkenborg, "Comparison of the mahalanobis distance and pearson's $\chi^{2}$ statistic as measures of similarity of isotope patterns," J. Am. Soc. Mass Spectrom., vol. 25, pp. 293-296, 2014.

[43] V. Perduca and G. Nuel, "Measuring the Influence of Observations in HMMs Through the Kullback-Leibler Distance," Signal Processing Letters, IEEE, vol. 20, pp. 145-148, 2013.

[44] W. Lei, "Semi-supervised spectral clustering combined with bayesian decision," in Computational Intelligence and Design (ISCID), 2014 Seventh International Symposium On, 2014, pp. 437-440.

[45] C. Cortes and V. Vapnik, "Support-vector networks," Mach. Learning, vol. 20, pp. 273-297, 1995.

[46] B. Yang and A. Widodo, "Support vector machine for machine fault diagnosis and prognosis," Journal of System Design and Dynamics, vol. 2, pp. 12-23, 2008.

[47] D. Zhou, H. Zhang and S. Weng, "A New Gas Path Fault Diagnostic Method of Gas Turbine Based on Support Vector Machine," Journal of Engineering for Gas Turbines and Power, vol. 137, pp. 102605, 2015. 
[48] W. Hu, J. Liu, J. Cui, Y. Gao, B. Cui and L. Jiang, "Fault diagnosis of gas turbine based on support vector machine," in Control and Decision Conference (2014 CCDC), the 26th Chinese, 2014, pp. 2853-2856.

[49] Z. Wang, N. Zhao, W. Wang, R. Tang and S. Li, "A Fault Diagnosis Approach for Gas Turbine Exhaust Gas Temperature Based on Fuzzy C-Means Clustering and Support Vector Machine," Mathematical Problems in Engineering, 2014.

[50] W. Zhao, C. Hou and Q. Wang, "Diagnosis of aircraft engine performance deterioration based on support vector machines," in Reliability, Maintainability and Safety (ICRMS), 2014 International Conference On, 2014, pp. 44-48.

[51] X. WEI, C. LU, C. Wang, J. LU and Y. LI, "Applications of Support Vector Machines to Aeroengine Fault Diagnosis [J]," Journal of Aerospace Power, vol. 6, pp. 020, 2004 .

[52] H. Ying, J. SUN, G. YANG and B. Jie, "The application of support vector machines to gas turbine performance diagnosis," Chinese Journal of Aeronautics, vol. 18, pp. 1519, 2005.

[53] K. Medjaher, D. A. Tobon-Mejia and N. Zerhouni, "Remaining useful life estimation of critical components with application to bearings," Reliability, IEEE Transactions On, vol. 61, pp. 292-302, 2012.

[54] S. S. Tayarani-Bathaie, Z. N. S. Vanini and K. Khorasani, "Dynamic neural network-based fault diagnosis of gas turbine engines." Neurocomputing, vol. 125, pp. 153-165, 2014.

[55] I. Loboda and Y. Feldshteyn, "Polynomials and Neural Networks for Gas Turbine Monitoring: a Comparative Study," International Journal of Turbo and Jet Engines, vol. 28, pp. 227-236, 2011.

[56] U. Campora, M. Capelli, C. Cravero and R. Zaccone, "Metamodels of a gas turbine powered marine propulsion system for simulation and diagnostic purposes," Journal of Naval Architecture and Marine Engineering, vol. 12, pp. 1-14, 2015.

[57] I. Loboda, S. Yepifanov and Y. Feldshteyn, "A more realistic scheme of deviation error representation for gas turbine diagnostics," Int.J.Turbo Jet-Engines, vol. 30, pp. 179-189, 2013.

[58] R. Barbosa and S. Ferreira, "Industrial gas turbine diagnostics using fuzzy logic," in ASME Turbo Expo 2012: Turbine Technical Conference and Exposition, 2012, pp. 803808. 
[59] X. Zhao, Y. Liu and X. He, "Fault diagnosis of gas turbine based on fuzzy matrix and the principle of maximum membership degree," Energy Procedia, vol. 16, pp. 14481454, 2012.

[60] K. E. Holbert and K. Lin, "Nuclear power plant instrumentation fault detection using fuzzy logic," Science and Technology of Nuclear Installations, vol. 2012, 2012.

[61] M. Shabanian and M. Montazeri, "A neuro-fuzzy online fault detection and diagnosis algorithm for nonlinear and dynamic systems," International Journal of Control, Automation and Systems, vol. 9, pp. 665-670, 2011.

[62] Ö Uluyol, K. Kim and E. O. Nwadiogbu, "Synergistic use of soft computing technologies for fault detection in gas turbine engines," Systems, Man, and Cybernetics, Part C: Applications and Reviews, IEEE Transactions On, vol. 36, pp. 476-484, 2006.

[63] D. Ruan and E. E. Kerre, Fuzzy if-then Rules in Computational Intelligence: Theory and Applications. Springer Science \& Business Media, 2012.

[64] D. Subbaram Naidu, "Soft computing and intelligent systems design: theory, tools and applications, FO Karry and C. De Silva, Pearson, Addison-Wesley, New York, NY, 2004," International Journal of Robust and Nonlinear Control, vol. 16, pp. 548-551, 2006.

[65] L. Kuncheva, "How good are fuzzy if-then classifiers?" Systems, Man, and Cybernetics, Part B: Cybernetics, IEEE Transactions On, vol. 30, pp. 501-509, 2000.

[66] L. Barelli, G. Bidini and F. Bonucci, "Diagnosis of a turbocharging system of 1MW internal combustion engine," Energy Conversion and Management, vol. 68, pp. 28-39, 2013.

[67] A. Salar, A. K. Sedigh, S. Hosseini and H. Khaledi, "A hybrid EKF-fuzzy approach to fault detection and isolation of industrial gas turbines," in ASME 2011 Turbo Expo: Turbine Technical Conference and Exposition, 2011, pp. 251-260.

[68] D. Bordoloi and R. Tiwari, "Health monitoring of gear elements based on timefrequency vibration by support vector machine algorithms," in ASME 2013 Gas Turbine India Conference, 2013, pp. V001T05A019-V001T05A019.

[69] R. Precup, P. Angelov, B. S. J. Costa and M. Sayed-Mouchaweh, "An overview on fault diagnosis and nature-inspired optimal control of industrial process applications," Comput. Ind., 2015.

[70] J. Jang, "ANFIS: adaptive-network-based fuzzy inference system," Systems, Man and Cybernetics, IEEE Transactions On, vol. 23, pp. 665-685, 1993. 
[71] A. Abraham, "Adaptation of fuzzy inference system using neural learning," in Fuzzy Systems EngineeringAnonymous Springer, 2005, pp. 53-83.

[72] R. Bettocchi, M. Pinelli, P. Spina and M. Venturini, "Artificial Intelligence for the Diagnostics of Gas Turbines-Part II: Neuro-Fuzzy Approach," Journal of Engineering for Gas Turbines and Power, vol. 129, pp. 720-729, 2007.

[73] H. A. Nozari, M. A. Shoorehdeli, S. Simani and H. D. Banadaki, "Model-based robust fault detection and isolation of an industrial gas turbine prototype using soft computing techniques," Neurocomputing, vol. 91, pp. 29-47, 2012.

[74] D. M. Ghiocel and J. Altmann, A Hybrid Stochastic-Neuro-Fuzzy Model-Based System for in-Flight Gas Turbine Engine Diagnostics, 2001.

[75] D. M. Ghiocel and J. Altmann, "In-Flight Engine Diagnostics and Prognostics Using A Stochastic-Neuro-Fuzzy Inference System," 2002.

[76] M. Shabanian and M. Montazeri, "A neuro-fuzzy online fault detection and diagnosis algorithm for nonlinear and dynamic systems," International Journal of Control, Automation and Systems, vol. 9, pp. 665-670, 2011.

[77] J. Lee, F. Wu, W. Zhao, M. Ghaffari, L. Liao and D. Siegel, "Prognostics and health management design for rotary machinery systems-Reviews, methodology and applications," Mechanical Systems and Signal Processing, vol. 42, pp. 314-334, 2014.

[78] J. Sun, H. Zuo, W. Wang and M. G. Pecht, "Application of a state space modeling technique to system prognostics based on a health index for condition-based maintenance," Mechanical Systems and Signal Processing, vol. 28, pp. 585-596, 2012.

[79] I. Loboda and S. Yepifanov, "A mixed data-driven and model based fault classification for gas turbine diagnosis," in ASME Turbo Expo 2010: Power for Land, Sea, and Air, 2010, pp. 257-265.

[80] A. Staroselsky and T. J. Martin, "Creep, plasticity and fatigue of single crystal superalloys: Physics-based life prediction for turbine components in severe operating environments," in Inelastic Behavior of Materials and Structures Under Monotonic and Cyclic LoadingAnonymous Springer, 2015, pp. 207-241.

[81] E. Tsoutsanis, N. Meskin, M. Benammar and K. Khorasani, "A component map tuning method for performance prediction and diagnostics of gas turbine compressors," Appl. Energy, vol. 135, pp. 572-585, 2014.

[82] D. Zhou, H. Zhang and S. Weng, "A novel prognostic model of performance degradation trend for power machinery maintenance," Energy, vol. 78, pp. 740-746, 2014. 
[83] M. A. Ghafir, Y. Li and L. Wang, "Creep Life Prediction for Aero Gas Turbine Hot Section Component Using Artificial Neural Networks," Journal of Engineering for Gas Turbines and Power, vol. 136, pp. 031504, 2014.

[84] R. E. Kalman, "A new approach to linear filtering and prediction problems," Journal of Fluids Engineering, vol. 82, pp. 35-45, 1960.

[85] A. H. Jazwinski, "Stochastic processes and filtering theory," 1970.

[86] S. J. Julier and J. K. Uhlmann, "New extension of the kalman filter to nonlinear systems," in AeroSense'97, 1997, pp. 182-193.

[87] A. J. Volponi, H. DePold, R. Ganguli and C. Daguang, "The use of Kalman filter and neural network methodologies in gas turbine performance diagnostics: a comparative study," Journal of Engineering for Gas Turbines and Power, vol. 125, pp. 917-924, 2003.

[88] S. Borguet and O. Léonard, "Coupling principal component analysis and Kalman filtering algorithms for on-line aircraft engine diagnostics," Control Eng. Pract., vol. 17, pp. 494-502, 2009.

[89] N. J. Gordon, D. J. Salmond and A. F. Smith, "Novel approach to nonlinear/nongaussian bayesian state estimation," in IEE Proceedings F (Radar and Signal Processing), 1993, pp. 107-113.

[90] J. Liu, W. Wang and F. Ma, "A regularized auxiliary particle filtering approach for system state estimation and battery life prediction," Smart Mater Struct, vol. 20, pp. 075021, 2011.

[91] C. Musso, N. Oudjane and F. Le Gland, "Improving regularised particle filters," in Sequential Monte Carlo Methods in PracticeAnonymous Springer, 2001, pp. 247-271.

[92] M. K. Pitt and N. Shephard, "Filtering via simulation: Auxiliary particle filters," Journal of the American Statistical Association, vol. 94, pp. 590-599, 1999.

[93] N. Daroogheh, N. Meskin and K. Khorasani, "A novel particle filter parameter prediction scheme for failure prognosis," in American Control Conference (ACC), 2014, 2014, pp. 1735-1742.

[94] F. Lu, J. Huang and Y. Lv, "Gas path health monitoring for a turbofan engine based on a nonlinear filtering approach," Energies, vol. 6, pp. 492-513, 2013.

[95] J. Ching, J. L. Beck and K. A. Porter, "Bayesian state and parameter estimation of uncertain dynamical systems," Prob. Eng. Mech., vol. 21, pp. 81-96, 2006. 
[96] T. B. Schön, A. Wills and B. Ninness, "System identification of nonlinear statespace models," Automatica, vol. 47, pp. 39-49, 2011.

[97] H. Hanachi, J. Liu, A. Banerjee and Y. Chen, "A framework with nonlinear system model and nonparametric noise for gas turbine degradation state estimation," Measurement Science and Technology, vol. 26, 065604, 2015.

[98] M. S. Kan, A. C. Tan and J. Mathew, "A review on prognostic techniques for nonstationary and non-linear rotating systems," Mechanical Systems and Signal Processing, vol. 62, pp. 1-20, 2015.

[99] D. Subbaram Naidu, "Soft computing and intelligent systems design: theory, tools and applications, FO Karry and C. De Silva, Pearson, Addison-Wesley, New York, NY, 2004," International Journal of Robust and Nonlinear Control, vol. 16, pp. 548-551, 2006.

[100] R. J. Patton, P. M. Frank and R. N. Clark, Issues of Fault Diagnosis for Dynamic Systems. Springer Science \& Business Media, 2013.

[101] K. L. Tsui, N. Chen, Q. Zhou, Y. Hai and W. Wang, "Prognostics and Health Management: A Review on Data Driven Approaches," Mathematical Problems in Engineering, vol. 2015, 2015.

[102] A. Khaleghei and V. Makis, "Model parameter estimation and residual life prediction for a partially observable failing system," Naval Research Logistics (NRL), vol. 62, pp. 190-205, 2015.

[103] N. Puggina and M. Venturini, "Development of a statistical methodology for gas turbine prognostics," Journal of Engineering for Gas Turbines and Power, vol. 134, pp. $022401,2012$.

[104] H. Wang and Y. Jiang, "Performance reliability prediction of complex system based on the condition monitoring information," Mathematical Problems in Engineering, vol. 2013, 2013.

[105] J. Sun, H. Zuo, W. Wang and M. G. Pecht, "Application of a state space modeling technique to system prognostics based on a health index for condition-based maintenance," Mechanical Systems and Signal Processing, vol. 28, pp. 585-96, 04, 2012.

[106] G. Kacprzynski, A. Sarlashkar, M. Roemer, A. Hess and B. Hardman, "Predicting remaining life by fusing the physics of failure modeling with diagnostics," JOM, vol. 56, pp. 29-35, 2004.

[107] C. J. Li and H. Lee, "Gear fatigue crack prognosis using embedded model, gear dynamic model and fracture mechanics," Mechanical Systems and Signal Processing, vol. 19, pp. 836-846, 2005. 
[108] J. Coble and J. W. Hines, "Identifying Suitable Degradation Parameters for Individual-Based Prognostics," Diagnostics and Prognostics of Engineering Systems: Methods and Techniques: Methods and Techniques, pp. 135, 2012.

[109] G. Harrison, "Translation of service usage into component life consumption," Recommended Practices for Monitoring Gas Turbine Engine Life Consumption", RTO Technical Report, vol. 28, 2000.

[110] A. Koul, Method and System for Real-Time Prognosis Analysis and Usage Based Residual Life Assessment of Turbine Engine Components and Display, 2012.

[111] T. J. Carter, "Common failures in gas turbine blades," Eng. Failure Anal., vol. 12, pp. 237-247, 2005.

[112] L. Feng, H. Wang, X. Si and H. Zou, "A state-space-based prognostic model for hidden and age-dependent nonlinear degradation process," Automation Science and Engineering, IEEE Transactions On, vol. 10, pp. 1072-1086, 2013.

[113] X. Si, W. Wang, C. Hu and D. Zhou, "Remaining useful life estimation-A review on the statistical data driven approaches," Eur. J. Oper. Res., vol. 213, pp. 1-14, 2011.

[114] Y. Peng, M. Dong and M. J. Zuo, "Current status of machine prognostics in condition-based maintenance: a review," The International Journal of Advanced Manufacturing Technology, vol. 50, pp. 297-313, 2010.

[115] C. Amann and K. Kadau, Method for Estimating Crack Length Progressions, 2014.

[116] H. Hanachi, E. Alavi, J. Liu, A. Banerjee, A. Koul and M. Liang, "Bladed disk crack detection through advanced analysis of blade time of arrival signal," in Prognostics and Health Management (PHM), 2012 IEEE Conference On, 2012, pp. 1-4.

[117] M. Dong, "A novel approach to equipment health management based on autoregressive hidden semi-Markov model (AR-HSMM)," Science in China Series F: Information Sciences, vol. 51, pp. 1291-1304, 2008.

[118] H. Tong, Threshold Models in Non-Linear Time Series Analysis. Springer Science \& Business Media, 2012.

[119] O. Kisi, "Pan evaporation modeling using least square support vector machine, multivariate adaptive regression splines and M5 model tree," Journal of Hydrology, 2015.

[120] J. Kasten, F. Lazeyras and D. Van De Ville, "Data-driven MRSI spectral localization via low-rank component analysis," Medical Imaging, IEEE Transactions On, vol. 32, pp. 1853-1863, 2013. 
[121] A. Rai and A. Kohli, "Simulation and analysis of nonlinear system identification using the LMS volterra filter," in Advanced Materials Research, 2012, pp. 3528-3537.

[122] Z. Liu, Y. Hu and W. Wu, "Pipe performance analysis with nonparametric regression," in SPIE Smart Structures and Materials Nondestructive Evaluation and Health Monitoring, 2011, pp. 798337-798337-9.

[123] R. Yaffee and M. McGee, "Introduction to Time Series Analysis and Forecasting with Applications of SAS and SPSS. San Diego," Cal: Academic Press, 2000.

[124] J. Liu, A. Saxena, K. Goebel, B. Saha and W. Wang, An Adaptive Recurrent Neural Network for Remaining Useful Life Prediction of Lithium-Ion Batteries, 2010.

[125] Z. Tian, L. Wong and N. Safaei, "A neural network approach for remaining useful life prediction utilizing both failure and suspension histories," Mechanical Systems and Signal Processing, vol. 24, pp. 1542-1555, 2010.

[126] Z. Tian, "An artificial neural network method for remaining useful life prediction of equipment subject to condition monitoring," J. Intell. Manuf., vol. 23, pp. 227-237, 2012.

[127] A. K. Mahamad, S. Saon and T. Hiyama, "Predicting remaining useful life of rotating machinery based artificial neural network," Comput. Math. Appl., vol. 60, pp. 1078-1087, 2010.

[128] L. A. Y. Alrabady, "An online-integrated condition monitoring and prognostics framework for rotating equipment," 2014.

[129] J. R. Jang, C. Sun and E. Mizutani, "Neuro-fuzzy and soft computing; a computational approach to learning and machine intelligence," 1997.

[130] M. El-Koujok, R. Gouriveau and N. Zerhouni, "Reducing arbitrary choices in model building for prognostics: An approach by applying parsimony principle on an evolving neuro-fuzzy system," Microelectronics Reliability, vol. 51, pp. 310-320, 2011.

[131] W. Wang, "An adaptive predictor for dynamic system forecasting," Mechanical Systems and Signal Processing, vol. 21, pp. 809-823, 2007.

[132] W. Q. Wang, M. F. Golnaraghi and F. Ismail, "Prognosis of machine health condition using neuro-fuzzy systems," Mechanical Systems and Signal Processing, vol. 18, pp. 813-831, 2004.

[133] H. I. H. Saravanamuttoo, Gas Turbine Theory. Upper Saddle River, N.J: Pearson Prentice Hall, 2009.

[134] M. P. Boyce, Gas Turbine Engineering Handbook. Butterworth-Heinemann, 2011. 
[135] R. Sonntag and G. Van Wylen, "Introduction to Thermodynamics, 1991," .

[136] T. A. P. S. Apparao, "Effects of the combustion and heat of combustion of hydrocarbon fuels on gas turbine cycle performance," $1968,1968$.

[137] Aeronautical Research Council (Great Britain), D. Fielding and J. Topps, Thermodynamic Data for the Calculation of Gas Turbine Performance. HM Stationery Office, 1959.

[138] R. Kurz and K. Brun, "Degradation in gas turbine systems," Journal of Engineering for Gas Turbines and Power(Transactions of the ASME), vol. 123, pp. 7077, 2001.

[139] C. S. Byington, M. J. Roemer and M. J. Watson, Prognostic Enhancements to Diagnostic Systems (PEDS) Applied to Shipboard Power Generation Systems, 2004.

[140] M. P. Boyce and F. Gonzalez, "A study of on-line and off-line turbine washing to optimize the operation of a gas turbine," Journal of Engineering for Gas Turbines and Power, vol. 129, pp. 114-122, 2007.

[141] C. B. Meher-Homji and A. Bromley, "Gas turbine axial compressor fouling and washing," in Proceedings of the 33rd Turbomachinery Symposium, 2004, pp. 20-23.

[142] C. B. Meher-Homji, M. Chaker and H. Motiwalla, "Gas turbine performance deterioration," in Proceedings of the 30th Turbomachinery Symposium, 2001, pp. 17-20.

[143] G. Aker and H. Saravanamuttoo, "Predicting gas turbine performance degradation due to compressor fouling using computer simulation techniques," Journal of Engineering for Gas Turbines and Power, vol. 111, pp. 343-350, 1989.

[144] R. J. Hyndman and A. B. Koehler, "Another look at measures of forecast accuracy," Int. J. Forecast., vol. 22, pp. 679-688, 2006.

[145] M. Pinelli and P. Spina, "Gas turbine field performance determination: sources of uncertainties," Journal of Engineering for Gas Turbines and Power, vol. 124, pp. 155$160,2002$.

[146] K. Mathioudakis and T. Tsalavoutas, "Uncertainty reduction in gas turbine performance diagnostics by accounting for humidity effects," Journal of Engineering for Gas Turbines and Power, vol. 124, pp. 801-808, 2002.

[147] B. G. Kyle, Chemical and Process Thermodynamics. New Jersey: Englewood Cliffs Prentice Hall, 1984.

[148] Y. A. Cengel, M. A. Boles and M. Kanoğlu, Thermodynamics: An Engineering Approach. McGraw-Hill New York, 2011. 
[149] A. L. Buck, "New equations for computing vapor pressure and enhancement factor," J. Appl. Meteorol., vol. 20, pp. 1527-1532, 1981.

[150] D. J. Sheskin, Handbook of Parametric and Nonparametric Statistical Procedures. crc Press, 2003.

[151] A. Saxena, K. Goebel, D. Simon and N. Eklund, "Damage propagation modeling for aircraft engine run-to-failure simulation," in Prognostics and Health Management, 2008. PHM 2008. International Conference On, 2008, pp. 1-9.

[152] M. Sanjeev Arulampalam, S. Maskell, N. Gordon and T. Clapp, "A tutorial on particle filters for online nonlinear/non-Gaussian Bayesian tracking," Signal Processing, IEEE Transactions On, vol. 50, pp. 174-188, 2002.

[153] A. Doucet, N. De Freitas and N. Gordon, "An introduction to sequential monte carlo methods," in Sequential Monte Carlo Methods in PracticeAnonymous Springer, 2001, pp. 3-14.

[154] C. Musso, N. Oudjane and F. Le Gland, "Improving regularised particle filters," in Sequential Monte Carlo Methods in PracticeAnonymous Springer, 2001, pp. 247-271.

[155] D. Simon, Optimal State Estimation: Kalman, $H$ Infinity, and Nonlinear Approaches. John Wiley \& Sons, 2006.

[156] F. J. Brooks, "GE gas turbine performance characteristics," GE Power Systems, Schenectady, NY, 2000.

[157] J. Stalder, "Gas turbine compressor washing state of the art: Field experiences," Journal of Engineering for Gas Turbines and Power, vol. 123, pp. 363$370,2001$.

[158] H. Hanachi, J. Liu, A. Banerjee and Y. Chen, "Effects of Humidity Condensation on the Trend of Gas Turbine Performance Deterioration," Journal of Engineering for Gas Turbines and Power, 2015.

[159] G. J. Kacprzynski, M. Gumina, M. J. Roemer, D. E. Caguiat and T. R. Galie, "A prognostic modeling approach for predicting recurring maintenance for shipboard propulsion systems," in Proceedings of ASME Turbo Expo 2001, New Orleans, LA USA, 2001, .

[160] I. Loboda and Y. Feldshteyn, "Polynomials and neural networks for gas turbine monitoring: A comparative study," in ASME Turbo Expo 2010: Power for Land, Sea, and Air, 2010, pp. 417-427.

[161] K. Salahshoor, M. Kordestani and M. S. Khoshro, "Fault detection and diagnosis of an industrial steam turbine using fusion of SVM (support vector machine) and ANFIS 
(adaptive neuro-fuzzy inference system) classifiers," Energy, vol. 35, pp. 5472-5482, 2010.

[162] K. Salahshoor, M. S. Khoshro and M. Kordestani, "Fault detection and diagnosis of an industrial steam turbine using a distributed configuration of adaptive neuro-fuzzy inference systems," Simulation Modelling Practice and Theory, vol. 19, pp. 1280-1293, 2011.

[163] M. E. Cohen and D. L. Hudson, Comparative Approaches to Medical Reasoning. World Scientific, 1995.

[164] T. Takagi and M. Sugeno, "Fuzzy identification of systems and its applications to modeling and control," Systems, Man and Cybernetics, IEEE Transactions On, pp. 116$132,1985$.

[165] J. P. Bons, "A review of surface roughness effects in gas turbines," Journal of Turbomachinery, vol. 132, pp. 021004, 2010.

[166] R. Kurz, "Gas turbine performance," in Proceedings of the Thirty-Fourth Turbomachinery Symposium., Houston, TX, 2005, .

[167] R. Kurz and K. Brun, "Gas turbine tutorial—maintenance and operating practices effects on degradation and life," in 36th Turbomachinery Symposium, Houston, TX, September, 2007, pp. 10-13.

[168] A. Granovskiy, M. Kostege and N. Lomakin, "Parametrical investigation of turbine stages with open tip clearance for the purpose of stage efficiency increase," in ASME Turbo Expo 2010: Power for Land, Sea, and Air, 2010, pp. 1425-1432.

[169] L. Helmers and J. Klingmann, "Unshrouded rotor tip clearance effects in expander cycle turbines," in ASME Turbo Expo 2002: Power for Land, Sea, and Air, 2002, pp. 127-134.

[170] H. Matsuda, F. Otomo, H. Kawagishi, A. Inomata, Y. Niizeki and T. Sasaki, "Influence of surface roughness on turbine nozzle profile loss and secondary loss," in ASME Turbo Expo 2006: Power for Land, Sea, and Air, 2006, pp. 781-788.

[171] M. Montis, R. Niehuis and A. Fiala, "Aerodynamic measurements on a low pressure turbine cascade with different levels of distributed roughness," in ASME 2011 Turbo Expo: Turbine Technical Conference and Exposition, 2011, pp. 457-467.

[172] S. Won, W. W. Melek and F. Golnaraghi, "A Kalman/particle filter-based position and orientation estimation method using a position sensor/inertial measurement unit hybrid system," Industrial Electronics, IEEE Transactions On, vol. 57, pp. 1787-1798, 2010. 
[173] F. Gustafsson, "Particle filter theory and practice with positioning applications," Aerospace and Electronic Systems Magazine, IEEE, vol. 25, pp. 53-82, 2010.

[174] B. W. Silverman, Density Estimation for Statistics and Data Analysis. CRC press, 1986.

[175] H. Hanachi, J. Liu, A. Banerjee and Y. Chen, "Effects of the intake air humidity on the gas turbine performance monitoring," in ASME Turbo Expo 2015: Turbine Technical Conference and Exposition, Montreal, Canada, 2015. 


\section{Appendix}

Permissions to Reproduce Copyrighted Material 
From: M.E. Brennan <me.brennan@ieee.org>

Sent: June-30-15 12:17 PM

To: Houman Hanachi

Cc: M.E. Brennan; Frank Pepe

Subject: Fwd: Fw: My paper with IEEE transactions on Reliability, Request for permission

Dear Houman,

The IEEE does not require individuals working on a thesis to obtain a formal reuse license however, you must follow the requirements listed below:

\section{Textual Material}

Using short quotes or referring to the work within these papers) users must give full credit to the original source (author, paper, publication) followed by the IEEE copyright line $\odot$ [Year of publication] IEEE.

In the case of illustrations or tabular material, we require that the copyright line $($ ) [Year of original publication] IEEE appear prominently with each reprinted figure and/or table.

If a substantial portion of the original paper is to be used, and if you are not the senior author, also obtain the senior author's approval.

\section{Full-Text Article}

If you are using the entire IEEE copyright owned article, the following IEEE copyright/ credit notice should be placed prominently in the references: (C) [year of original publication] IEEE. Reprinted, with permission, from [author names, paper title, IEEE publication title, and month/year of publication] Only the accepted version of an IEEE copyrighted paper can be used when posting the paper or your thesis on-line. You may not use the final published version.

In placing the thesis on the author's university website, please display the following message in a prominent place on the website: In reference to IEEE copyrighted material which is used with permission in this thesis, the IEEE does not endorse any of [university/educational entity's name goes here]'s products or services. Internal or personal use of this material is permitted. If interested in reprinting/republishing IEEE copyrighted material for advertising or promotional purposes or for creating new collective works for resale or redistribution, please go to http://www.ieee.org/publications standards/publications/rights/rights link.html to learn how to obtain a License from RightsLink.

If applicable, University Microfilms and/or ProQuest Library, or the Archives of Canada may supply single copies of the dissertation.

This same information may be viewed when you request permissions using RightsLink in Xplore and choose reuse in a dissertation from the drop down menu.

Kind regards,

M.E.

Ms M.E. Brennan

IEEE

501 Hoes Lane

Piscataway, NJ 08854-4141 USA

me.brennan@ieee.org

+1 (732) 562-2660 
From: Beth Darchi <DarchiB@asme.org>

Sent: July-20-15 11:55 AM

To: Houman Hanachi

Subject: RE: Request for permission

Dear Mr. Hanachi,

It has been confirmed that your paper was presented at the GT2015 Conference, therefore, it is our pleasure to grant you permission to use all or any part of the ASME paper "Effects of the Intake Air Humidity on the Gas Turbine Performance Monitoring," by Houman Hanachi, Jie Liu, Avisekh Banerjee, Ying Chen, Paper Number GT2015-43026, as cited in your letter for inclusion in an $\mathrm{PhD}$ thesis entitled Gas turbine engine performance estimation and prediction to be published by Carleton University, Ottawa, Canada.

Permission is granted for the specific use as stated herein and does not permit further use of the materials without proper authorization. Proper attribution must be made to the author(s) of the materials. Please note: if any or all of the figures and/or Tables are of another source, permission should be granted from that outside source or include the reference of the original source. ASME does not grant permission for outside source material that may be referenced in the ASME works.

As is customary, we request that you ensure full acknowledgment of this material, the author(s), source and ASME as original publisher. Acknowledgment must be retained on all pages printed and distributed.

Many thanks for your interest in ASME publications.

Sincerely,

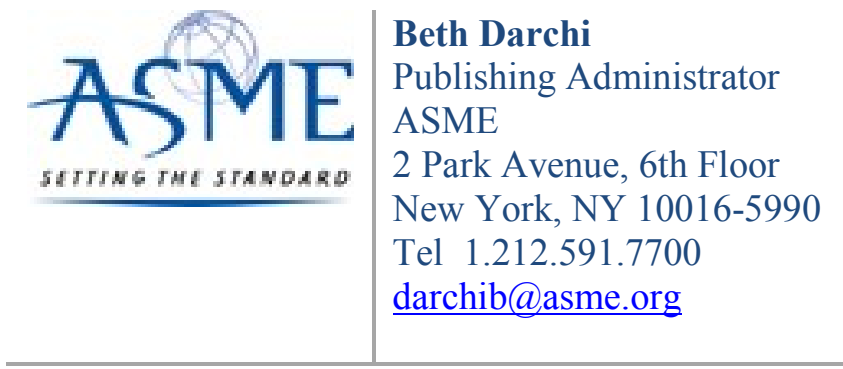


To: Houman Hanachi;

Cc: djs.birch@strath.ac.uk;

MST<mst@iop.org>;

Dear Houman Hanachi,

Thank you for your email and for taking the time to seek this permission.

When you transferred the copyright in your article to IOP, we granted back to you certain rights, including the right to include the article within any thesis or dissertation.

Please include citation details and for online use, a link to the Version of Record.

The only restriction is that if, at a later date, your thesis were to be published commercially, further permission would be required.

Please let me know if you have any further questions.

In the meantime, I wish you the best of luck with the completion of your dissertation.

Kind regards,

Zora Catterick

Publishing Assistant

IOP Publishing

Please note: We do not usually provide signed permission forms as a separate

attachment. Please print this email and provide it to your publisher as proof of permission. 
From: Beth Darchi <DarchiB@asme.org>

Sent: July-17-15 2:37 PM

To: Houman Hanachi

Subject: FW: Important: Request for permission.

Dear Mr. Hanachi, It is our pleasure to grant you permission to use all or any part of the ASME paper "Effects of Humidity Condensation on the Trend of Gas Turbine Performance Deterioration," by Houman Hanachi, Jie Liu1, Avisekh Banerjee and Ying Chen, J. Eng. Gas Turbines Power, 137(12), 2015, cited in your letter for inclusion in a $\mathrm{PhD}$ thesis to be published by Carleton University.

Permission is granted for the specific use as stated herein and does not permit further use of the materials without proper authorization. Proper attribution must be made to the author(s) of the materials. Please note: if any or all of the figures and/or Tables are of another source, permission should be granted from that outside source or include the reference of the original source. ASME does not grant permission for outside source material that may be referenced in the ASME works.

As is customary, we request that you ensure full acknowledgment of this material, the author(s), source and ASME as original publisher. Acknowledgment must be retained on all pages printed and distributed.

Many thanks for your interest in ASME publications.

Sincerely,

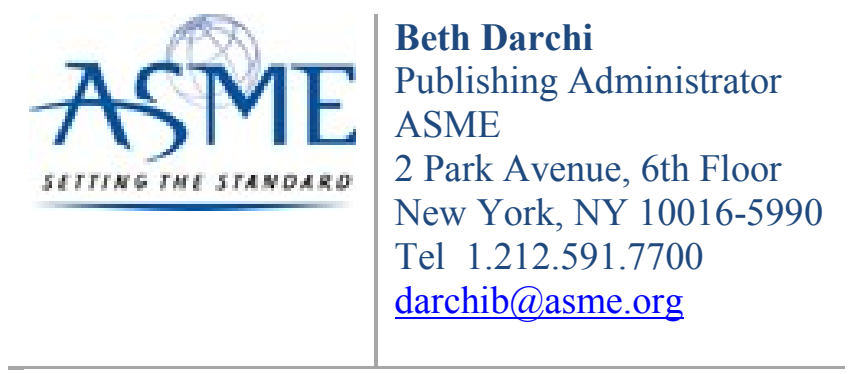

